\title{
LISTA GERAL
}

Dos

\section{BACHAREIS E DOUTORES FORMADOS}

PELA

\author{
FACULDADE DE DIREITO DE S. PAULO
}

E DOS

\section{LENTES E DIRECTORES EFFECTIVOS}

até 1900

ORGANISADA PELO

Bacharel Julio Joaquim Gonçalves Maia 


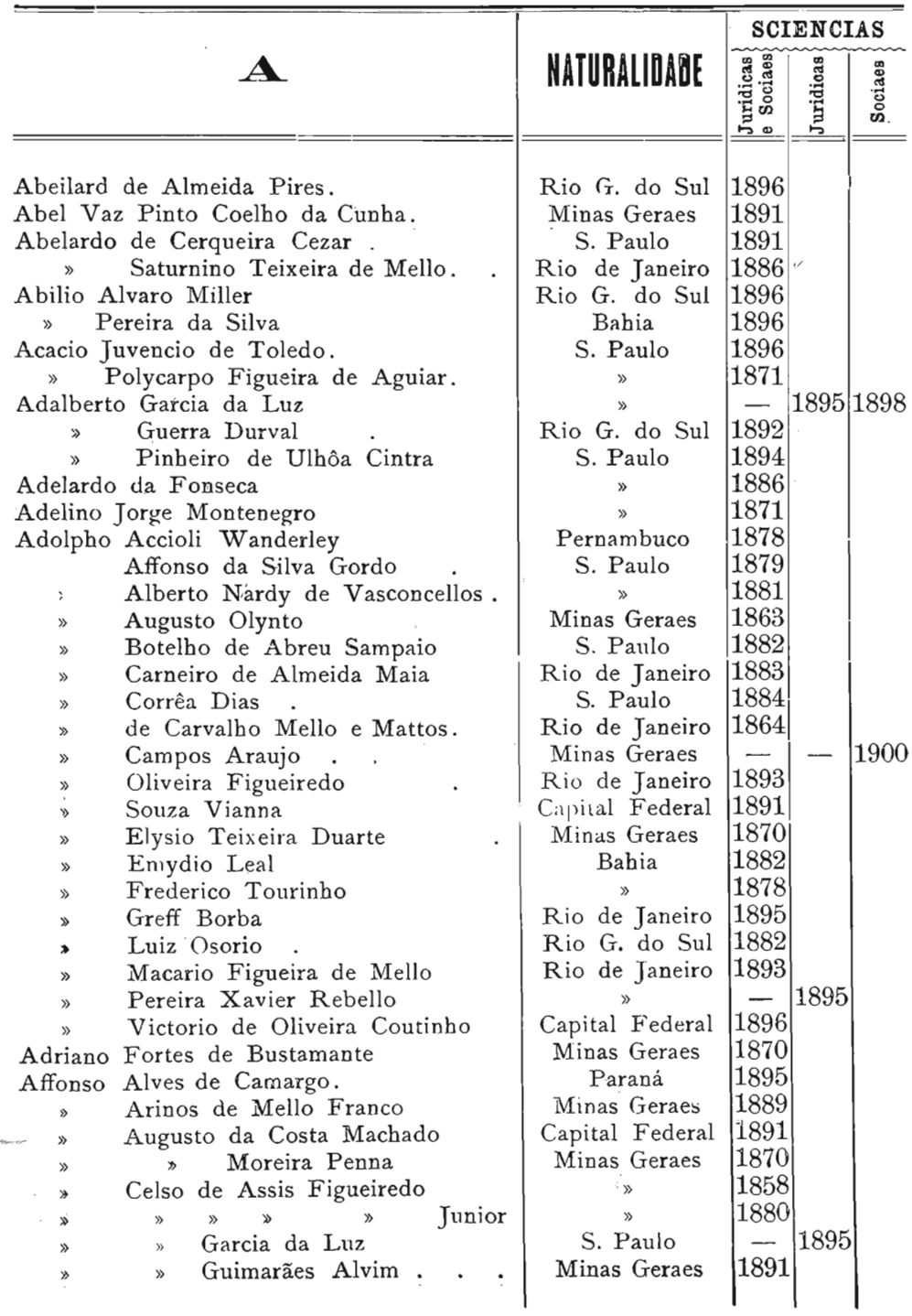




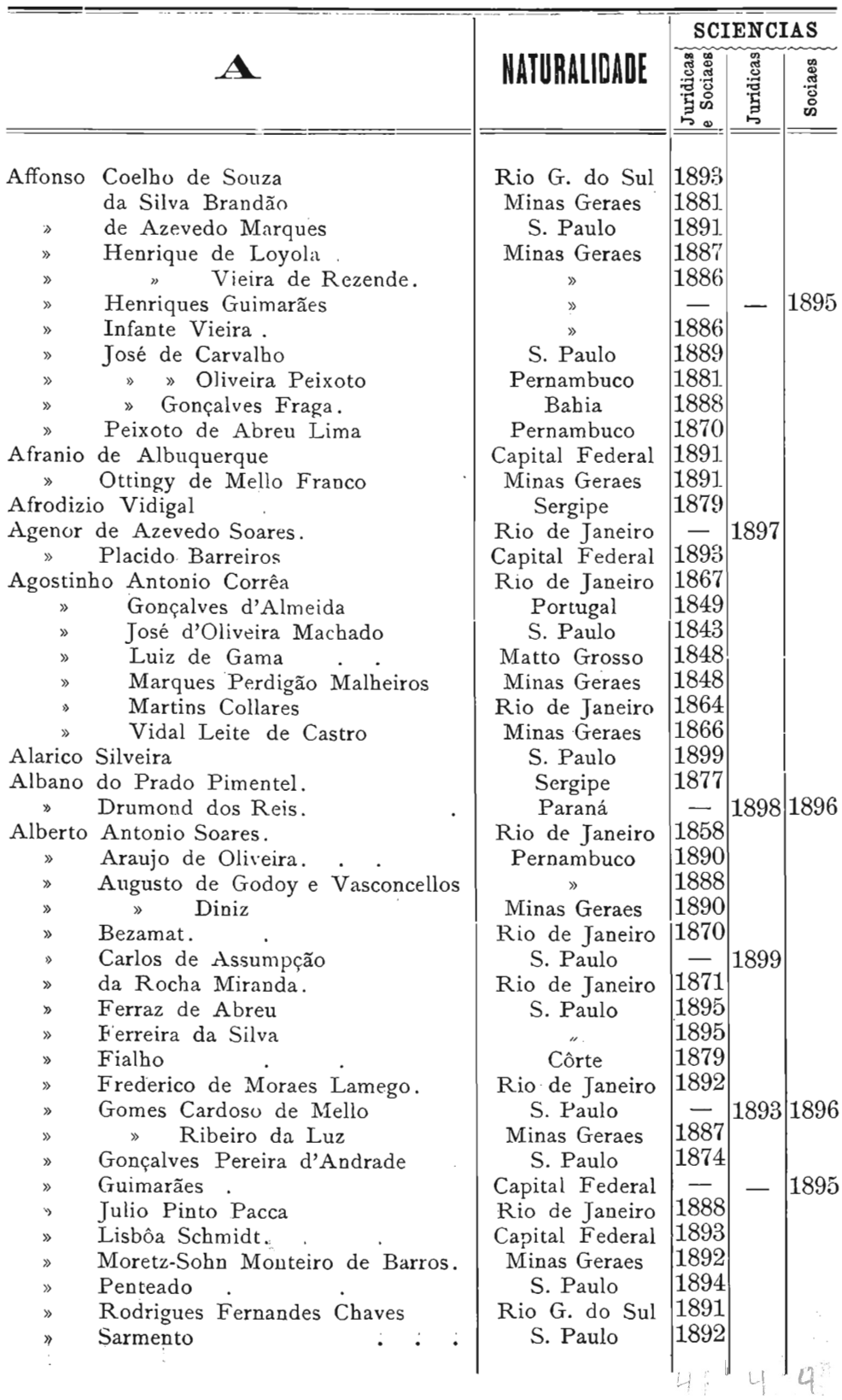




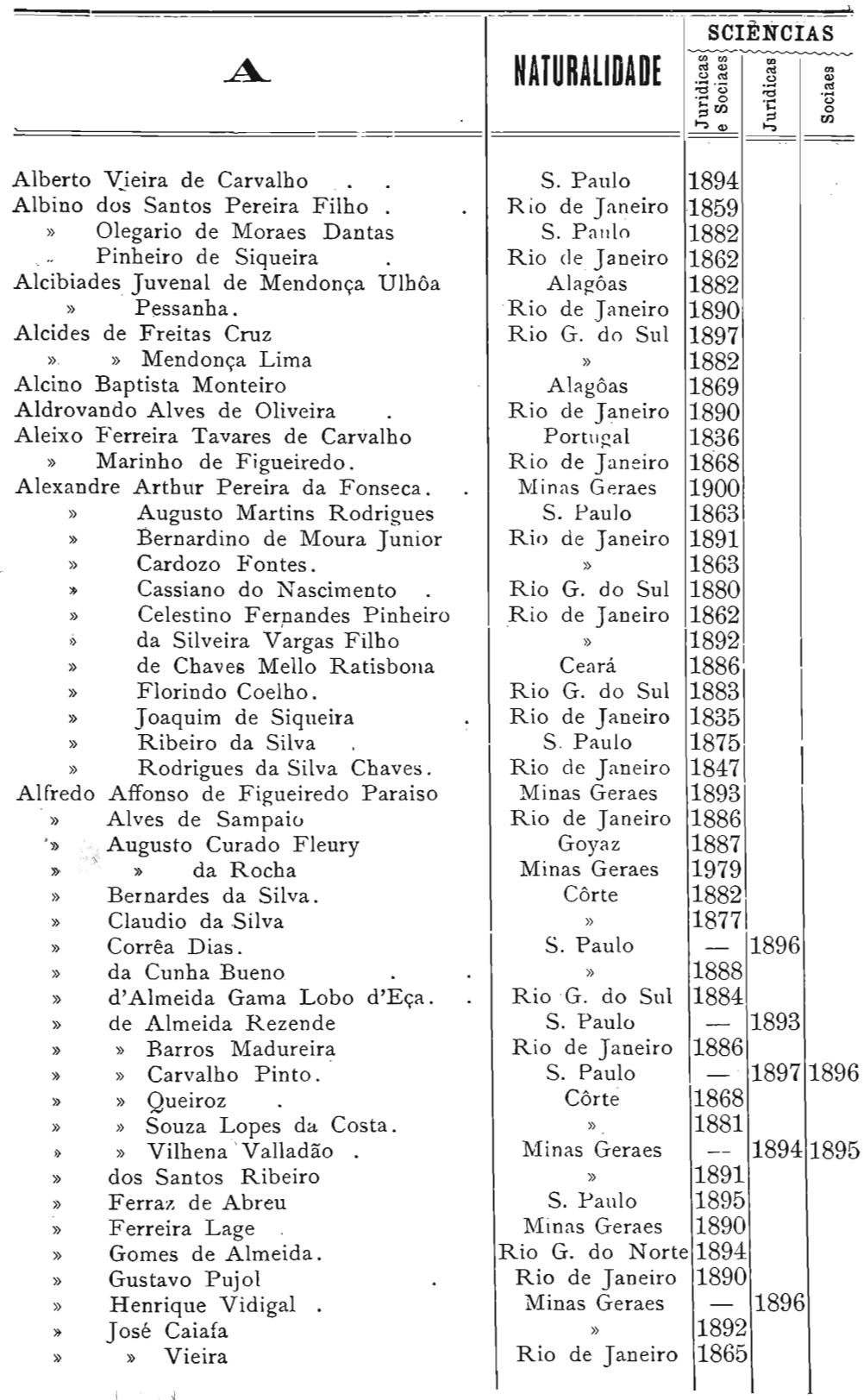




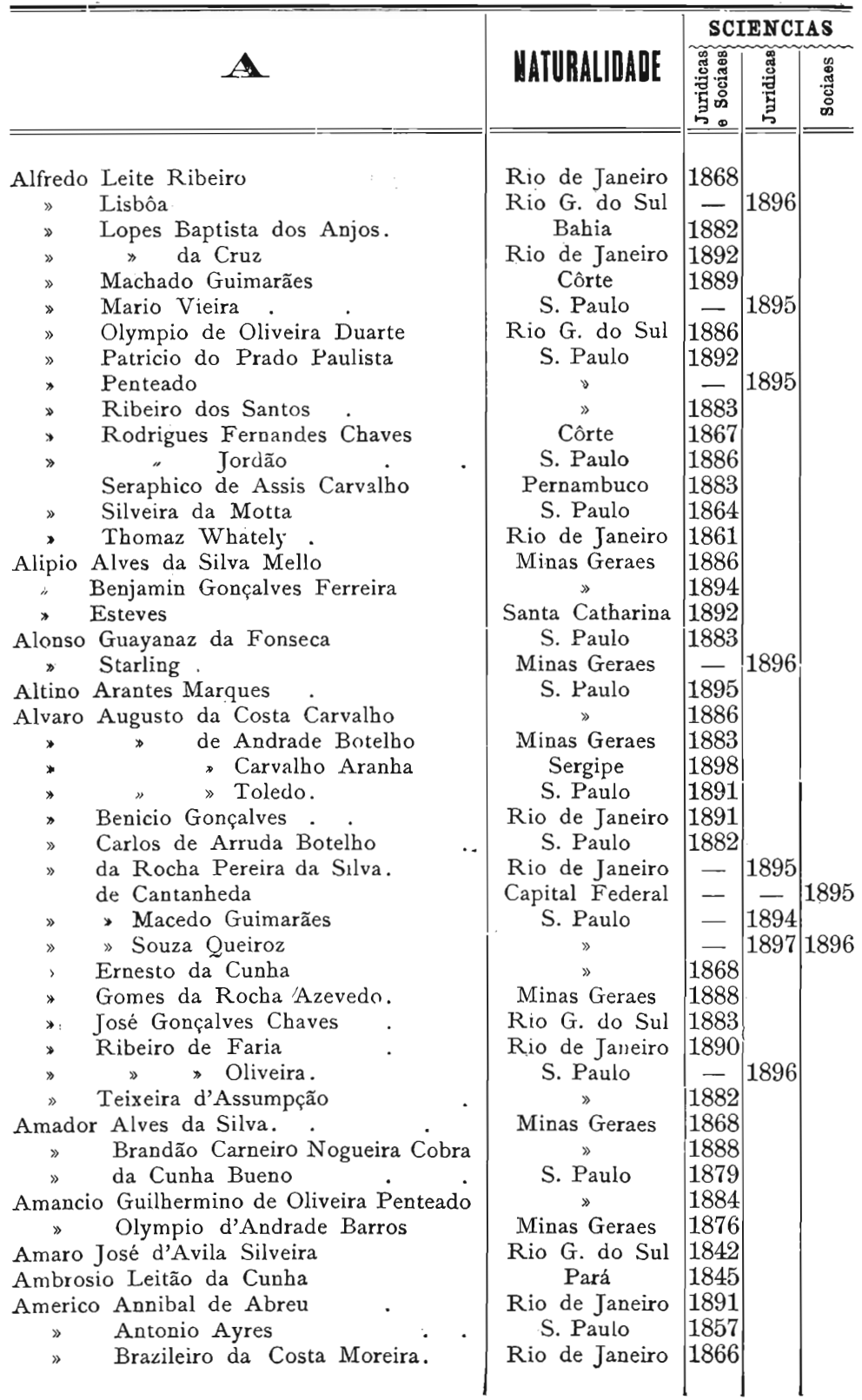




\begin{tabular}{|c|c|c|c|c|}
\hline & \multirow[b]{2}{*}{ NATURALIDADE } & \multicolumn{3}{|c|}{ SCIENCIAS } \\
\hline & & 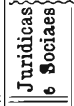 & 莺 & 胥 \\
\hline Americo Braziliense d'Almeida Mello. & S. Paulo & 1855 & & \\
\hline$\gg$ Brazilio de Campos & $\gg$ & 1860 & & \\
\hline Candido Tavares Bastos & Alagóas & 1862 & & \\
\hline Cantidiano Nogueira & Minas Geraes & 1873 & & \\
\hline de Campos Sobrinho & S. Paulo & 1891 & & \\
\hline » Moura Marcondes de Andrade & $\gg$ & 1857 & & \\
\hline » Oliveira Monteiro de Barros. & Minas Geraes & 1860 & & \\
\hline Ferreira de Abreu & S. Paulo & 1858 & & \\
\hline Lobo Leite Pereira & Minas Geraes & 1863 & & \\
\hline Ludolf . & Rio de Janeiro & 1889 & & \\
\hline Muniz Cordeiro Gitagy & Bahia & 1859 & & \\
\hline 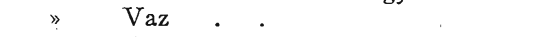 & S. Paulo & 1888 & & \\
\hline Vespucio Pinheiro e Prado & $»$ & 1860 & & \\
\hline » Xavier Pinheiro e Prado & $»$ & 1886 & & \\
\hline Anacleto José Ribeiro Coutinho & Rio de Janeiro & 1833 & & \\
\hline Anastacio Teixeira da Silva Bittencourt & S. Paulo & 1868 & & \\
\hline André Augusto de Padua Fleury & Matto Grosso & 1853 & & \\
\hline » Cordeiro de Araujo Lima & Rio de Janeiro & 1853 & & \\
\hline Corsino Pinto Chichorro da Gama. & Bahia & 1835 & & \\
\hline de Faro Fleury & Capital Federal & 1892 & & \\
\hline$» \quad$ Dias d'Aguiar & S. Paulo & 1857 & & \\
\hline » Martins d'Andrade & Minas Geraes & 1868 & & \\
\hline Junior & $»$ & 1893 & & \\
\hline Pereira Lima. & Bahia & 1833 & & \\
\hline Angelo Gomes Pinheiro Machado & Rio G. do Sul & 1882 & & \\
\hline » Guarinello & Italia & $\div$ & 1897 & \\
\hline » José da Fonseca & Rio de Janeiro & 1833 & & \\
\hline Mendes de Almeida & S. Paulo & 1896 & & \\
\hline Vieira Martins. & Minas Geraes & 1883 & & \\
\hline Antenor Augusto de Araujo & $»$ & 1886 & & \\
\hline Antero de Andrade Botelho & & 1893 & & \\
\hline$»$ Fernandes Cassalbo de Oliveira & Rio de Janeiro & 1872 & & \\
\hline Ferreira d'Avila. & Rio G. do Sul & 1867 & & \\
\hline José Lage Barbosa & Minas Geraes & 1856 & & \\
\hline Simões da Silva Cuim Atuá & Bahia & 1860 & & \\
\hline Victor da Silva Costa Pessôa. & S. Paulo & 1864 & & \\
\hline Antonino Carmelino de Mesquita Barros & » & 1886 & & \\
\hline$»$ do Amaral Vieira & $»$ & 1890 & & \\
\hline Antonio Achilles de Miranda Varejão. & Rio de Janeiro & 1856 & & \\
\hline » Affonso de Aguiar Witacker. & S. Paulo & 1859 & & \\
\hline$» \quad$ Lamonier Godofredo. & Minas Geraes & 1883 & & \\
\hline Ágostinho José da Silva & $»$ & 1865 & & \\
\hline Alberto de Almeida Corrêa & S. Paulo & 1889 & & \\
\hline Alexandrino Diniz. & Minas Gerees & 1891 & & \\
\hline Alonso de Faria & Rio de Janeiro & 1866 & & \\
\hline Alvares de Abreu e Silva Junior. & Minas Geraes & 1864 & & \\
\hline$» \quad$ Lobo & S. Paulo & 1884 & & \\
\hline
\end{tabular}




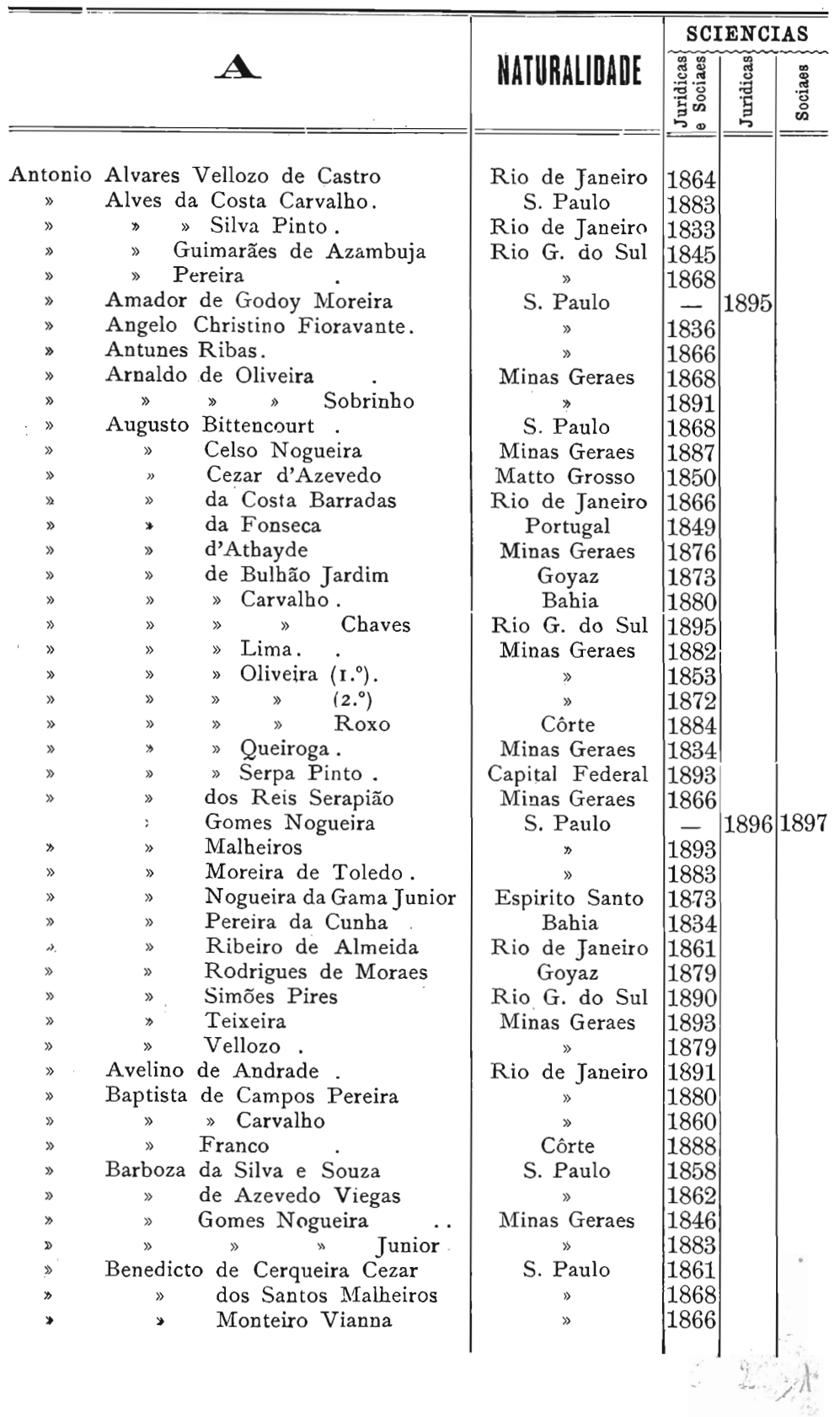




\begin{tabular}{|c|c|c|c|c|c|}
\hline & \multirow{2}{*}{$\Delta$} & \multirow[b]{2}{*}{ NATURALIDADE } & \multicolumn{3}{|c|}{ SCIENCIAS } \\
\hline & & & 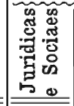 & 思 & 总 \\
\hline Antonio & Benedicto Valladares Ribeiro & Minas Geraes & 1895 & & \\
\hline. & Bento de Souza e Castro. & S. Paulo & 1868 & & \\
\hline$»$ & $»$ Dómingues de Castro. & $»$ & 1881 & & \\
\hline$"$ & Bittencourt Amarante Junior. & Minas Geraes & 1882 & & \\
\hline$»$ & Bley $\quad$. & Paraná & 1875 & & \\
\hline$»$ & Caetano de Oliveira Carvalho & S. Paulo & 1858 & & \\
\hline$\gg$ & Caio da Silva Prado. & $»$ & 1879 & & \\
\hline$»$ & Candido da Cunha Leitão & Rio de Janeiro & 1868 & & \\
\hline$»$ & $» \quad$ Rocha . & $\gg$ & 1845 & & \\
\hline$»$ & de Almeida e Silva. & S. Paulo & 1868 & & \\
\hline$»$ & de Azambuja. & Rio de Janeiro & 1868 & & \\
\hline$»$ & do Amaral. & S. Paulo & 1874 & & \\
\hline$»$ & $» \quad$ Ferreira de Abreu & $»$ & 1845 & & \\
\hline » & Teixeira & Minas Geraes & 1863 & & \\
\hline$»$ & Vieira & S. Paulo & 1886 & & \\
\hline$»$ & Xavier de Almeida Souza & $\gg$ & 1887 & & \\
\hline$»$ & Cardoso de Gusmão & Pernambuco & 1887 & & \\
\hline 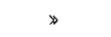 & Carlos Carneiro Viriato Catão & Minas Geraes & 1854 & & \\
\hline$»$ & » da Costa Carvalho Junior & Rio de Janeiro & 1891 & & \\
\hline$»$ & $» \quad$ Rocha Fragoso & Minas Geraes & - & 1895 & 1894 \\
\hline$»$ & » de Assumpção. & S. Paulo & - & 1894 & 1896 \\
\hline$"$ & » Castro Madeira & Pernambuco & 1880 & & \\
\hline$»$ & $» \quad »$ Moraes Salles & S. Paulo & 1866 & & \\
\hline$»$ & dos Reis Junior & Bahia & $\mid 1856$ & & \\
\hline$»$ & » Pereira da Cunha & Rio G. do Sul & - & 1895 & \\
\hline$\ggg$ & » Ribeiro de Andrada & S. Paulo & 1862 & & \\
\hline$»$ & $» \quad$ Filbo & Minas Geraes & 1891 & & \\
\hline$»$ & 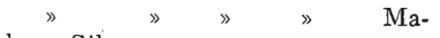 & & & & \\
\hline $\operatorname{cha}$ & do e Silva & S. Paulo & 1855 & & \\
\hline Antonio & Carlos Simões da Silva & Capital Federal & 1893 & & \\
\hline$»$ & Carneiro de Campos & S. Paulo & 1853 & & \\
\hline$»$ & Carvalho Serra. & Maranhão & 1866 & & \\
\hline$»$ & Casemiro da Motta Pacheco & Minas Geraes & 1860 & & \\
\hline$»$ & Celestino Soares & S. Paulo & 1884 & & \\
\hline$»$ & Cesario de Faria Alvim & Minas Geraes & 1865 & & \\
\hline$»$ & Cordeiro de Negreiros Lobato & $»$ & 1867 & & \\
\hline$»$ & Corrêa de Oliveira. & Rio G. do Sul & 1863 & & \\
\hline$»$ & $»$ do Couto & Matto Grosso & 1857 & & \\
\hline$»$ & Coutinho de Souza & Bahia & 1867 & & \\
\hline$\gg$ & Chrispiniano Barbosa Freire & Rio de Janeiro & 1884 & & \\
\hline$»$ & da Costa Pinto e Silva & $\gg$ & 1849 & & \\
\hline$»$ & Daniel Tanajura Guimarães & Bahia & 1884 & & \\
\hline$»$ & da Rocha Fernandes Leão & Minas Geraes & 1861 & & \\
\hline$\Rightarrow$ & »Rosa Moraes & S. Paulo & 1898 & & \\
\hline$»$ & » Sılva Corrêa & Rio de Janeiro & 1893 & & \\
\hline \# & $» \quad$ Jarckim & & 1882 & & \\
\hline$»$ & $» \quad » \quad$ Neves & Pernambuco & 1834 & & \\
\hline
\end{tabular}




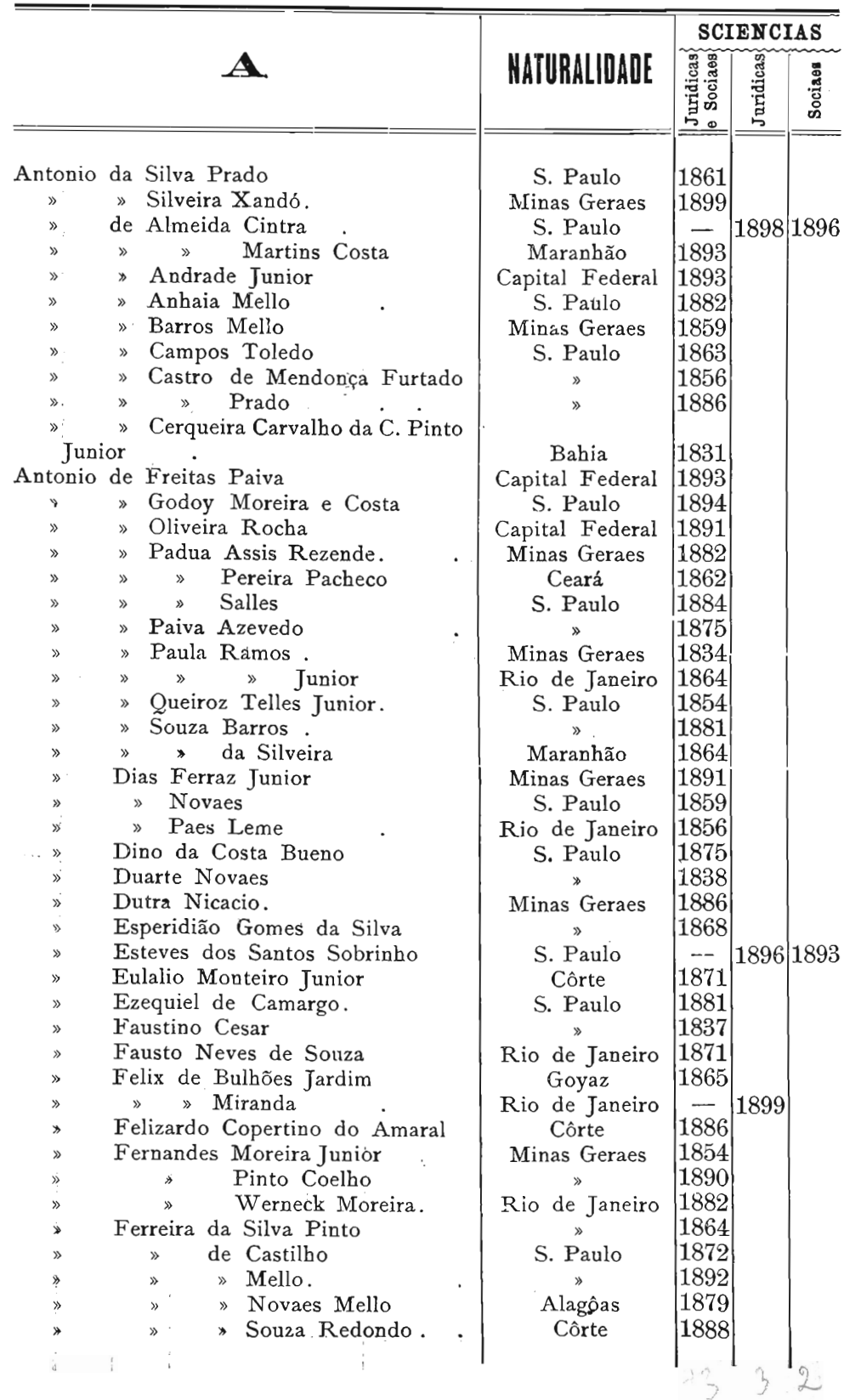




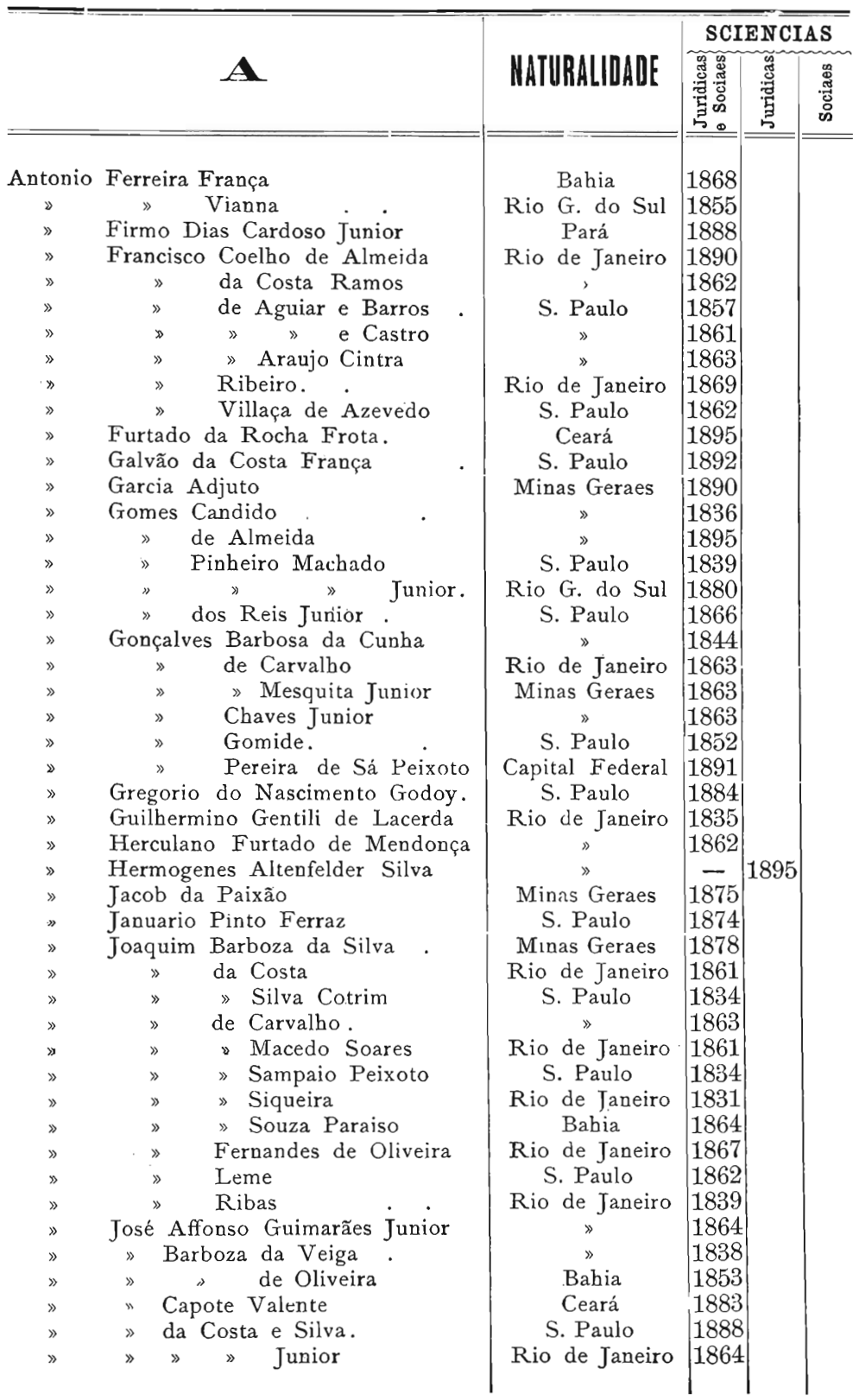




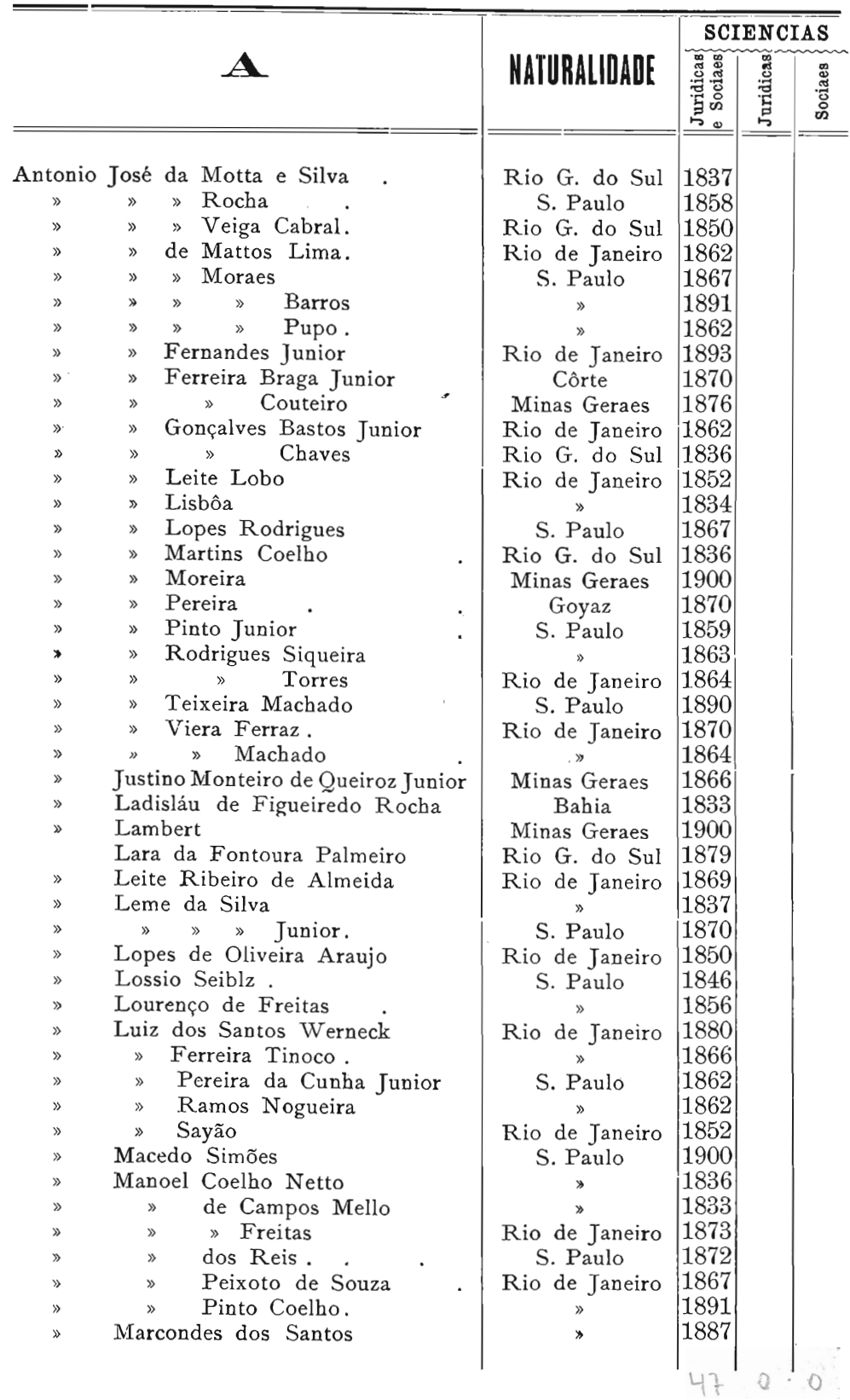




\begin{tabular}{|c|c|c|c|c|c|c|}
\hline & \multirow[b]{2}{*}{ YATIRALIDADE } & \multicolumn{3}{|c|}{ SCIENCIAS } \\
\hline & & & & $=$ & 管 & 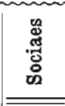 \\
\hline Antonio & Maria da Silva & & S. Paulo & 1881 & & \\
\hline$»$ & » Honorato Mercado. & & Rio G. do Sul & 1884 & & \\
\hline$»$ & Neves da Silveira (D. & & Rio de Janeiro & 1853 & & \\
\hline$\gg$ & Marinho Loureiro Chaves & & Rio G. do Sul & 1893 & & \\
\hline$»$ & Marques de Oliveira & & Minas Geraes & 1894 & & \\
\hline$»$ & Martins da Silva & & $\gg$ & - & 1895 & \\
\hline$»$ & $»$ Fontes Junior & & S. Paulo & 1899 & & \\
\hline$»$ & $»$ Torres . & & Rio de Janeiro & 1862 & & \\
\hline$»$ & Maximo do Couto & & Portugal & 1847 & & \\
\hline$»$ & $» \quad$ Nogueira Penido & & Minas Geraes & 1867 & & \\
\hline$\gg$ & Ribeiro da Luz. & & $»$ & 1848 & & \\
\hline$»$ & Mendes de Oliveira Castro & So- & & & & \\
\hline brin & ho . . & & Côrte & 1886 & & \\
\hline Antonio & Militão de Souza Aymberé. & & S. Paulo & 1889 & & \\
\hline$»$ & Moniz Barreto. . . & & Bahia & 1837 & & \\
\hline$»$ & $»$ de Souza & & S. Paulo & 1878 & & \\
\hline$»$ & Monteiro Freire & & Rio de Janeiro & 1881 & & \\
\hline$»$ & Moreira de Barros & & S. Paulo & 1861 & & \\
\hline$»$ & $» \gg$ Filho. & & & 1888 & & \\
\hline$»$ & $» \quad$ Tavares & & Rio de Janeiro & 1856 & & \\
\hline$»$ & Navarro de Abreu & & Matto Grosso & 1835 & & \\
\hline$»$ & Nunes de Aguiar Junior. & & Rio de Janeiro & 1850 & & \\
\hline$»$ & " „ Seabra Perestrello. & & & 1856 & & \\
\hline$»$ & » Gomes Pereira & & Rio G. do Sul & 1888 & & \\
\hline$»$ & Olavo de Araujo. & & Bahia & 1869 & & \\
\hline$»$ & Olympio Rodrigues Vieira & & Ceará & - & 1895 & \\
\hline '» & Pacheco Ferreira Vianna. & & Côrte & 1883 & & \\
\hline$»$ & Paes de Barros Sobrinho. & . & S. Paulo & 1886 & & \\
\hline$»$ & Passos de Miranda Filho & & Pará & 1891 & & \\
\hline$»$ & Paulino da Silva & & S. Paulo & 1891 & & \\
\hline$"$ & » Soares de Souza. & & Côrte & 1865 & & \\
\hline$»$ & Pedro da Costa Pinto & & Minas Geraes & 1860 & & \\
\hline$»$ & d'Alencastro Junior & & Côrte & 1870 & & \\
\hline$\gg$. & $\bowtie$ de Souza e Silva & . & Rio de Janeiro & 1882 & & \\
\hline$\gg$ & » Monteiro de Souza & & $»$ & 1864 & & \\
\hline » & Pereira da Rocha Soares. & . & S. Paulo & 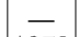 & - & 1893 \\
\hline$»$ & $» \quad »$ Silva Barros & & $»$ & 1872 & & \\
\hline 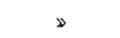 & $» \quad$ de Abreu Junior & • & Goyaz & - & 1898 & \\
\hline > & $\gg \quad » Q$ Queiroz & & S. Paulo & 1883 & & \\
\hline$\gg$ & $»$ dos Santos & & $»$ & 1857 & & \\
\hline$»$ & Pimentel Junior & & $»$ & 1898 & & \\
\hline$》$ & Pinbeiro de Albuquerque. & & $»$ & 1887 & & \\
\hline$\gg$ & Pinto de Almeida Ferraz & & $》$ & 1891 & & \\
\hline$»$ & $\gg \gg$ Oliveira & & Minas Geraes & 1891 & & \\
\hline$»$ & $»$ do Rego Freitas & & S. Paulo & 1861 & & \\
\hline$»$ & Proost Rodovalho Junior & & $»$ & 1890 & & \\
\hline » & Quirino de Souza e Castro & & $»$ & 1860 & & \\
\hline
\end{tabular}




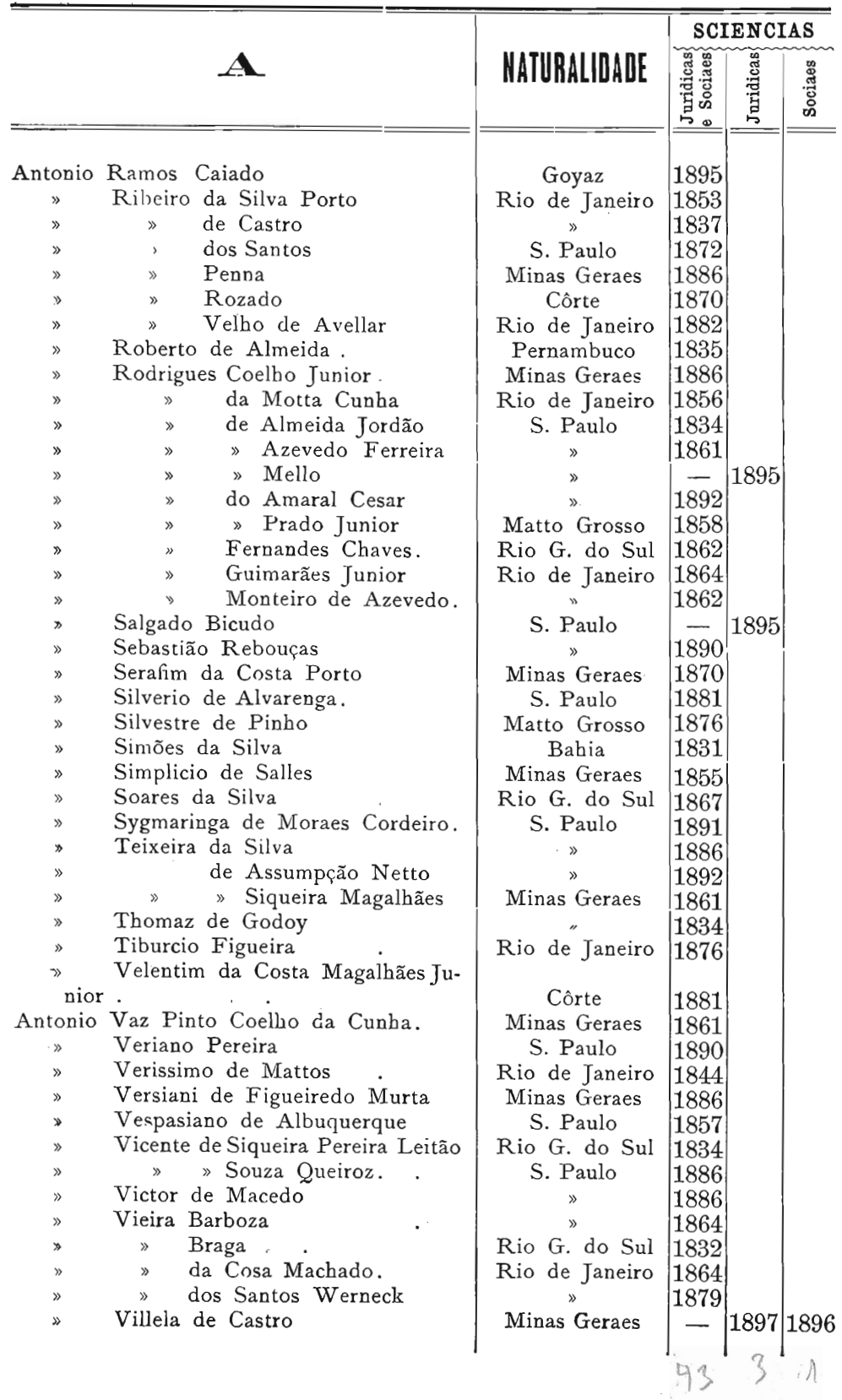




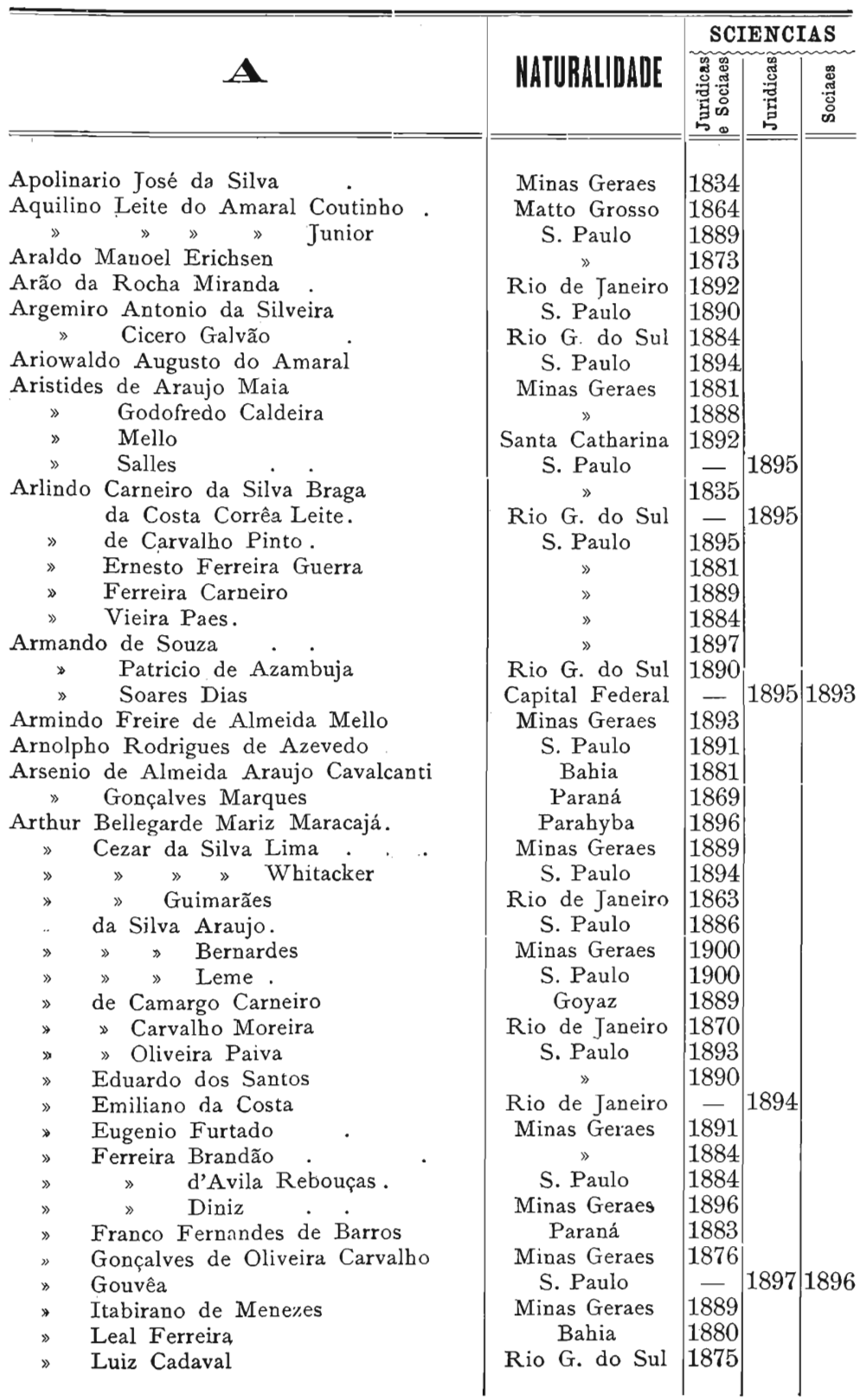




\begin{tabular}{|c|c|c|c|c|}
\hline \multirow{2}{*}{$\Delta$} & \multirow[b]{2}{*}{ NATURALIDADE } & \multicolumn{3}{|c|}{ SCIENCIAS } \\
\hline & & 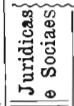 & 苟 & 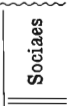 \\
\hline Arthur Mesquita Curtines Laxe & Rio de Janeiro & 1888 & & \\
\hline M Moreira de Almeida & S. Paulo & {$\left[\begin{array}{c}1000 \\
-\end{array}\right.$} & 1894 & \\
\hline$» \quad »$ Castro Lima & $»$ & 1889 & & \\
\hline » Nicolau de Vergueiro & $»$ & 1884 & & \\
\hline$"$ Octaviano Braga & Rio G. do Sul & 1879 & & \\
\hline Pinto Lima & Rio de Janeiro & 1898 & & \\
\hline Prado de Queiroz Telles & S. Paulo & 1884 & & \\
\hline Ribeiro de Oliveira & Minas Geraes & 1888 & & \\
\hline Rudge da Silva Ramos & S. Paulo & & 1898 & 1896 \\
\hline Severiano Ferreira Guimarães & Minas Geraes & 1888 & & \\
\hline Soarıs de Moura & $»$ & 1892 & & \\
\hline Teixeira Leite & $\gg$ & 1870 & & \\
\hline - Travassos Prestes & Rio G. do Sul & 1893 & & \\
\hline V Vautier. & S. Paulo & 1888 & & \\
\hline Vianna Barbosa. & Rio de Janeiro & 1900 & & \\
\hline ¿ Xavier Pinbeiro e Prado & Rio G. do Sul & 1892 & & \\
\hline Ascanio Beriguy. & S. Paulo & $\ldots$ & & 1896 \\
\hline$\gg \quad »$ de Cerqueira. & $»$ & - & 1897 & \\
\hline Ascendino Angelo dos Reis (Dr.) & Sergipe & 1889 & & \\
\hline Astolpho Dutra Nicacio & Minas Geraes & 1888 & & \\
\hline , Pio da Silva Pinto & $»$ & 1861 & & \\
\hline Vieira de Rezende e Silva & $»$ & 1891 & & \\
\hline Ataliba Leonel Rolim & S. Paulo & - & 1896 & 1895 \\
\hline Ataulfo Napoles de Paiva & Rio de Janeiro & 1887 & & \\
\hline Atto Chaves Barcellos & Rio r. do Sul & 1891 & & \\
\hline Augusto Albino de Almeida & Minas Geraes & 1891 & & \\
\hline * Alvares de Azevedo & Côrte & 1867 & & \\
\hline Balthasar da Silveira & Bahia & 1834 & & \\
\hline Cesar de Barros Cruz & S. Paulo & 1884 & & \\
\hline$\gg \quad$ Mattos. & $\gg$ & 1883 & & \\
\hline$\gg \quad »$ Padua Fleury & Goyaz & 1860 & & \\
\hline Cincinato de Almeida Líma & S. Faulo & 1859 & & \\
\hline Cochrane de Alencar & Côrte & 1888 & & \\
\hline da Costa Guimarães & S. Paulo & 1894 & & \\
\hline de Azevedo Vianna & Minas Geraes & 1893 & & \\
\hline »Meirelles Reis. & S. Paulo & 1886 & & \\
\hline$»$ Oliveira Maia. & Rio de Janeiro & 1891 & & \\
\hline »Siqneira Cardoso & S. Paulo & 1881 & & \\
\hline »Souza Queiroz. & $»$ & 1872 & & \\
\hline do Couto Delgado & Côrte & 1869 & & \\
\hline Fausto Guimarães Alvim. & Minas Geraes & 1857 & & \\
\hline $\begin{array}{cl}\text { Ferreira de Castilbo } & \text { de } \\
\text { França }\end{array}$ & $\begin{array}{l}\text { S. Paulo } \\
\text { Bahia }\end{array}$ & $\overline{1857}$ & 18 & 1895 \\
\hline Freire da Silva & Maranhão & 1862 & & \\
\hline \» $\gg$ Junior & S. Paulo & 1884 & & \\
\hline ఎ de Andrade & Minas Geraes & 1892 & & \\
\hline Henrique Turk & S. Paulo & 1894 & & \\
\hline
\end{tabular}




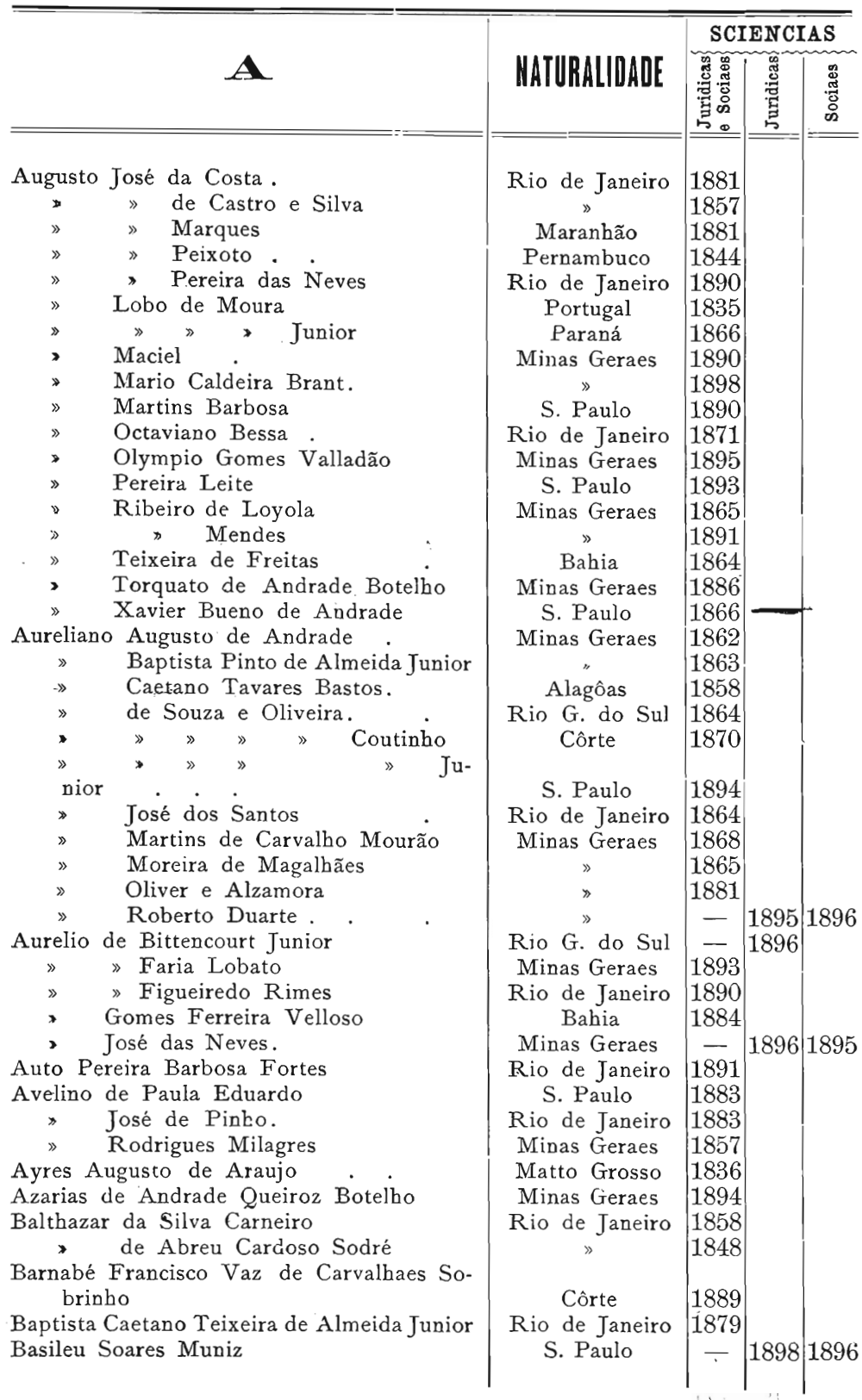




\begin{tabular}{|c|c|c|c|c|c|}
\hline \multirow{2}{*}{\multicolumn{2}{|c|}{$1 B$}} & \multirow[b]{2}{*}{ NATURALIIAALE } & \multicolumn{3}{|c|}{ SCIENCIAS } \\
\hline & & & 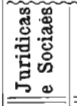 & 䔅 & 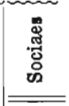 \\
\hline \multicolumn{2}{|c|}{ Belisario Francisco Caldas. } & Rio de Janeiro & 1869 & & \\
\hline \multirow{2}{*}{\multicolumn{2}{|c|}{ " Pereira de Carvalho }} & S. Paulo & 1900 & & \\
\hline & & $»$ & 1898 & & \\
\hline \multicolumn{2}{|c|}{$\gg$ Castilho de Andrade. } & 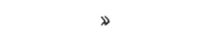 & 1889 & & \\
\hline \multicolumn{2}{|r|}{ » Cordeiro de Campos Valladares } & Minas Geraes & 1872 & & \\
\hline \multicolumn{2}{|r|}{ da Lapa Trancoso } & S. Paulo & & & 18 \\
\hline \multicolumn{2}{|c|}{ - Felix de Souza } & Goyaz. & 1859 & & \\
\hline \multicolumn{2}{|r|}{$\begin{array}{l}\text { Ferraz de Camargo . } \\
\text { Froscolo Tovino de Almeida }\end{array}$} & S. Paulo & 1894 & & \\
\hline \multirow{2}{*}{\multicolumn{2}{|c|}{$\begin{array}{l}\text { Froscolo Jovino de Almeida } \\
\text { Aymberé }\end{array}$}} & & & & \\
\hline & & Bahia & 1860 & & \\
\hline \multicolumn{2}{|c|}{ Benedicto José de Araujo Toledo. } & S. Paulo & $\begin{array}{l}1836 \\
1890\end{array} \mid$ & & \\
\hline \multicolumn{2}{|r|}{ » Philadelpho de Castro } & $»$ & 1880 & & \\
\hline \multicolumn{2}{|c|}{$» \quad$ Rolim Junior } & $»$ & - & 1897 & 18 \\
\hline \multicolumn{2}{|c|}{$\begin{array}{cl}\text { Benjamim Antunes Je Oliveira. } & \\
\text { " da Luz Novaes }\end{array}$} & Ceará & 1889 & & \\
\hline \multirow{2}{*}{\multicolumn{2}{|c|}{$» \quad$ Firmo de Paula Araueira }} & $\begin{array}{l}\text { S. Paulo } \\
\text { Sinas }\end{array}$ & $\begin{array}{l}1893 \\
1884\end{array}$ & & \\
\hline & & $\begin{array}{c}\text { Minas Geraes } \\
\text { „ }\end{array}$ & $\begin{array}{l}1884 \\
1886\end{array} \mid$ & & \\
\hline \multicolumn{2}{|c|}{ \ Rodrigues Pereira } & $»$ & 1858 & & \\
\hline \multicolumn{2}{|c|}{ Bento Aguiar de Barros } & S. Paulo & 1863 & & \\
\hline \multicolumn{2}{|c|}{$» \quad$ Antunes Barroso. } & Rio de Janeiro & 1872 & & \\
\hline \multicolumn{2}{|c|}{ » Barata Ribeiro } & S. Paulo & 1891 & & \\
\hline $\mathrm{B}$ & Benedicto Coelho de Almeida & Rio de Janeiro & 1889 & & \\
\hline $\mathrm{C}$ & Carneiro de Almeida Pereira & $\gg$ & 1880 & & \\
\hline $\mathrm{F}$ & Francisco de Paula e Souza & S. Paulo & 1857 & & \\
\hline $\mathrm{G}$ & Galvão da Costa e Silva & $»$ & 1886 & & \\
\hline$\gg \quad \mathrm{L}$ & Luiz de Oliveira Lisbôa. & Rio de Janeiro & 1859 & & \\
\hline$\gg$ & » $\quad$ Toledo Lisbôa & Côrte & 1888 & & \\
\hline M & Manoel de Almeida Baptista & Rio de Janeiro & 1859 & & \\
\hline $\mathrm{P}$ & Pereira Bueno . & S. Paulo & 1893 & & \\
\hline $\mathbf{P}$ & Pinto do Rego Freitas & $»$ & 1886 & & \\
\hline$»$ & » Ribeiro Pereira de Sampaio. & Rio de Janeiro & 1857 & & \\
\hline $\mathrm{R}$ & Ribeiro da Luz & Minas Geraes & 1891 & & \\
\hline$»$ & » dos Santos Camargo & S. Paulo & 1884 & & \\
\hline$» \quad \mathrm{R}$ & Rodrigues Freire. & Rio de Janeiro & 1868 & & \\
\hline Bernardi & lino Augusto de Lima & Minas Geraes & 1882 & & \\
\hline$»$ & de Souza Monteiro. & Espirito Santo & 1893 & & \\
\hline$»$ & Ferreira da Silva & S. Paulo & 1879 & & \\
\hline$»$ & Guerreiro Rodrigues Torres & Rio de Janeiro & 1891 & & \\
\hline$\gg$ & José de Aquino & Minas Greraes & 1838 & & \\
\hline$\gg$ & $\gg$ \Campos & Bahia & 1834 & & \\
\hline$»$ & $\star \quad » \quad$ Junior & Minas Geraes & 1863 & & \\
\hline$»$ & "Queiroga & $»$ & 1833 & & \\
\hline$»$ & $» \quad$ Rodrigues Ferreira & Rio G. do Sul & 1838 & & \\
\hline$»$ & Pamplona de Menezes. & Rio de Janeiro & 1869 & & \\
\hline$»$ & Peixoto de Campos & S. Paulo & 1893 & & \\
\hline Bernardo & Ho Augusto Nascentes de Azambuja & Rio de Janeiro & 1833 & & \\
\hline
\end{tabular}




\begin{tabular}{l}
\hline Bernardo Augusto Rodrigues da Silva \\
$»$
\end{tabular}

Boaventura Antonio da Costa

» Seraphico de Brito Guerra

Bogumil Bartholomey

Braulio Augusto de Bragariça

» Romulo Colonia

» Thimotbeo Urioste.

Braz Barbosa da Silva

» Odorico de Freitas

Brazilio Alves Corrêa do Amaral

- A Augusto Machado de Oliveira

" Rodrigues dos Santos

"Ytiberé da Cunba

Briano O'Connor de ('amargo Dauntre

Caetano Alves Rodrigues Horta

» Augusto da Gama Cerqueira

$\gg$ dos Santos . .

» Furquim de Almeida

» José de Andrade Pinto

» »Souza

» Luiz Machado de Magalhães

» Marques dos Santos

\Pinto de Miranda Montenegro

» Xavier da Silva Pereira

Caio de Campos Valladares.

Calimerio Nestor dos Santos.

Camillo Augusto Maria de Brito

» Gavião Peixoto.

» Soares de Moura Junior

Candido Alves Duarte Silva . » Pereira

Augusto Rodrigues.

Baptista de Lacerda

Eueno da Costa Junior

Carneiro Ribas

de Souza Campos

"Toledo Malta

Drummond Furtado de Mendonça.

- Fernando da Costa Guimarães Junior

\section{NATURALIDADE}

S. Paulo

"

Rio G. do Sul

Rio de Janeiro

Minas Geraes

Côrte

Minas Geraes

Rio de Janeiro

Rio G. do Sul

Rio G. do Norte

Rio G. do Sul

Rio de Janeiro

Babia

S. Paulo

»

$\gg$

$\gg$

$\gg$

Paraná

S. Paulo

Minas Geraes

$$
»
$$

Côrte

Minas Geraes

Rio de Janeiro Maranhão

Minas Geraes

Rio de Janeiro Côrte

Matto Grosso

Rio de Janeiro

Minas Geraes

S. Paulo

Minas Geraes

Rio de Janeiro

Rio G. do Sul

S. Paulo

Rio de Janeiro

Minas Geraes

S. Paulo

»

Rio de Janeiro Côrte

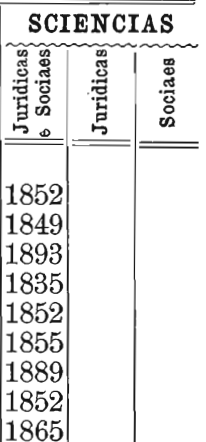

1879

1884

1890

1857

1858

1863

1880

1872

1877

1870

1879

1835

1867

1881

1838

1855

1853

1875

1850

1873

1859

1889

1865

1861

1889

1866

1834

1884

1874

1844

1884

1895

1884

1873

1873 


\begin{tabular}{|c|c|c|c|c|c|}
\hline \multirow{2}{*}{\multicolumn{2}{|c|}{$\mathrm{C}$}} & \multirow[b]{2}{*}{ NATIRALIDADE } & \multicolumn{3}{|c|}{ SCIENCIAS } \\
\hline & & & 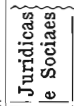 & 菢 & 恋 \\
\hline Candido & o Ferreira da Silva Camargo & S. Paulo & 1861 & & \\
\hline$\gg$ & Gomes de Vasconcellos Guanabara & Rio de Janeiro & 1855 & & \\
\hline » & José de Andrade. & S. Paulo & 1854 & & \\
\hline$》$ & » Mariano Junior & Minas Geraes & 1861 & & \\
\hline$»$ & » Rodrigues Torres & Rio de Janeiro & 1863 & & \\
\hline$》$ & Leopoldo da Motta Cruz. & $》$ & 1862 & & \\
\hline 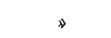 & Lopes de Oliveira. & S. Paulo & 1857 & & \\
\hline$\gg$ & Luiz Maria de Oliveira & Minas Geraes & 1865 & & \\
\hline$»$ & Monteiro da Cunha Bueno & S. Paulo & 1884 & & \\
\hline$»$ & Nanzianzeno Nogueira da Motta. & $»$ & 1891 & & \\
\hline ॥ & Pereira Barreto & Rio de Janeiro & 1860 & & \\
\hline$»$ & $»$ Gustavo & Minas Geraes & 1867 & & \\
\hline$»$ & Rebello de Araujo Palhares & S. Paulo & 1835 & & \\
\hline$»$ & Tavares Bastos & Rio G. do Sul & 1886 & & \\
\hline$»$ & Teixeira Fortes. & Minas Geraes & 1867 & & \\
\hline$»$ & Xavier de Almeida e Souza. & S. Paulo & 1854 & & \\
\hline Cantidio & o Tolentino de Figueiredo Bretas & Minas Geraes & 1896 & & \\
\hline Canuto & José Saraiva. & S. Paulo & 1875 & & \\
\hline Carlindo & lo dos Santos Pinto & Minas Geraes & 1890 & & \\
\hline Carlos & Affonso de Assis Figueiredo & $\gg$ & 1867 & & \\
\hline$\gg I$ & Albertn de Bulbões Ribeiro & S. Paulo & 1868 & & \\
\hline$»$ & » Teixeira Leite & Minas Geraes & 1862 & & \\
\hline 》 & Vianna & S. Paulo & 1899 & & \\
\hline$»$ & Antonio Cordeiro & Rio de Janeiro & 1834 & & \\
\hline$»$ & $» \quad$ de Bulhões Ribeiro & . & 1833 & & \\
\hline$》$ & $» \quad$ "Carvalbo & $"$ & 1836 & & \\
\hline$»$ & $» \quad$ \#rança Carvalbo & $»$ & 1867 & & \\
\hline$»$ & $\triangleq \quad$ Rodrigues. & S. Paulo & 1862 & & \\
\hline$»$ & Arthur Busch Varella. & Rio de Janeiro & 1848 & & \\
\hline$»$ & Augusto Cardozo de Menezes & S. Paulo & 1866 & & \\
\hline$》$ & $»$ Coelho & Capital Federal & - & 1895 & 1893 \\
\hline$»$ & $» \quad$ de Carvalho & Côrte & 1873 & & \\
\hline$»$ & "Freitas Villalva & S. Paulo & 1880 & & \\
\hline$»$ & » Oliveira Figueiredo & Rio de Janeiro & 1858 & & \\
\hline 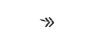 & $» \quad »$ Souza Lima. & S. Paulo & 1866 & & \\
\hline$»$ & $»$ do Amaral Sobrinho & ( & 1866 & & \\
\hline$»$ & Ferreira Brandão & Minas Geraes & 1892 & & \\
\hline$»$ & Garcia Ferreira & S. Paulo & 1882 & & \\
\hline$"$ & Germano Knüppeln & Rio G. do Sul & 1895 & & \\
\hline$»$ & » Pereira Guimarães. & S. Paulo & 1883 & & \\
\hline$»$ & Baptista de Castro & Minas Geraes & 1861 & & \\
\hline » & Borges Monteiro. & Côrte & 1886 & & \\
\hline$»$ & Caetano de Abreu & S. Paulo & 1863 & & \\
\hline » & Canuto Malheiros & $\gg$ & 1851 & & \\
\hline$»$ & Carneiro de Barros Azevedo & Côrte & 1876 & & \\
\hline$»$ & Coelho de Oliveira & Capital Federal & 1890 & & \\
\hline$»$ & de Arruda Botelho & S. Paulo & 1891 & & \\
\hline
\end{tabular}




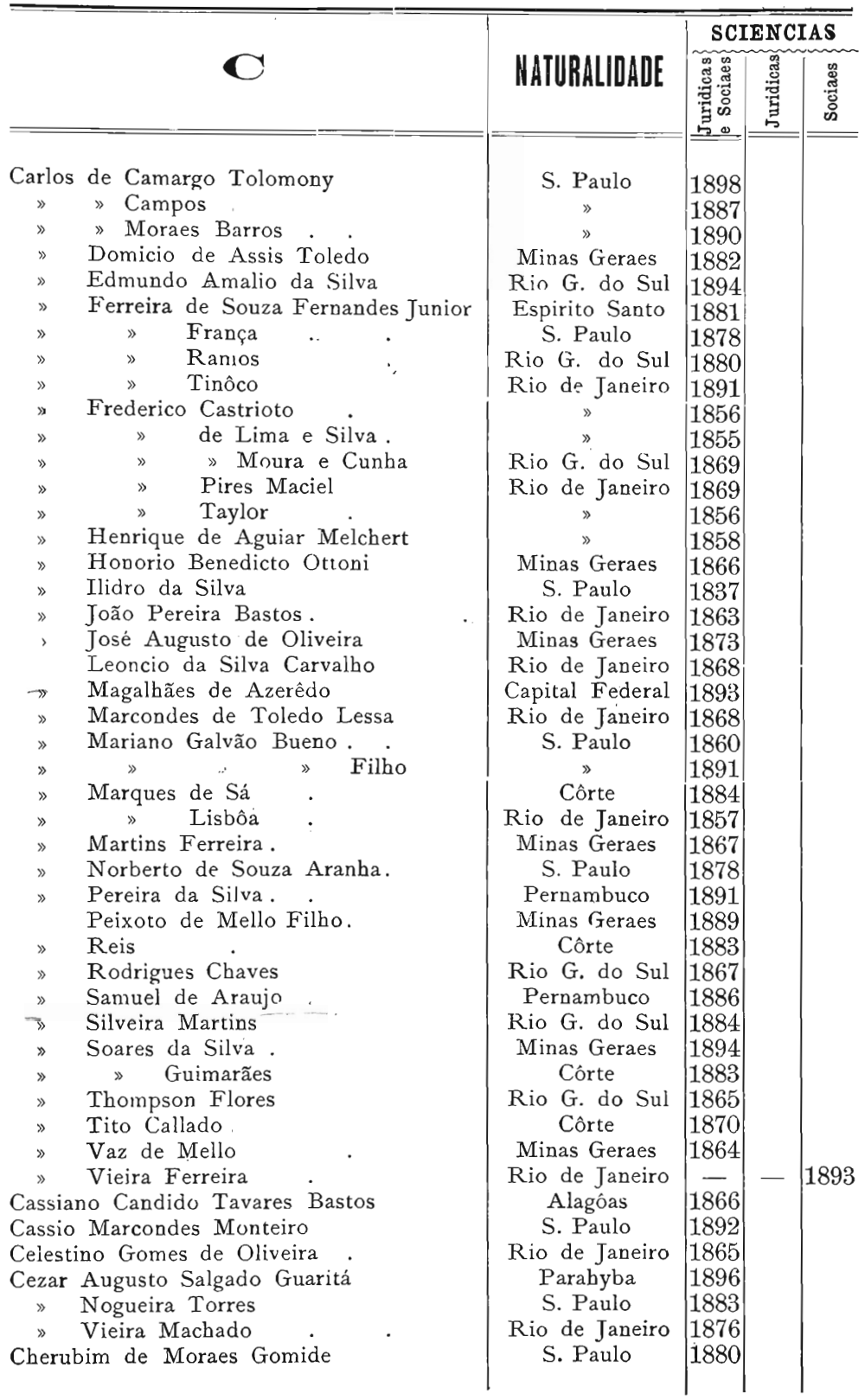




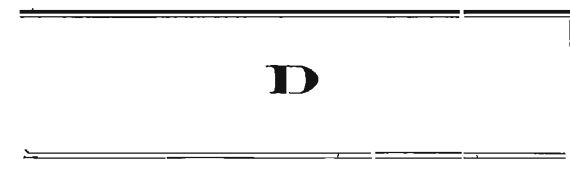

Cherubim Ferraz de Andrade

Chrispim Jacques de Bias Fortes

Christiano Alberto Vianna Ritt

$$
\text { » de Macedo Costa }
$$

» Mauricio Stockler de Lima.

» Pereira Brazil

» Vieira de Andrade

Christovão Corrêa e Castro.

$$
\text { Rodrigues de Andrade. }
$$

Cicero Eurico Leonel

» Ribeiro de Castro

Cincinato Cezar da Silva Braga

Claudino Pereira da Fonseca

$$
\text { » Teixeira Guimarães }
$$

Claudio Herculano Duarte " Jeronymo Stockler de Lima

Clemente Falcão de Souza Filbo. ग José Ferreira Braga Junior

Clementino de Suuza Castro

$$
\text { » José do Carmo Junior }
$$

Cleophano Pitaguary de Araujo

$$
\text { 》, " Mello Terra }
$$

Conrado Caetano Ericbsen

Constancio Rodrigues da Silveira

Constantino de Almeida Faria

». Ernesto de Figueiredo Faro

» Gonçalves Fraga

\osé Gonçalves.

» Luiz Paleta

Crescencio José de Oliveira Costa

Custodio Alves dos Santos.

» Cardoso Fontes Filho

» Celso de Saboia e Silva

» de Araujo Padilha

" José Coelho de Almeida.

» da Costa Cruz

" Leite de Souza

» Mercellino de Magalhães.

\begin{tabular}{|c|c|c|c|}
\hline \multirow[b]{2}{*}{ NATURALIDADE } & \multicolumn{3}{|c|}{ SCIENCIAS } \\
\hline & 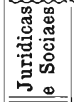 & 學 & 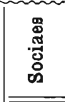 \\
\hline S. Paulo & 1886 & & \\
\hline Minas Geraes & 1870 & & \\
\hline Côrte & 1881 & & \\
\hline S. Paulo & - & 1895 & 1896 \\
\hline Minas Geraes & 1858 & & \\
\hline$»$ & 1858 & & \\
\hline$»$ & 1886 & & \\
\hline & 1886 & & \\
\hline Rio de Janeiro & 1860 & & \\
\hline 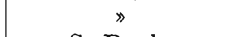 & 1857 & & \\
\hline S. Paulo & 1900 & & \\
\hline Minas Geraes & 1893 & & \\
\hline S. Paulo & |1886 | - & & \\
\hline Minas Geraes & 1858 & & \\
\hline Portugal & 1839 & & \\
\hline Minas Geraes & 1863 & & \\
\hline S. Paulo & 1861 & & \\
\hline$»$ & 1855 & & \\
\hline Rio de Janeiro & 1861 & & \\
\hline S. Paulo & 1876 & & \\
\hline Minas Geraes & 1868 & & \\
\hline Bahia & 1874 & & \\
\hline Minas Geraes & 1890 & & \\
\hline$»$ & 1888 & & \\
\hline S. Paulo & 1868 & & \\
\hline$》$ & 1891 & & \\
\hline$»$ & 1839 & & \\
\hline$»$ & 1884 & & \\
\hline Bahia. & 1887 & & \\
\hline Rio de Janeiro & 1863 & & \\
\hline Minas Geraes & 1884 & & \\
\hline S. Paulo & 1873 & & \\
\hline Piauby & 1862 & & \\
\hline Rio de Janeiro & 1860 & & \\
\hline Ceará & $-1 \overline{00}$ & - & 1894 \\
\hline Minas Geraes & 1860 & & \\
\hline Rio de Janeiro & 1886 & & \\
\hline Minas Geraes & 1865 & & \\
\hline Rio de Janeiro & 1862 & & \\
\hline$»$ & 1862 & & \\
\hline Minas Geraes & 1850 & & \\
\hline Pernambuco & 1849 & & \\
\hline Rio de Janeiro & 1835 & & \\
\hline S. Paulo & 1863 & & \\
\hline Minas Geraes & 1832 & & \\
\hline Sergipe & 1882 & & \\
\hline S. Paulo & 1883 & & \\
\hline
\end{tabular}

» Rodrigues de Moura

Cypriano Fenelon Guedes Alcanforado

$$
\begin{gathered}
» \text { José Lisbôa } \\
» \quad » \text { Soares }
\end{gathered}
$$

Cyrino Antonio de Lemos

Cyro Franklim de Azevedo.

Damaso Candido Corrêa Coelbo 


\begin{tabular}{|c|c|c|c|c|}
\hline \multirow{2}{*}{ IE } & \multirow[b]{2}{*}{ NATURALIDADE } & \multicolumn{3}{|c|}{ SCIENCIAS } \\
\hline & & 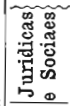 & 莺 & 丞 \\
\hline Domingos José Vaz Dias Junior & Rio G. do Sul & - & 1897 & 1898 \\
\hline Licinio Ferraz & S. Paulo & $\overline{1894}$ & & \\
\hline Marcondes de Andrade & Rio de Janeiro & 1866 & & \\
\hline Pinto França Mascarenbas Junior & 》 & 1859 & & \\
\hline Ramos Mello Junior. & ». & 1865 & & \\
\hline Theodoro de Mendonça. & Minas Geraes & 1870 & & \\
\hline 》 Velho Pereira da Veiga. & Rio de Janeiro & 1836 & & \\
\hline Donato Joaquim da Fonseca & Minas Geraes & $\begin{array}{l}1000 \\
1887\end{array}$ & & \\
\hline Edgard de Almeida Prado & S. Paulo & & 1896 & 1895 \\
\hline " Novaes Carvalho & Pernambuco & $\overline{1895}$ & & \\
\hline Edgardo Carlos da Cunba Pereira. & Minas Geraes & 1884 & & \\
\hline " Guilherme Pahl & Capital Federal & 1898 & & \\
\hline Edmundo Muniz Barreto & Côrte & 1884 & & \\
\hline » Palmeiro Pereira da Cunha. & Rio G. do Sul & 1876 & & \\
\hline Pereira Lins & Minas Geraes & 1889 & & \\
\hline $\begin{array}{cc}{ }^{»} & \text { Veiga } \\
\text { Edmur de } & \text { Souza }\end{array}$ & Sergipe & 1889 & & \\
\hline $\begin{array}{l}\text { Edmur de Souza Queiroz } \\
\text { Eduardo Aleixo Callado }\end{array}$ & S. Paulo & 1900 & & \\
\hline $\begin{array}{ll} & \\
& \text { Alves Guimarães }\end{array}$ & $\begin{array}{l}\text { Montevidéo } \\
\text { Côrte }\end{array}$ & $\begin{array}{l}1854 \\
1889\end{array}$ & & \\
\hline Antonio de Barros & Minas Geraes & $\begin{array}{l}1809 \\
1862\end{array}$ & & \\
\hline Augusto Nogueira de Camargo & S. Paulo & 1880 & & \\
\hline Barbosa Nogueira. & Minas Geraes & 1886 & & \\
\hline Camargo Neves & S. Paulo & 1881 & & \\
\hline Carlos Ferreira da Silva & $》$ & 1877 & & \\
\hline da Cunha Canto & $\gg$ & 1884 & & \\
\hline „Silva Chaves. & $》$ & 1888 & & \\
\hline de Almeida Magalhães Sobrinbo. & Minas Geraes & $\begin{array}{l}1000 \\
1875\end{array}$ & & \\
\hline "Andrade Pinto & Rio de Janeiro & 1856 & & \\
\hline "Campos Maia & 》 & 1891 & & \\
\hline * Lima Ramos & Bahia & 1896 & & \\
\hline$»$ Oliveira Cruz & S. Paulo & 1900 & & \\
\hline Ernesto da Gama Cerqueira & Minas Geraes & 1868 & & \\
\hline Fernandes Lima & Rio G. do Sul & 1881 & & \\
\hline Figueira de Aguiar & S. Paulo & 1881 & & \\
\hline Galvão de Souza Mello & $»$ & 1891 & & \\
\hline Gê Badaró. . & Minas Geraes & 1891 & & \\
\hline Gonçalves de Lima & Rio de Janeiro & 1869 & & \\
\hline José de Moura Filho. & Minas Geraes & 1858 & & \\
\hline \Manhães & Rio de Janeiro & 1890 & & \\
\hline Leite Ribeiro & $\gg$ & 1886 & & \\
\hline Martins Fontes & S. Paulo & $\begin{array}{l}1000 \\
1893\end{array}$ & & \\
\hline Meirelles Alves Moreira & Rio de Janeiro & 1867 & & \\
\hline Olympio Machado. & Babia & 1845 & & \\
\hline Paulo da Silva Prado & S. Paulo & 1881 & & \\
\hline Ribeiro Machado. . & Maranhão & 1889 & & \\
\hline Soulnier de Pierrelevée & "Nentand & $\begin{array}{l}1009 \\
1886\end{array}$ & & \\
\hline Teixeira de Carvalho Durão. & Rio de Janeiro & 1873 & & \\
\hline
\end{tabular}




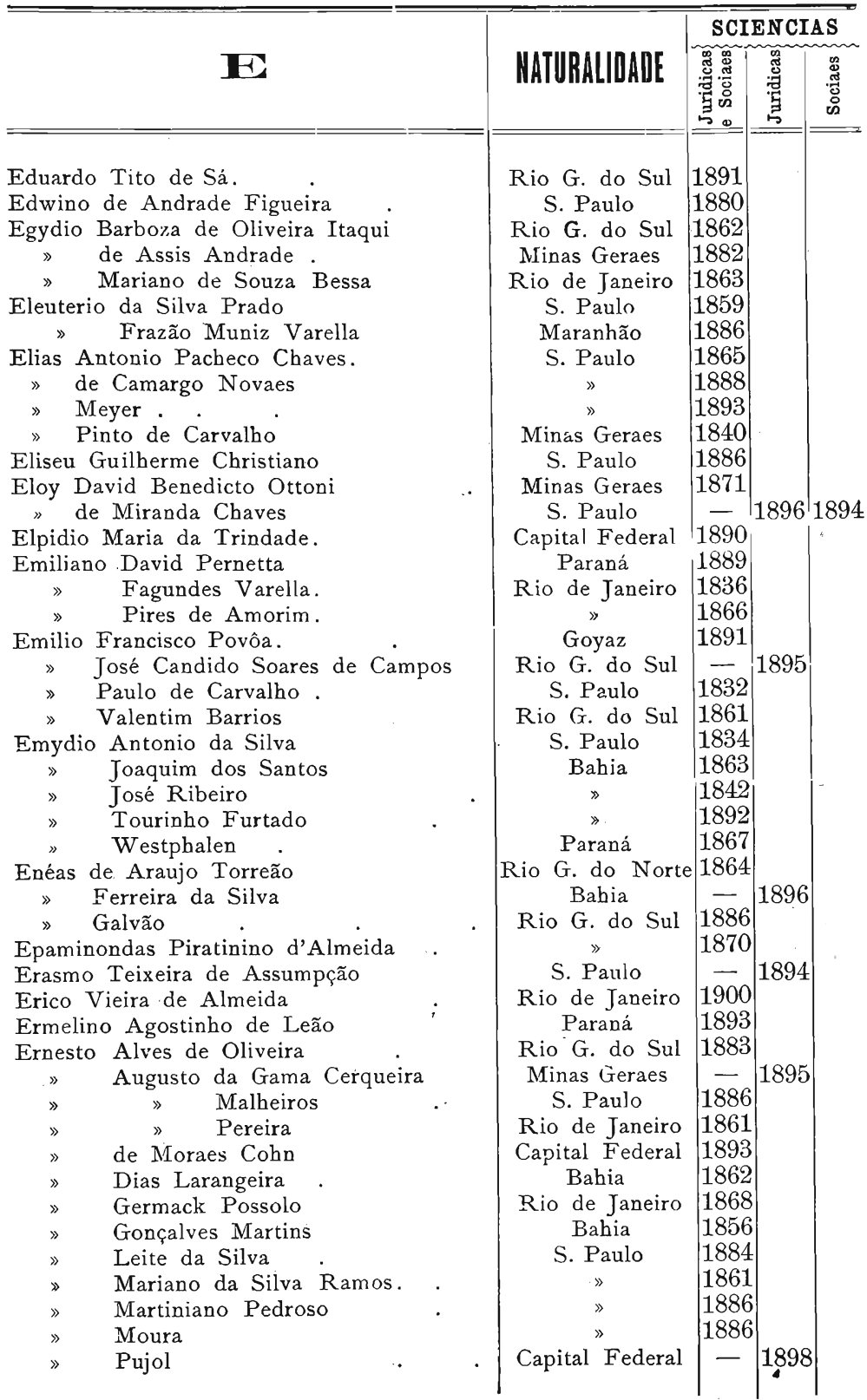




\begin{tabular}{|c|c|c|c|c|}
\hline & & SCI & IENC] & \\
\hline $\mathrm{H}$ & NATURALIDADE & 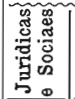 & 莺 & 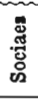 \\
\hline Ernesto Rodrigo Goulart Penteado & S. Paulo & 1891 & & \\
\hline " Rudge da Silva Ramos & $》$ & 1889 & & \\
\hline Esperidião Eloy de Barros Pimentel Junior & Alagôas & 1877 & & \\
\hline Esquilio Francisco do Amaral Góes & S. Paulo & 1863 & & \\
\hline Estacio Corrêa & Paraná & 1891 & & \\
\hline Estevam Augusto de Oliveira Filho Junior & Rio G. do Sul & 1883 & & \\
\hline$» \quad$ de Araujo Almeida & Rio de Janeiro & 1886 & & \\
\hline José de Siqueira Filho & $\gg$ & 1866 & & \\
\hline Leão Bourroul. & França & 1881 & & \\
\hline Leite de Magalbães Pinto & Minas Geraes & 1891 & & \\
\hline Lobo Leite Pereira & Rio de Janeiro & 1890 & & \\
\hline Ribeiro de Souza Rezende & $》$ & 1863 & & \\
\hline$\gg \quad$ Rezende & S. Paulo & 1835 & & \\
\hline Euclides Fausto de Souza & Côrte & 1886 & & \\
\hline I» Francisco de Moura & S. Paulo & 1863 & & \\
\hline Eudoxio de Figueiredo & Capital Federal & 1897 & & \\
\hline Eugenio Adriano de Moraes & $\gg$ & 1892 & & \\
\hline 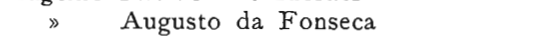 & S. Paulo & 1888 & & \\
\hline 》 Baptista de Oliveira & Londres & 1863 & & \\
\hline da Cunba Mello & Capital Federal & 1900 & & \\
\hline de Andrade Egas & S. Paulo & 1884 & & \\
\hline "Oliveira e Silva & $》$ & 1891 & & \\
\hline$»$ Paula Ferreira. & $»$ & 1872 & & \\
\hline »Valladão Catta Preta & Côrte & 1883 & & \\
\hline Ferreira da Cunba & $»$ & 1887 & & \\
\hline Gomes Pires Ferreira. & Capital Federal & 1891 & & \\
\hline Gonçalves Tourinho & Bahia & 1891 & & \\
\hline José Pereira de Mello & Rio de Taneiro & 1835 & & \\
\hline Lamartine de Andrade & Minas Geraes & 1892 & & \\
\hline Manoel de Toledo. & S. Paulo & 1863 & & \\
\hline Pinto Cardozo Malheiros & $»$ & 1861 & & \\
\hline » Teixeira Leite & Rio de Janeiro & 1875 & & \\
\hline Eurico Sergio Ferreira & Minas Geraes & 1891 & & \\
\hline Eurides Cunha & Paraná & 1894 & & \\
\hline Eusebio de Queiroz Mattozo Ribeiro & Rio de Janeiro & 1858 & & \\
\hline$»$ dos Passos Cardoso & Allemanha & 1887 & & \\
\hline Gomide Reichert & S. Paulo & 1889 & & \\
\hline $\begin{array}{c}\text { Innocencio Vaz Lobo da Camara } \\
\text { Leal }\end{array}$ & Paraná & 1879 & & \\
\hline Eusebio Silveira da Motta & ॥ & 1870 & & \\
\hline Euthiquio Carlos de Carvalho Gama & Alagôas & 1888 & & \\
\hline Evaristo de Araujo Cintra & S. Paulo & 1853 & & \\
\hline$» \quad »$ Oliveira. . & Minas Geraes & - & 1897 & \\
\hline Ferreira da Veiga & $»$ & 1855 & & \\
\hline$» \gg$ Gonzaga & Côrte & 1886 & & \\
\hline Gonçalves Marinho & Rio de Janeiro & 1876 & & \\
\hline Ladislau e Silva & Bahia & 1835 & & \\
\hline
\end{tabular}




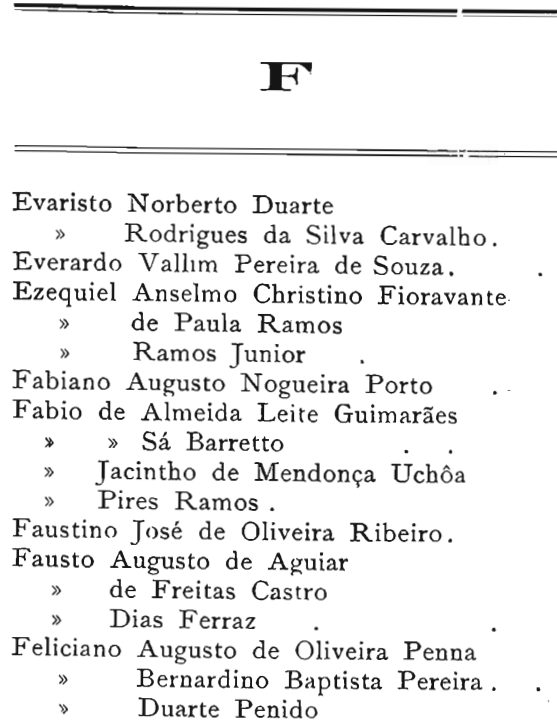

Felicio José de Miranda

" Ribeiro dos Santos Camargo

Felippe Antonio Gonçalves Junior.

» Corrêa Pinheiro e Silva.

» de Sampaio Corrêa.

» Gabriel de Castro Vasconcellos

» Ladeira de Faria

» Saboia Bandeira de Méllo.

* Xavier da Rocha .

Felisberto Barcellos Ferreira de Azevedo.

» Gomes Jardim

» Pereira da Silva

» Soares de Gouvêa Horta

Felix Bocayuva. .

» Generoso de Almeida e Silva José da Costa e Souza

$» \gg$ Serra.

¿ Pastana

» Xaviex da Cunha.

Felizardo Pinheiro de Campos.

$\gg$ » $\gg$ Muller

Fenelon da Silva Monte

Fernão de Souza Queiroz

Fernando Antonio de Barros

» Caldeira de Andrade.

» da Costa Leal Figueiredo

» de Siqueira Cardozo.

» Souza Barros .

» Ferraz de Andrade Junior

\begin{tabular}{|c|c|c|c|}
\hline \multirow[b]{2}{*}{ IATURALIDADE } & \multicolumn{3}{|c|}{ SCIENCIAS } \\
\hline & 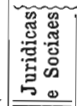 & 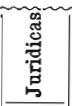 & $\begin{array}{l}\pi \\
0 \\
0 \\
0\end{array}$ \\
\hline Minas Geraes & 1862 & & \\
\hline Goyaz & 1868 & & \\
\hline S. Paulo & 1891 & & \\
\hline$\gg$ & 1861 & & \\
\hline$\gg$ & 1866 & & \\
\hline & & 1896 & \\
\hline S. Paulo & 1884 & & \\
\hline Rio de Janeiro & 1893 & & \\
\hline Sergipe & $1 \overline{1886}$ & 1895 & \\
\hline " & 1884 & & \\
\hline Bahia & 1869 & & \\
\hline Rio de Janeiro & 1839 & & \\
\hline Rio G. do Sul & 1865 & & \\
\hline Minas Geraes & $\overline{1869}$ & 1895 & 1892 \\
\hline Rio de Janeiro & 1866 & & \\
\hline Minas Geraes & 1881 & & \\
\hline$»$ & 1863 & & \\
\hline S. Paulo & 1863 & & \\
\hline Minas Geraes & 1890 & & \\
\hline S. Paulo & 1834: & & \\
\hline Rio de Janeiro & 1870 & & \\
\hline Minas Geraes & 1871 & & \\
\hline Babia & 1889 & & \\
\hline Ceará & 1886 & & \\
\hline Rio de Janeiro & 1855 & & \\
\hline Rio G. do Sul & 1887 & & \\
\hline Rio de Janeíro & 1855 & & \\
\hline Rio G. do Sul & 1855 & & \\
\hline Rio de Janeiro & 1863 & & \\
\hline Capital Federal & 1890 & & \\
\hline Minas Geraes & 1892 & & \\
\hline Rio de Janeiro & 1870 & & \\
\hline Sergipe & 1871 & & \\
\hline S. Paulo & 1900 & & \\
\hline Rio G. do Sul & 1854 & & \\
\hline Rio de Janeiro & 1834 & & \\
\hline$»$ & 1861 & & \\
\hline Sergipe & 1871 & & \\
\hline S. Paulo & 1886 & & \\
\hline Minas Geraes & 1862 & & \\
\hline Santa Catharina & 1892 & & \\
\hline Minas Geraes & 1863 & & \\
\hline S. Paulo & 1883 & & \\
\hline$»$ & 1882 & & \\
\hline$»$ & 1891 & & \\
\hline
\end{tabular}




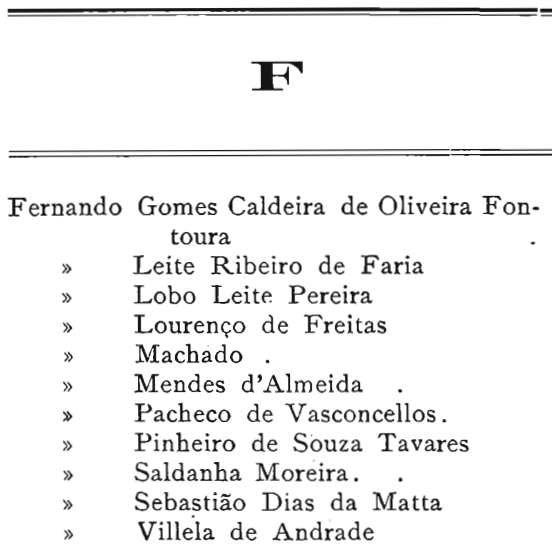

Fidelix de Oliveira

» Ignacio de Andrade Botelho

Filastrio Nunes Pires

Filinto Manoel Teixeira.

Firmiano de Moraes Pinto

Firmino Antonio da Silva Whitaker Filbo

» de Souza Lima

Estevão Pinheiro

$\gg$ Rodrigues Silva

Firmo d'Albuquerque Diniz

$»$ de Souza Vianna.

Flaminio Antonio do Nascimento Lessa

Flavio Augusto de Oliveira Queiroz

$»$ de Barros Franco

„ Salles Dias

» Farnezi da Paixão Junior

» Ferreira de Camargo

» Guedes de Araujo

Florencio Carlos de Abreu e Silva

Floriano Antonio de Moraes Junior

» de Souza Neves Junior

Leite de Assis.

Florindo Loureiro Sampaio.

Fortunato dos Santos Moreira

» José de Camargo Junior

» Luiz Barreto Filho.

„Raphael Nogueira Penido

Francisco Accaci Corrêa.

Accioli Lins

» Aguiar de Barros.

» Alvares da Silva Campos $\left(\mathbf{I}^{\circ}\right)$

$\gg \gg \gg\left(2 .^{\circ}\right)$

» $\gg$ d'Azevedo Macedo Junior

» Alvaro Bueno de Paiva

» Alves Branco.

\section{NATURALIDADE}

Minas Geraes

Rio de Janeiro

Minas Geraes

S. Paulo$$
»
$$

Maranhão

S. Paulo

Rio de Janeiro

Minas Greraes

Rio de Janeiro

S. Paulo

$$
\text { » }
$$

Minas Geraes

Santa Catharina

Maranbão

S. Paulo

$$
\text { » }
$$

Rio de Janeiro

Minas Geraes

Rio de Janeiro

Nio

$$
\gg
$$

S. Paulo

》.

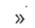

Minas Geraes

" Paulo

Bahia

Rio G. do Sul

S. Paulo

$$
\text { " }
$$

Rio de Janeiro

" Paulo

»

Rio G. do Sul

Minas Geraes

$$
\text { Pará }
$$

Pernambuco

S. Paulo

Minas Geraes

Rio de Janeiro "

Minas Geraes

Rio de Janeiro

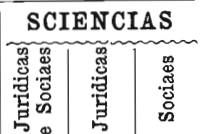

1835

1870

1876

1858

1895

1879

1878

1867

1886

1832

1889

1883

1856

1842

1891

1882

1886

1862

1870

1837

1852

1900

1843

1886

1898

1893

1856

1898

1883

1862

1892

1860

1878

1891

1886

1864

1889

1837

1864

1884

1862

1846

1884

1865

1983

1867 


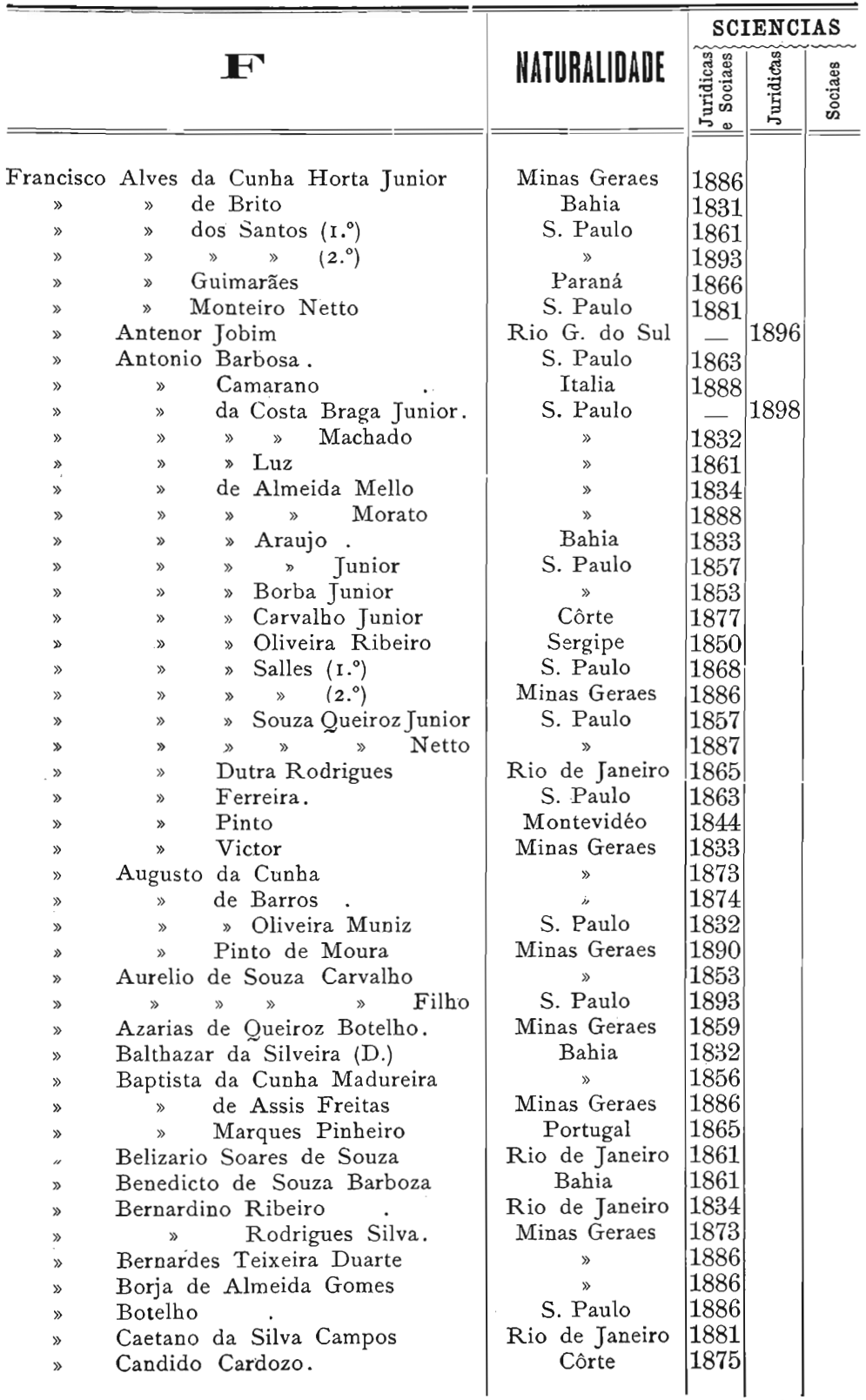




\begin{tabular}{|c|c|c|c|c|c|}
\hline & & & SCI & IENC & IAS \\
\hline & $\mathrm{H}^{\prime}$ & YATURALIDADE & 可嘀 & 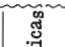 & 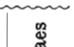 \\
\hline Francisco & Candido Gama Junior & Minas Geraes & 1891 & & \\
\hline$»$ & » de Bulhões Ribeiro & Rio de Janeiro & 1866 & & \\
\hline$»$ & Marciano da Fontoura & & & & \\
\hline & e Castro & Minas Geraes & 1835 & & \\
\hline$»$ & Cardozo de Araujo Ribeiro. & S. Paulo & - & 1896 & 1895 \\
\hline$》$ & Carlos de Araujo Brusque & Rio G. do Sul & 1845 & & \\
\hline$»$ & $\gg \quad \gg \quad$ & $\gg$ & 1882 & & \\
\hline$»$ & » Moreira & Minas Geraes & 1891 & & \\
\hline$»$ & $»$ dos Reis & Bahia & 1862 & & \\
\hline$»$ & » Mariani Junior & Pará & 1854 & & \\
\hline$»$ & Carneiro Monteiro Salles & Pernambuco & 1883 & & \\
\hline$\gg$ & » Ribeiro da Luz & Minas Geraes & 1881 & & \\
\hline$»$ & Cezario de Figueiredo Cortes J. ${ }^{\text {or }}$ & $»$ & 1879 & & \\
\hline » & Coelbo Borges . & Bahia & 1834 & & \\
\hline$»$ & » de Magalbães Junior & Rio de Janeiro & 1863 & & \\
\hline$»$ & » Duarte Badaró & Minas Geraes & 1883 & & \\
\hline$\gg$ & Cordeiro da Silva Guerra Filho. & S. Paulo & 1879 & & \\
\hline$»$ & Corrêa Borges . & $\gg$ & 1894 & & \\
\hline$》$ & $»$ Ferreira Rabello. & Minas Geraes & 1865 & & \\
\hline$»$ & Corsino de Assis. & $»$ & 1886 & & \\
\hline$»$ & da Costa Carvalho & Bahia & 1853 & & \\
\hline$»$ & '» „ Chaves Faria & Côrte & 1866 & & \\
\hline$»$ & » $»$ Guimarães & Rio de Janeiro & 1835 & & \\
\hline$》$ & "Cunha Brito & Paraná & 1888 & & \\
\hline$》$ & $»$ Silva Tavares & Rio G. do Sul & 1868 & & \\
\hline$»$ & das Chagas Alvares Fernandes & S. Paulo & 1834 & & \\
\hline$»$ & $» \quad » \quad$ Caminha. & , & 1835 & & \\
\hline » & de Araujo de Aragão Bulcão & Bahia & 1882 & & \\
\hline$»$ & "Assis Barcellos Corrêa & Minas Geraes & 1888 & & \\
\hline$»$ & $» \gg$ Barros Penteado & S. Paulo & 1884 & & \\
\hline$»$ & $» \quad »$ de Oliveira Braga & $»$ & 1860 & & \\
\hline$»$ & $» \gg \gg$ J $\gg$ Junior & $»$ & 1881 & & \\
\hline$»$ & » do Monte Carmello & $»$ & 1833 & & \\
\hline$»$ & $» \quad$ e Almeida & Matto Grosso & 1838 & & \\
\hline$»$ & » $\quad$ Lopes Mendes Ribeiro & $»$ & 1834 & & \\
\hline$»$ & $»$ Martins Costa & $»$ & 1862 & & \\
\hline$»$ & $» \quad » \quad$ Mascarenhas (D.) & Côrte & 1868 & & \\
\hline$»$ & $» \quad » \quad$ Pacheco Junior & S. Paulo & 1862 & & \\
\hline " & Netto. & $»$ & 1887 & & \\
\hline$\gg$ & $» \quad$ Peixoto Gomide & » & 1838 & & \\
\hline$»$ & $»$ Junior & $»$ & 1873 & & \\
\hline$»$ & $» \quad » \quad$ Pupo & $\gg$ & 1832 & & \\
\hline$\gg$ & $\gg \quad »$ Tavares. & Minas Geraes & 1870 & & \\
\hline$》$ & $» \quad$ Vieira Bueno & S. Paulo & 1841 & & \\
\hline 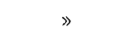 & » Borja de Macedo Couto. & Rio F. do Sul & 1890 & & \\
\hline$»$ & » Barros Lima Monte Razo & Minas Geraes & 1886 & & \\
\hline$»$ & »Campos Andrade Junior. & S. Paulo & 1886 & & \\
\hline
\end{tabular}




\begin{tabular}{|c|c|c|c|c|c|c|}
\hline & & & & SCI & IENCI & IAS \\
\hline & & $\mathrm{B}^{\circ}$ & NATURALIDADE & 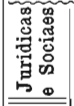 & 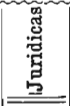 & 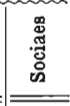 \\
\hline Francisco & de Campos & os Valladares & Rio de Janeiro & - & 1894 & 1795 \\
\hline$\gg$ & »Carvalh & ho Figueira de Mello. & Pernambuco & 1864 & & \\
\hline$»$ & $»$ & Prates & Rio G. do Sul & 1864 & & \\
\hline$»$ & »Castro & Junior & Capital Federal & 1900 & & \\
\hline$\gg$ & $» \quad »$ & Rodrigues Campos & Minas Geraes & 1895 & & \\
\hline$»$ & $\gg$ Faria V & Villas Bôas & Rio G. do Sul & 1874 & & \\
\hline$\gg$ & $\searrow$ Paula & Amaral. & Minas Geraes & 1886 & & \\
\hline$»$ & $» d$ & de Araujo Macedo. & S. Paulo & 1844 & & \\
\hline$»$ & $\gg \quad \gg$ & $» \quad » \quad$ Silva & Rio de Janeiro & 1866 & & \\
\hline » & $»$ & »Azevedo e Souza . & Rio G. do Sul & 1867 & & \\
\hline$»$ & $» \quad \mathrm{~d}$ & do Amaral Menna. & $\gg$ & 1867 & & \\
\hline$»$ & $» B$ & Belfort Duarte & Maranhão & 1864 & & \\
\hline$»$ & $» \mathrm{C}$ & Coelho Valmont & Minas Geraes & 1866 & & \\
\hline$»$ & $» \quad \mathrm{C}$ & $\begin{array}{c}\text { Cordeiro de Negreiros } \\
\text { Lobato }\end{array}$ & 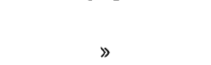 & 1870 & & \\
\hline$»$ & $»$ & da Fonseca Barros. & S. Paulo & 1891 & & \\
\hline$»$ & $\gg \mathrm{F}$ & Felicissimo & Minas Geraes & 1894 & & \\
\hline$»$ & $»$ & Fernandes Rabello & $\gg$ & 1867 & & \\
\hline$»$ & $\gg$ & Ferraz e Souza & Rio de Janeiro & 1866 & & \\
\hline$»$ & $»$ & Ferreira da Costa & Minas Geraes & 1861 & & \\
\hline$»$ & $»$ & » de Rezende & $»$ & 1855 & & \\
\hline$»$ & $»$ & Franco & S. Paulo & 1877 & & \\
\hline$»$ & $»$ & Guimarães & Minas Geraes & 1863 & & \\
\hline$»$ & $»$ & Leme & S. Paulo & 1861 & & \\
\hline$»$ & $\gg$ & Marinho & Rio de Janeiro & 1862 & & \\
\hline$»$ & $\gg N$ & Martins & S. Paulo & 1863 & & \\
\hline$»$ & $»$ & Monteiro de Barros Lima & Capital Federal & 1891 & & \\
\hline$»$ & $»$ & Moreira Barboza . & Minas Geraes & 1883 & & \\
\hline$»$ & $»$ & Negreiros Sayão Lobato & Rio de Janeiro & 1834 & & \\
\hline$»$ & $»$ & Oliveira Borges & S. Paulo & 1869 & & \\
\hline$»$ & $»$ & Paiva Baracho & $»$ & 1881 & & \\
\hline$》$ & $»$ & Pereira Barboza & $»$ & 1862 & & \\
\hline$»$ & $»$ & Pinto . & $»$ & 1886 & & \\
\hline$»$ & $\gg \mathrm{P}$ & Prestes Pimentel & Côrte & 1866 & & \\
\hline$»$ & $» \mathrm{R}$ & Rabello e Silva & S. Paulo & 1868 & & \\
\hline$»$ & $»$ & Ramos Horta Junior & Minas Geraes & 1861 & & \\
\hline$》$ & $»$ & Rodrigues Alves & S. Paulo & 1870 & & \\
\hline$»$ & $\gg \mathrm{R}$ & Rozo Ferraz & $»$ & 1850 & & \\
\hline$»$ & $\gg \mathrm{S}$ & Souza Filho & $»$ & 1850 & & \\
\hline$»$ & $»$ & Toledo & $»$ & 1858 & & \\
\hline$》$ & »Queiroz & $z$ Coutinho Mattozo & Loanda & 1834 & & \\
\hline$»$ & $»$ Sá Brit & ito Junior & Rio G. do Sul & 1832 & & \\
\hline$»$ & »Salles I & Dias Ribeiro & Minas Geraes & 1874 & & \\
\hline$»$ & $\gg \quad$ & Rosa & Rio de Janeiro & 1848 & & \\
\hline$»$ & $\bowtie$ Santa $E$ & Barbara Garcia & Goyaz & 1834 & & \\
\hline$»$ & $»$ Souza I & Martins & Piauby. & 1882 & & \\
\hline$»$ & $\gg \quad \gg 1$ & Ramos. & Minas Geraes & 1834 & & \\
\hline
\end{tabular}




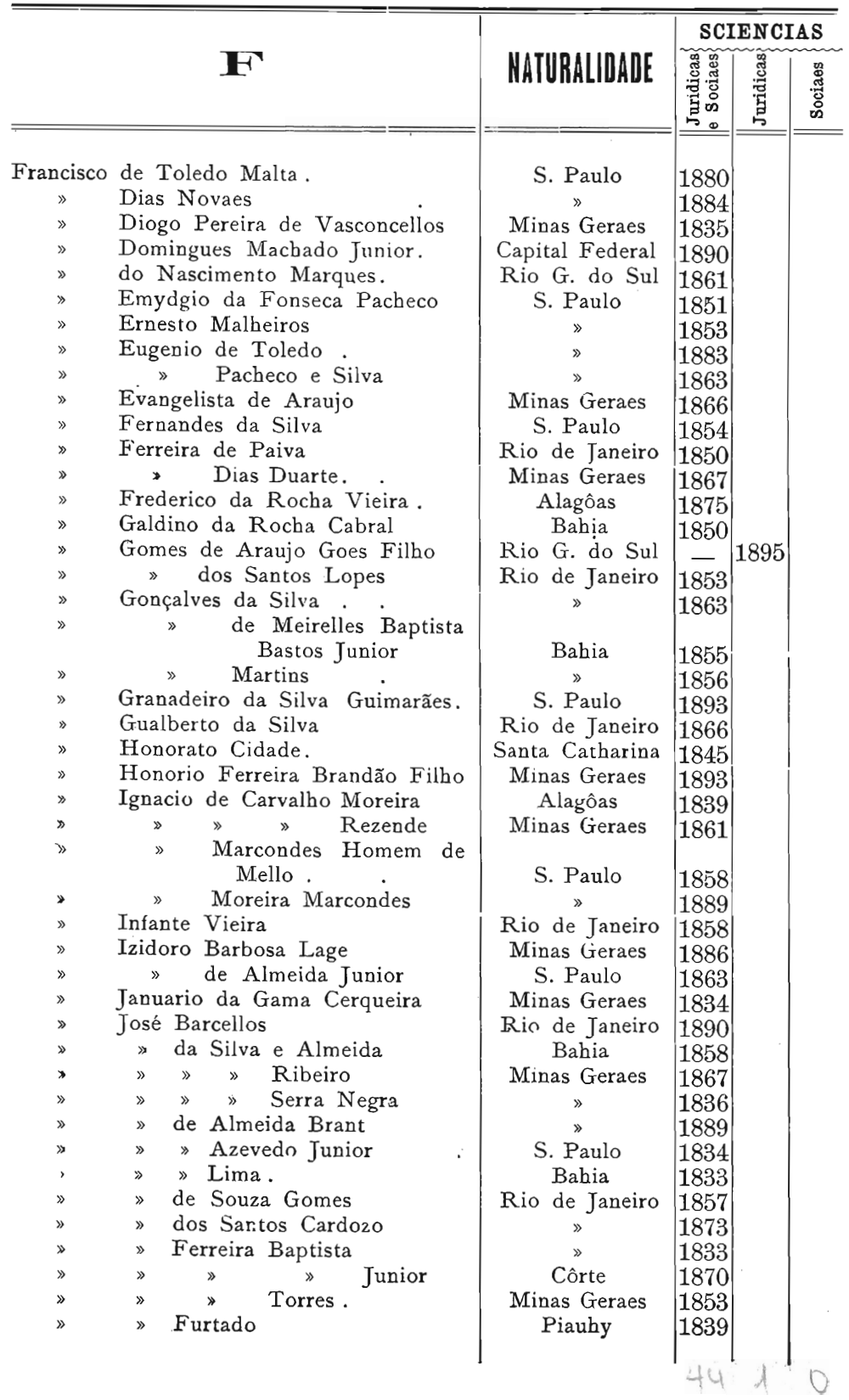




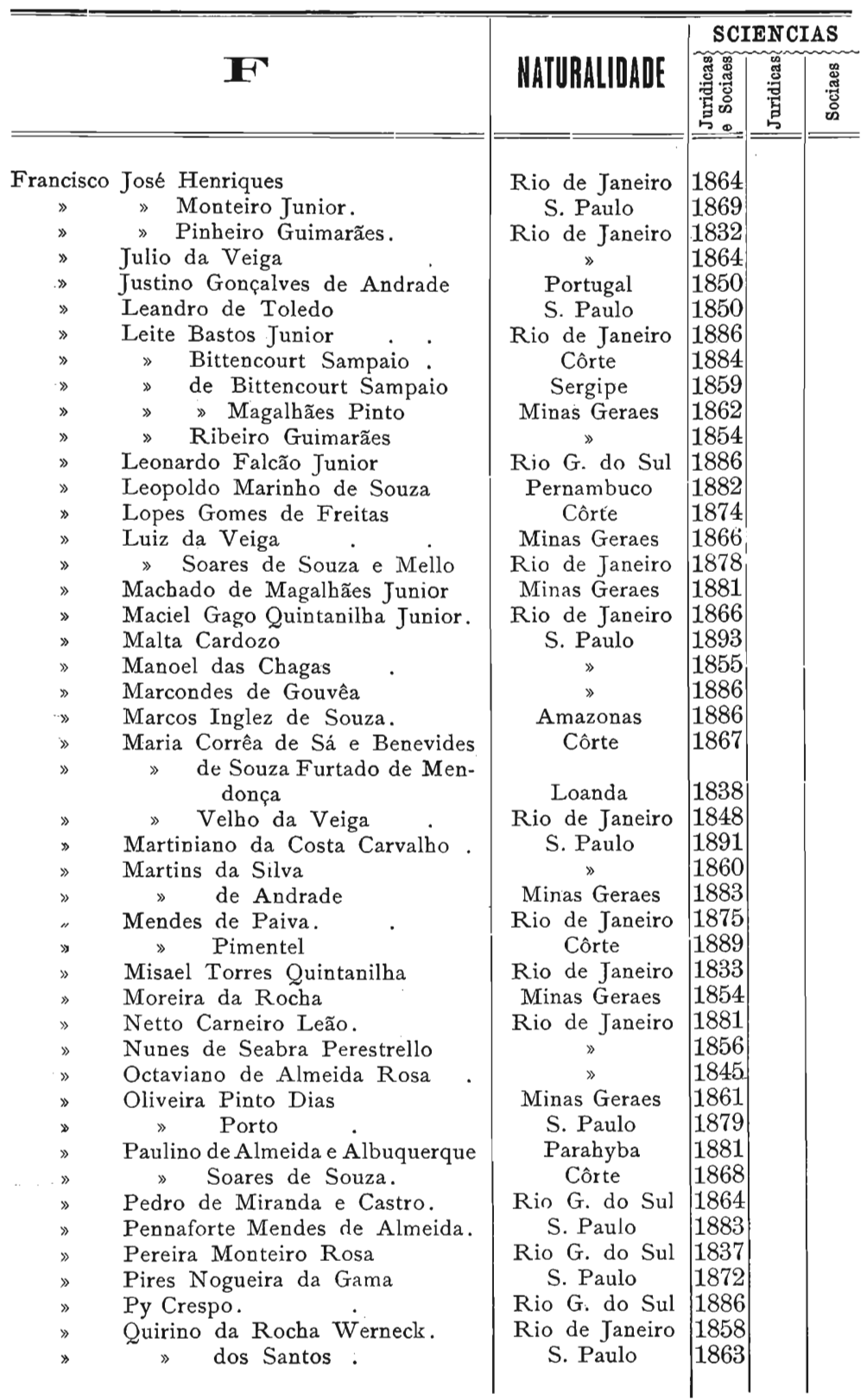




\begin{tabular}{|c|c|c|c|c|c|}
\hline & & & SCI & IENC & IAS \\
\hline & It & NATURALIDADE & 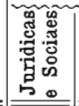 & 莺 & 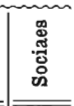 \\
\hline Francisco & Rangel Pestana & Rio de Janeiro & 1863 & & \\
\hline$»$ & Raphael de Araujo e Silva & S. Paulo & 1891 & & \\
\hline$»$ & Ribeiro da Silva Queiroz & Rio de Janeiro & 1833 & & \\
\hline$»$ & $»$ de Assis Rezende & Minas Geraes & 1893 & & \\
\hline$»$ & \# Azevedo Macedo & Pará & 1893 & & \\
\hline$»$ & Moura Escobar & S. Paulo & 1883 & & \\
\hline$»$ & »Teive e Argolo & Bahia & 1879 & & \\
\hline$»$ & d'Escobar & S. Paulo & 1852 & & \\
\hline$»$ & Soares Bernardes de Gouvêa & Minas Geraes & 1843 & & \\
\hline$»$ & $\gg$ Netto & $»$ & 1883 & & \\
\hline 》 & » Peixoto de Moura & $\gg$ & 1886 & & \\
\hline$»$ & Soter de Araujo Faria & S. Paulo & - & 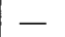 & 1894 \\
\hline$》$ & Teixeira de Souza Alves Junior. & Rio de Janeiro & 1862 & & \\
\hline$»$ & $» \quad$ Leite Guimarães & $\gg$ & 1886 & & \\
\hline$»$ & Thomaz de Carvalho. . & S. Paulo & 1886 & & \\
\hline$»$ & Torquato Fortes Junqueira & Minas Geraes & 1863 & & \\
\hline$»$ & Vieira Braga & Rio G. do Sul & 1856 & & \\
\hline$»$ & $» \quad$ da Costa. & Rio de Janeiro & 1838 & & \\
\hline$»$ & $» \quad$ de Almeida & $»$ & 1869 & & \\
\hline$»$ & $» \quad \gg$ Oliveira e Silva & S. Paulo & 1893 & & \\
\hline$»$ & Villela de Oliveira Marcondes & $»$ & 1881 & & \\
\hline$»$ & $\begin{array}{c}\text { Xavier da Costa Aguiar de An- } \\
\text { drada }\end{array}$ & $»$ & 1848 & & \\
\hline$\gg$ & $»$ Silva & Paraná & 1860 & & \\
\hline$\gg$ & $»$ de Barros & Goyaz & 1843 & & \\
\hline$»$ & $» \quad$ Carvalho : . & Bahia & 1886 & & \\
\hline$»$ & $» \quad »$ Paula Nogueira & S. Paulo & 1834 & & \\
\hline$»$ & $» \quad \gg$ Souza e Castro. & $»$ & 1886 & & \\
\hline$»$ & " Leite Pereira Lobo & $»$ & 1863 & & \\
\hline$»$ & » Moretz-Sobn . & Rio de Janeiro & 1863 & & \\
\hline$\gg$ & $\gg \quad$ Paes de Barros (I. $\left.{ }^{\circ}\right)$ & S. Paulo & 1854 & & \\
\hline$»$ & $\Rightarrow \gg \gg\left(2 .^{\circ}\right)$ & $»$ & 1884 & & \\
\hline$\gg$ & » Vahia Durão. . & Rio de Janeiro & 1853 & & \\
\hline Franklim & Gomes Souto. & Rio G. do Sul & 1865 & & \\
\hline$»$ & Washington da Silva e Almeida & Piauhy & 1867 & & \\
\hline Frederico & Augusto Alvares da Silva & Minas Geraes & 1852 & & \\
\hline$»$ & $» \quad$ Cleto Moreira & Rio de Janeiro & 1867 & & \\
\hline$»$ & de Almeida & Bahia & 1855 & & \\
\hline$»$. & Xavier de Brito & Rio de Janeiro & 1843 & & \\
\hline$\gg$ & Dabney de Avellar Brotero. & S. Paulo & 1860 & & \\
\hline$»$ & de Almeida Rego & Côrte & 1867 & & \\
\hline$»$ & „Barros Brotero & S. Paulo & - & 1896 & 1895 \\
\hline$»$ & do Nascimento Moura & Minas Geraes & 1868 & & \\
\hline$»$ & Ferreira França & Bahia & 1878 & & \\
\hline$»$ & José Cardozo de Araujo Abranches & S. Paulo & 1864 & & \\
\hline$\gg$ & Marcondes Machado & » & 1861 & & \\
\hline$»$ & Nunes de Seabra Perestrello & Rio de Janeiro & 1855 & & \\
\hline
\end{tabular}




\begin{tabular}{|c|c|c|c|c|}
\hline & & SCI & IENCI & IAS \\
\hline G & NATURALIDADE & 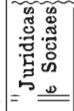 & 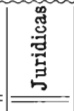 & 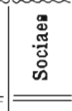 \\
\hline Frederico Vergueiro Steidel & S. Paulo & 1887 & & \\
\hline Frontino Ribeiro de Azevedo Vasconcellos & Rio de Janeiro & - & 1895 & \\
\hline Gabriel Caetano Guimarães Alvim. & Minas Geraes & 1858 & & \\
\hline$»$ de Oliveira Rocha & S. Paulo & 1891 & & \\
\hline$» \quad \gg \quad$ Santos. & Minas Geraes & 1881 & & \\
\hline » Paula Almeida Magalbães. & $»$ & 1855 & & \\
\hline »Vilbena Valladão & $»$ & 1898 & & \\
\hline » Dias da Silva Morewood & S. Paulo & 1882 & & \\
\hline » Diniz Junqueira. & Minas Geraes & 1837 & & \\
\hline Gomide . & S. Paulo & 1882 & & \\
\hline$\rightarrow$ José Rodrigues de Rezende & Minas Geraes & 1891 & & \\
\hline$\gg \# \quad \# \quad \operatorname{dos} \operatorname{Santos}\left(\mathrm{r}^{\circ}\right)$ & S. Paulo & 1836 & & \\
\hline$\gg \quad \gg \quad \gg \quad \gg \quad\left(2 .^{\circ}\right)$ & $»$ & 1866 & & \\
\hline Lessa & Minas Geraes & 1897 & & \\
\hline » Olinto de Carvalbo e Silva & S. Paulo & 1862 & & \\
\hline » Orlando Teixeira Junqueira & Minas Geraes & 1887 & & \\
\hline "Ozorio Mascarenhas & Rio G. do Sul & - & 1898 & 1897 \\
\hline Pinto de Almeida & Minas Geraes & 1859 & & \\
\hline > Pio da Silva & $»$ & 1859 & & \\
\hline$»$ de Loyolla. & & 1886 & & \\
\hline Ribeiro dos Santos. & S. Paulo & & 1895 & \\
\hline$\gg$ Villela de Andrade. & $»$ & 1889 & & \\
\hline Galdino de Freitas Travassos & Rio G. do Sul & 1866 & & \\
\hline Fernandes Pinbeiro & Rio de Janeiro & 1867 & & \\
\hline$» \quad$ Pedroso Bittencourt & S. Paulo & $|1892|$ & & \\
\hline$\because \quad$ Siqueira. & & & 1895 & \\
\hline Galeno Martins de Almeida & Rio de Janeiro & 1893 & & \\
\hline Gaspar Menna Barreto de Barros Falcão & Pernambuco & 1886 & & \\
\hline$» \quad$ Silveira Martins. . . . & Rio G. do Sul & 1855 & & \\
\hline Gastão Aldano Vaz Lobo da Camara Leal & S. Paulo & 1891 & & \\
\hline$» \quad$ da Cunha & Minas Geraes & 1884 & & \\
\hline " de Souza Mesquita & S. Paulo & 1884 & & \\
\hline » Galbardo Madeira & $»$ & 1892 & & \\
\hline Generoso Alves Ribeiro & Matto Grosso & 1861 & & \\
\hline » Marques dos Santos & Paraná & 1865 & & \\
\hline Genipro da Cunba d'Eça e Costa & Pernambuco & 1836 & & \\
\hline Genuino Antonio da Silva Peres & Rio de Janeiro & 1834 & & \\
\hline » Firmino Vidal Capistrano & Santa Catharina & 1873 & & \\
\hline Gentil Nelatan de Maura Rangel & Minas Geraes & 1893 & & \\
\hline Geraldino da Silva Campista & 》 & 1886 & & \\
\hline Geraldo Leite de Magalbães Gomes & $»$ & 1886 & & \\
\hline » Nogueira da Gama Carneiro Belens & Côrte & 1866 & & \\
\hline Germano Martins França & S. Paulo & - & 1895 & \\
\hline Gil Diniz Goulart & Rio de Janeiro & 1867 & & \\
\hline$»$ Pedro Yereirà da Silva. . & Minas Geraes & 1887 & & \\
\hline Gonçalo de Lagos Fernandes Bastos & Ceará & 1865 & & \\
\hline Graciano Alves de Azambuja & Rio G. do Sul & 1866 & & \\
\hline
\end{tabular}




\begin{tabular}{|c|c|c|c|c|}
\hline & & $\mathrm{SCI}$ & IENCI & IAS \\
\hline & NATIRALIDADE & 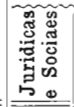 & 咆 & 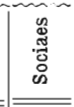 \\
\hline Gregorio Francisco de Miranda & Rio de Janeiro & 1876 & & \\
\hline Jrsé de Oliveira Costa Junior & S. Paulo & 1862 & & \\
\hline Tavares Maciel da Costa. & Cayena & 1837 & & \\
\hline Guido Cardoso de Menezes e Souza & Capital Federal & 1893 & & \\
\hline Saraiva Nogueira & Rio de Janeiro & 1861 & & \\
\hline Guilherme Bandeira de Gouvêa & $»$ & 1833 & & \\
\hline : $\quad$ Caetano da Silva Filbo. & S. Paulo & 1874 & & \\
\hline de Almeida Migalbães & Minas Geraes & 1858 & & \\
\hline Jósé Montenegro & S. Paulo & 1868 & & \\
\hline Gustavo Adolpho e Castro. & $»$ & 1857 & & \\
\hline$» \quad$ Sucbow & Rio de Janeiro & 1863 & & \\
\hline $\begin{array}{l}\text { Alberto de Aquino e Castro. } \\
\text { Galvão }\end{array}$ & $\begin{array}{l}\text { Goyaz } \\
\text { Rio G. do Sul }\end{array}$ & $\begin{array}{l}1880 \\
1884\end{array} \mid$ & & \\
\hline » Julio Pinto Pacca & S. Paulo & 1887 & & \\
\hline » Marcondes de Albuquerque & Paraná & 1868 & & \\
\hline Heitor Frederico Gambara & Italia & 1895 & & \\
\hline$»$ Teixeira Penteado & S. Paulo & 1900 & & \\
\hline Heliodoro Delfim da Silva & Rio de Janeiro & 1868 & & \\
\hline Henrique Amancio de Souza Jordão & $»$ & 1891 & & \\
\hline Antonio Alves de Carvalho. & Côrte & 1866 & & \\
\hline » Barnabé Vincent & França & 1876 & & \\
\hline Bawden & Minas Geraes & - & 1898 & \\
\hline Borges Monteiro & Côrte & 1889 & & \\
\hline Cappellano & Montevidéo & $|1897|$ & & \\
\hline de Almeida Valgas & Santa Catharina & 1891 & & \\
\hline Francisco de Avila & Rio G. do Sul & 1855 & & \\
\hline Graça. & Ceará & 1880 & & \\
\hline João Dodsworth & Córte & 1868 & & \\
\hline José Coelbo & Capital Federal & 1893 & & \\
\hline$»$ Rodrigues & Rio de Janeiro & 1891 & & \\
\hline Teixeira & $\gg$ & 1865 & & \\
\hline Ladislau da Silva Araujo & $»$ & 1863 & & \\
\hline Lascasas & S. Paulo & 1883 & & \\
\hline Limpo de Abreu. & Rio de Janeiro & 1861 & & \\
\hline I,obato Marcondes Machado. & S. Paulo & - & 1896 & 1895 \\
\hline Marques de Carvalbo & Rio de Janeiro & 1866 & & \\
\hline Martins Chaves & Rio G. do Sul & 1883 & & \\
\hline Porchat de Assis. & S. Paulo & 1869 & & \\
\hline Proost de Camargo & $»$ & 1891 & & \\
\hline Herculano Augusto de Padua e Castro & Côrte & 1875 & & \\
\hline " Chrispim de Carralho & S. Paulo & 1890 & & \\
\hline de Figueiredo e Souza & Rio de Janeiro & 1866 & & \\
\hline Galdino de Alvarenga & S. Paulo & 1888 & & \\
\hline Marcos Ingles de Souza & Pará & 1876 & & \\
\hline Nina Parga & Maranbão & - & 1894 & \\
\hline Ribeiro : & Minas Geraes & 1894 & & \\
\hline Hermano Cardozo da Silva Ramos & Côrte & 1867 & & \\
\hline
\end{tabular}




\begin{tabular}{|c|c|c|c|c|}
\hline & & SCI & ENCI & IAS \\
\hline T & NATURALIDALE & 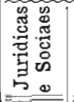 & 惫 & 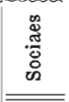 \\
\hline Hermenegildo Lopes àe Moraes & Goyaz. & 1891 & & \\
\hline » Militão de Almeida. & Pará & 1881 & & \\
\hline Rodrigues de Barros & Minas Geraes & 1886 & & \\
\hline Hermogeneo Pereira de Queiroz e Silva & Córte & 1868 & & \\
\hline Hermogenes Francisco de Aguilar Pantoja & Rio de Janeiro & 1842 & & \\
\hline » Martiniano Mendes Pereira & Ceará & 1865 & & \\
\hline Herodiano Alipio Camboim & Rio G. do Sul & - & 1895 & \\
\hline Hilario Gomes Nogueira & Minas Geraes & 1841 & & \\
\hline$» \quad "$ de Castro & S. Paulo & 1855 & & \\
\hline Honorio Augusto Ribeiro & Minás Geraes & 1860 & & \\
\hline de Souza Pacheco. & Rio de Janeiro & 1891 & & \\
\hline Hermeto Carneiro Leão & Minas Geraes & 1853 & & \\
\hline$» \quad$ Pinto de Figueiredo & $\gg$ & 1874: & & \\
\hline Rodrigues de Faria e Castro & $"$ & 1837 & & \\
\hline » Teixeira Coimbra & Rin) de Janeiro & 1865 & & \\
\hline Horacio Andrade & Minas Geraes & 1887 & & \\
\hline de Magalhães Gomes & $»$ & 1890 & & \\
\hline Gonçalves Pereira & S Paulo & 1900 & & \\
\hline Leão Belfort Sabino & Santa Catharina & 1889 & & \\
\hline Moreira Guimarães & Côrte: & 1880 & & \\
\hline Ribeiro da Silva. & Minas Geraes & 1891 & & \\
\hline Hygino Alves de Abreu e Silva & $»$ & 185 & & \\
\hline \Chaves de Camargo & Rio (i. do Sul & - & 1895 & 1894 \\
\hline Hyppolito Cabeda & $»$ & 1884 & & \\
\hline de Camargo: & S. Paulo & 1872 & & \\
\hline José de Araujo. & $\gg$ & 1863 & & \\
\hline "Soares de Souza & Maranhão & 1842 & & \\
\hline$» \gg \gg$ Filbo & S. Paulo & 1866 & & \\
\hline Ladislau Alves Cruz & $»$ & 1881 & & \\
\hline Pacheco Alves de Araujo & Paraná & 1889 & & \\
\hline Ibrahim Carneiro da Cruz Machado & Minas Geraes & 1887 & & \\
\hline Ignacio Alves Pereira & Rio G. do Sul & 1868 & & \\
\hline Antonio de Assis Monteiro & Minas Geraes & 1862 & & \\
\hline de Loyolla Gomes da Silva & $»$ & 1867 & & \\
\hline "Mendonça Uchôa & Alagôas & 1879 & & \\
\hline "Queiroz Lacerda & S. Paulo & 1882 & & \\
\hline Francisco Silveira da Motta & Goya: & 1838 & & \\
\hline Joaquim Barboza Junior & Rio de Janeiro & 1844 & & \\
\hline » de Paiva Freire de Andrade & Rio G. do Sul & 1836 & & \\
\hline José de Araujo. & Rio de Janeiro & 1833 & & \\
\hline Manoel Alves de Azeredo & $»$ & 1833 & & \\
\hline Maranbão da Rocha Vieira & Alagôas & 1882 & & \\
\hline Marcondes Romeiro & S. Paulo & 1879 & & \\
\hline Moreno Bodrigues Chaves. & Rio G. do Sul & 1893 & & \\
\hline Ribeiro de Assis & Minas Geraes & 1890 & & \\
\hline Rodrigues Bernardes $\left(P .{ }^{e}\right)$ & Espirito Santo & 1839 & & \\
\hline Soares de Bulbões Jardim. & Goyaz & 1875 & & \\
\hline
\end{tabular}




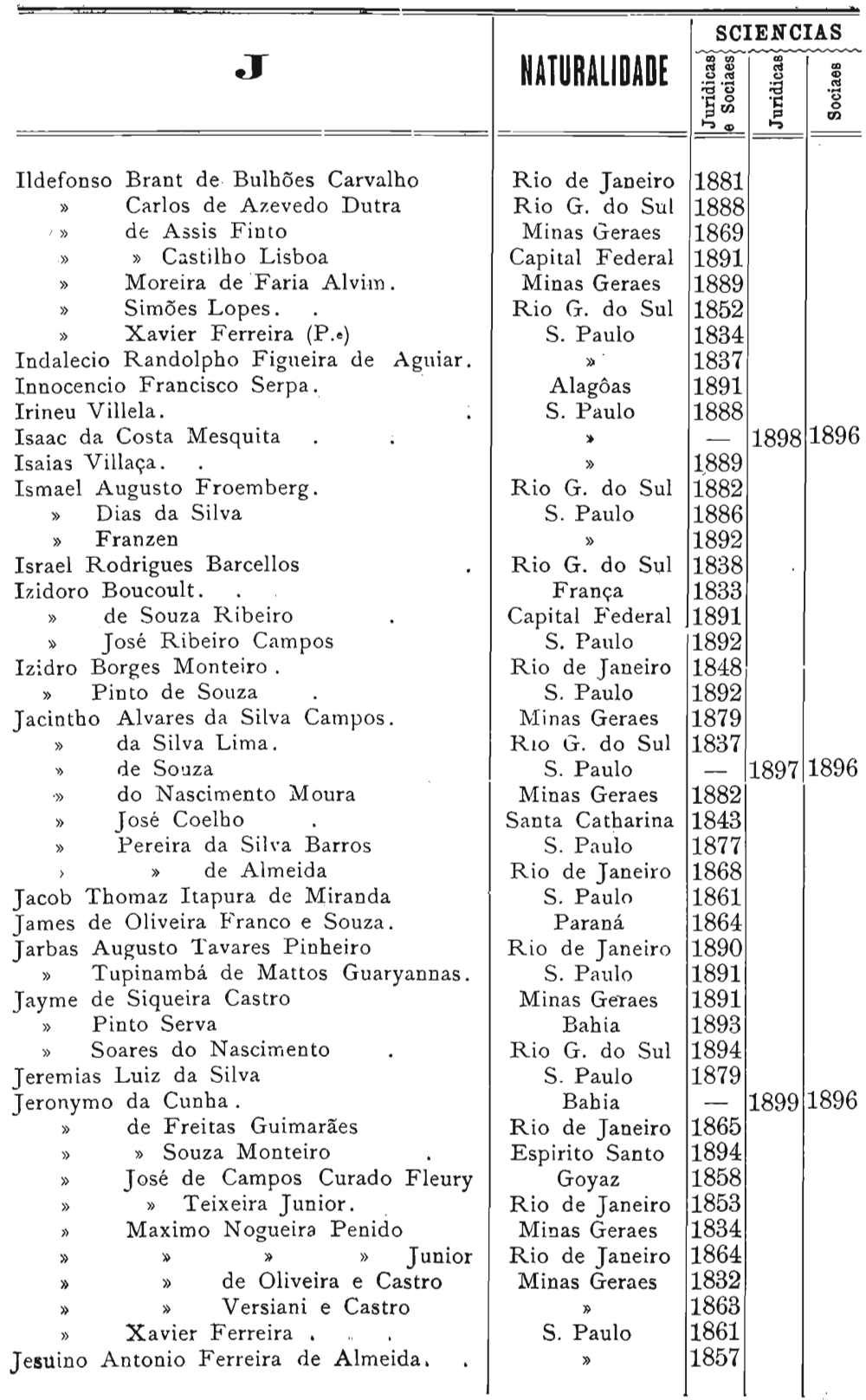




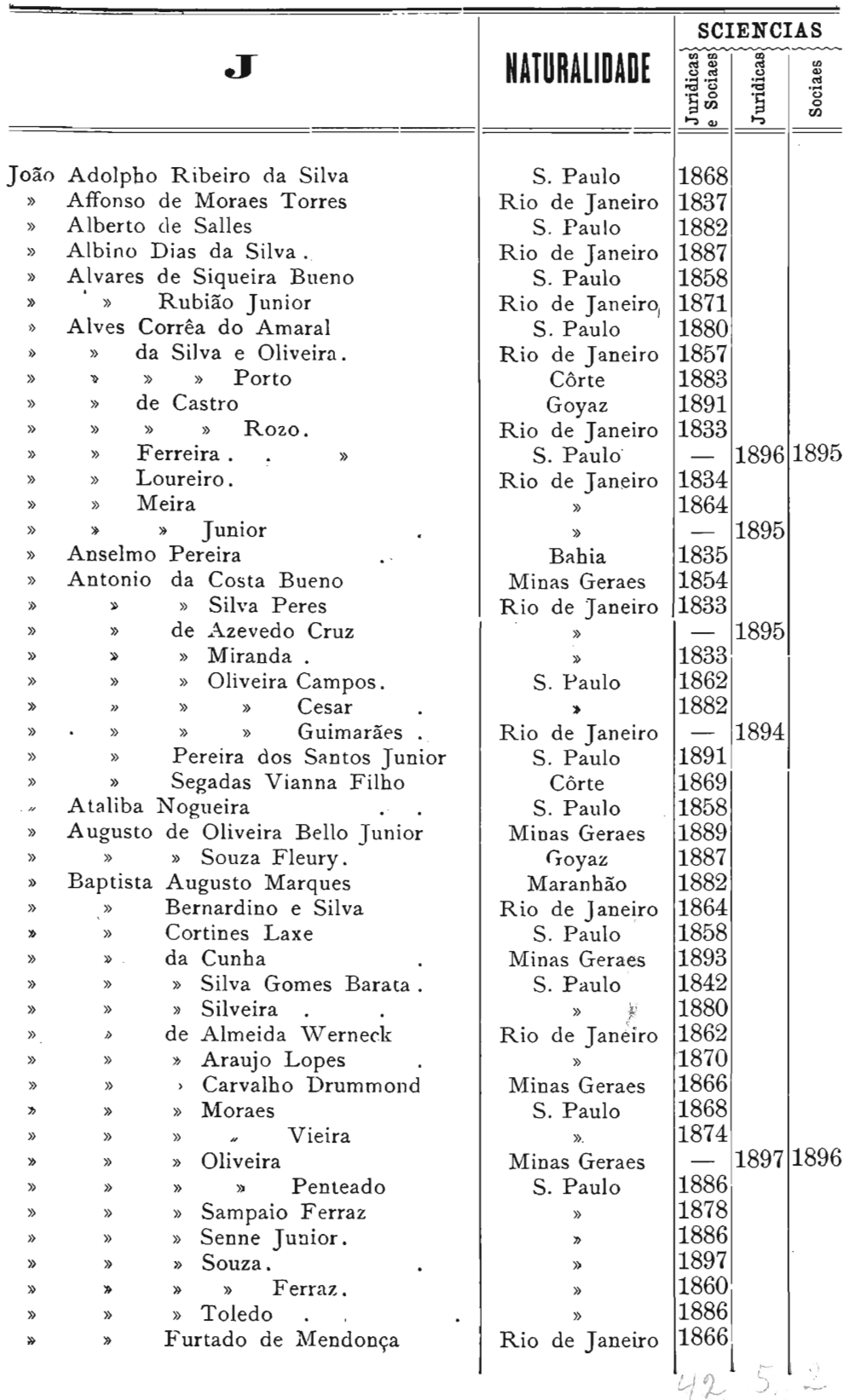




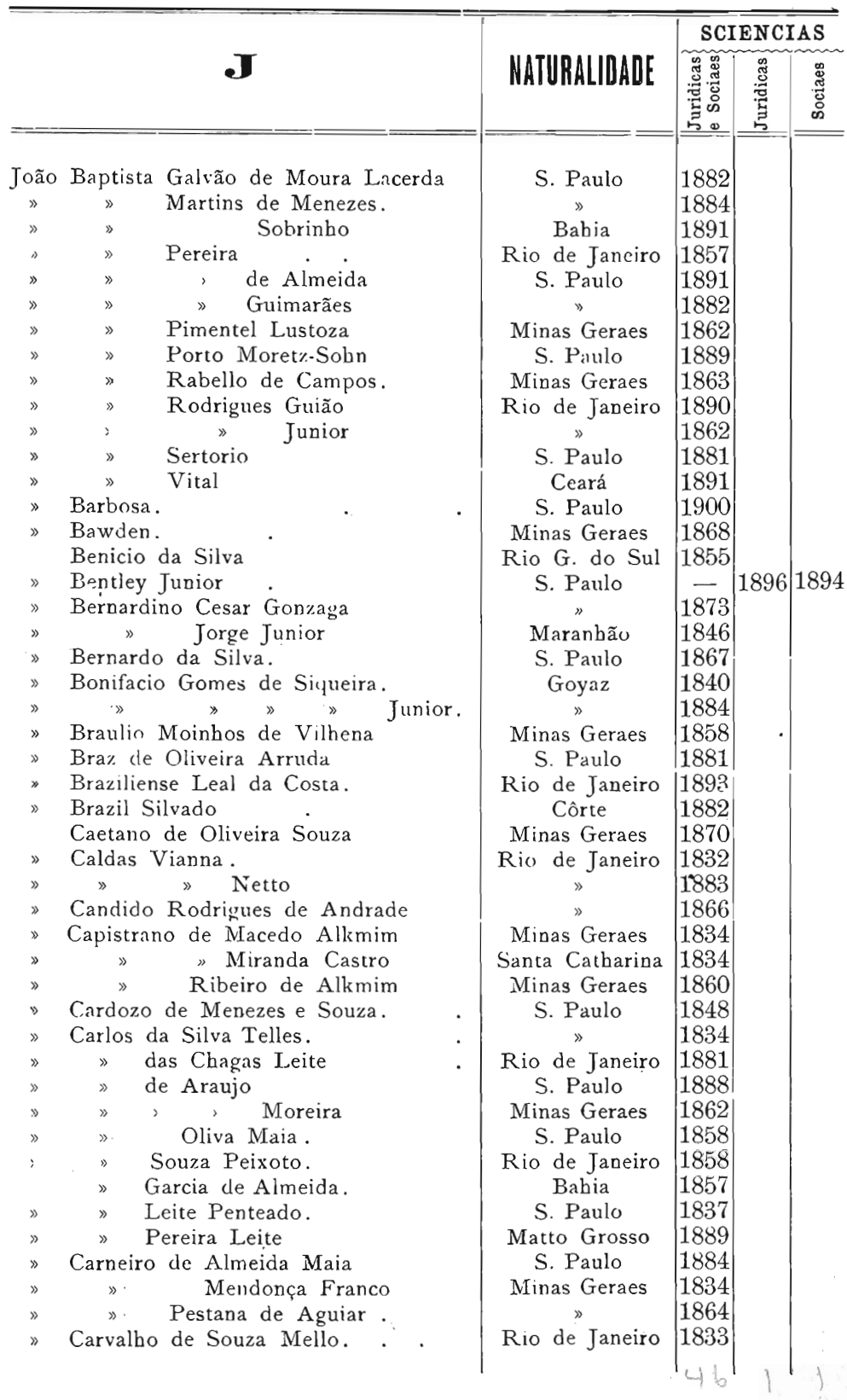




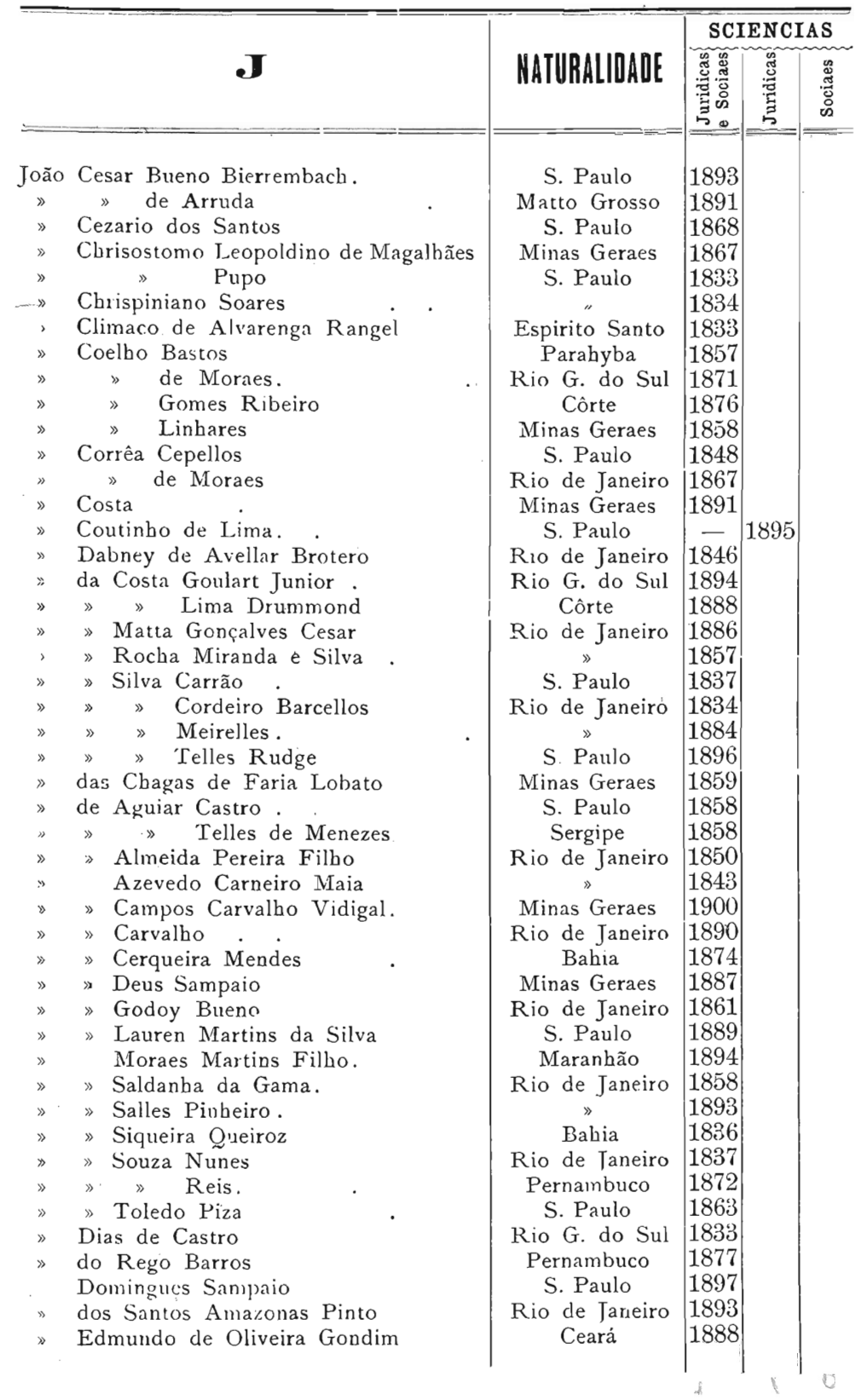




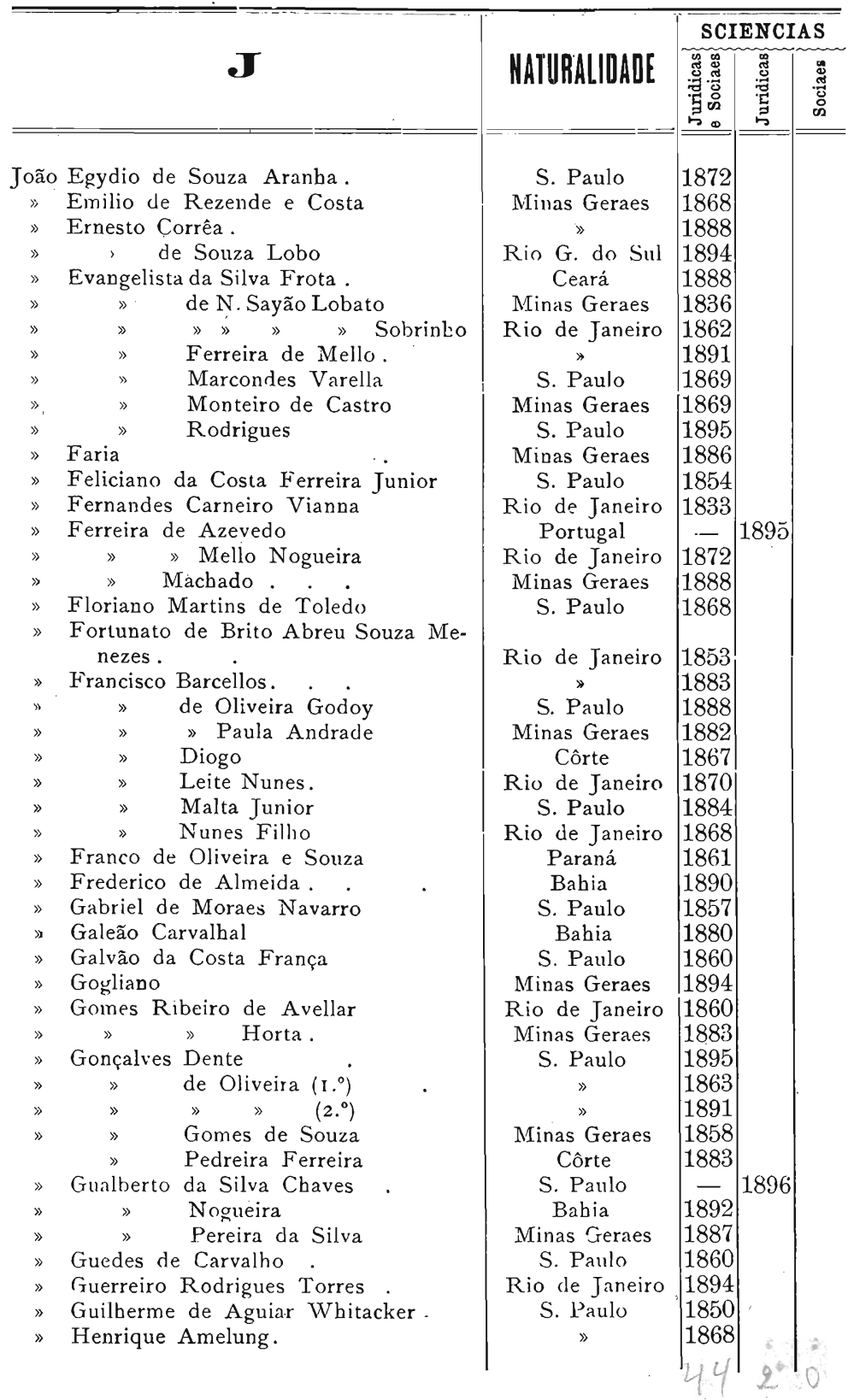




\begin{tabular}{|c|c|c|c|c|c|c|}
\hline & \multirow[b]{2}{*}{ MATURALIDADE } & \multicolumn{3}{|c|}{ SCIENCIAS } \\
\hline & & & & 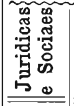 & 莺 & 恋 \\
\hline João & Hippolito das Mercês & & Pará & 1900 & & \\
\hline$\gg$ & Honorio de Magalhães Gomes ( & (P.e) & Minas Geraes & 1839 & & \\
\hline$»$ & Ignacio da Cunha & & Rio de Janeiro & 1866 & & \\
\hline.$\varnothing$ & » Silveira da Motta. & & Bahia & 1848 & & \\
\hline$»$ & Teixeira & & Rio G. do Sul & 1867 & & \\
\hline$»$ & Jacintho de Mendonça. & & $»$ & 1882 & & \\
\hline$»$ & $\gg \quad » \quad$ Junior & $\cdot$ & $»$ & 1881 & & \\
\hline 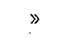 & Gonçalves de Andrade & $\left(\mathrm{P} .{ }^{\circ}\right)$ & Portugal & 1864 & & \\
\hline » & Jorge de Siqueira Franco & & S. Paulo & - & 1895 & 1896 \\
\hline$»$ & José Cardozo Junior & & Rio de Janeiro & 1832 & & \\
\hline$»$ & » Carneiro da Silva. & & » & 1863 & & \\
\hline$»$ & $»$ Coutinho . . & & $»$ & 1832 & & \\
\hline$\gg$ & » de Almeida Couto & & Bahia & 1835 & & \\
\hline » & ». Andrade Bastos & & Rio de Janeiro & 1871 & & \\
\hline$»$ & $» \quad » \quad$ Pinto Junior & & $\gg$ & 1846 & & \\
\hline$»$ & $» \quad$ Araujo & & Minas Geraes & 1886 & & \\
\hline$\gg$ & $» \quad$ Azevedo & & S. Paulo & - & 1898 & \\
\hline$"$ & » Vieira Junior. & & Rio de Janeiro & 1889 & & \\
\hline$»$ & » Frederico Ludovice Filho & & S. Paulo & 1874 & & \\
\hline$»$ & "Gomes da Silva & & Rio de Janeiro & 1866 & & \\
\hline$»$ & $»$ Pereira & & Minas Geraes & 1833 & & \\
\hline$»$ & $» \quad » \quad$ Bastos Junior. & & Rio de Janeiro & 1860 & & \\
\hline$»$ & $»$ Pedrosa & . & Paraná & 1865 & & \\
\hline$\Rightarrow$ & » Rodrigues. & & S. Paulo & 1845 & & \\
\hline$\gg$ & Kopke & & Rio de Janeiro & 1875 & & \\
\hline$»$ & Lenie da Silva. & . & S. Paulo & 1868 & & \\
\hline$»$ & $\begin{array}{l}\text { Lins Vieira e Cansanção de Si } \\
\text { Junior }\end{array}$ & inimbú & Rio G. do Sul & 1878 & & \\
\hline$»$ & Lopes da Silva Coito & & Rio de Janeiro & 1832 & & \\
\hline$»$ & Luiz Alves Junior & & Minas Geraes & 1889 & & \\
\hline$»$ & $»$ d'Avila & & Rio de Janeiro & 1854 & & \\
\hline$»$ & » de Mattos Pereira de Cas & tro & $»$ & 1855 & & \\
\hline$\gg$ & » Soares Martins & & Bahia & 1856 & & \\
\hline$»$ & Manoel Carlos de Gusmão & & Rio de Janeiro & 1879 & & \\
\hline$»$ & $» \quad$ de Almeida Barboza & & S. Paulo & 1833 & & \\
\hline$\gg$ & Dima e Silva & & Rio de Janeiro & 1861 & & \\
\hline$»$ & Lopes de Carvalho Pim & nentel. & Bahia & 1833 & & \\
\hline$\gg$ & Marcellino de Souza Gonzaga. & & Rio de Janeiro & 1841 & & \\
\hline , & Marciano Oliveira da Silva & & Côrte & 1886 & & \\
\hline$»$ & Marcondes de Moura Romeiro & & S. Paulo & 1865 & & \\
\hline$»$ & » dos Santos. & & Côrte & 1886 & & \\
\hline$»$ & Maria da Costa . . & & Rio de Janeiro & 1890 & & \\
\hline$»$ & » de Miranda Mansos & & Minas Geraes & $-\overline{-}$ & 1894 & 1895 \\
\hline$»$ & » Moraes Junior & & Pará & 1864 & & \\
\hline$\gg$ & do Valle. & • & Côrte & 1884 & & \\
\hline » & $» \quad$ Lisboa Junior . & . & Rio de Janeiro & 1868 & & \\
\hline$»$ & Martins de Carvalho Mourão & . : & Minas Geraes & 1892 & & \\
\hline
\end{tabular}




\begin{tabular}{|c|c|c|c|c|c|}
\hline & & & SCI & LENCI & IAS \\
\hline & ] & NATURALIDADE & 赑 & శ్లై & $\stackrel{\Xi}{\sharp}$ \\
\hline João & Martins de Mello Junior & S. Paulo & - & 1895 & \\
\hline & $»$ França. & Rin G. do Sul & 1862 & & \\
\hline$»$ & Mauricio de Sampaio Vianna & Bahia & 1891 & & \\
\hline & Mendes de Almeida & Maranhão & 1853 & & \\
\hline & $» \quad » \quad$ Junior & S. Paulo & 1877 & & \\
\hline$»$ & Miguel de Mello Taques & $»$ & 1838 & & \\
\hline$\gg$ & Muniz Cordeiro Tatagiba & Bahia & 1847 & & \\
\hline$»$ & Monteiro da Cunha Salgado & S. Paulo & 1884 & & \\
\hline$»$ & $\gg \quad » \operatorname{Luz}$ & Rio de Janeiro & 1863 & & \\
\hline$\gg$ & Nepomuceno de Souza Freire & S. Paulo & 1849 & & \\
\hline$»$ & Freire Junior & $»$ & 1886 & & \\
\hline$»$ & Nogueira da Motta. & $»$ & 1884 & & \\
\hline$»$ & Nunes Ramalbo Junior & $»$ & 1861 & & \\
\hline$\gg$ & Olavo'Eloy de Andrade & Minas Geraes & 1886 & & \\
\hline$»$ & Pamphilo Vellosa de Assumpção & Paraná & 1889 & & \\
\hline$\gg$ & Passos & S. Paulo & 1881 & & \\
\hline$»$ & Paulo Barbosa Lima & Ceará & 1886 & & \\
\hline$»$ & de Almeida Couto & Bahia & -1 & 1895 & \\
\hline$»$ & » $\gg \quad$ Magalhães . & Rio de Janeiro & 1854 & & \\
\hline$»$ & $»$ dos Santos Barreto Filho & $\gg$ & 1857 & & \\
\hline$»$ & » Martinho Lebfeld & Allemanha & 1896 & & \\
\hline$»$ & Pedro Belfort Vieira & Maranhão & 1868 & & \\
\hline$»$ & » Carvalbo de Moraes & Rio de Janeiro & 1853 & & \\
\hline 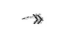 & » da Veiga Filho & Minas Geraes & 1886 & & \\
\hline$»$ & » Dias Vieira. & Maranbão & 1841 & & \\
\hline$»$ & » Moretz-Sohn & Minas Geraes & 1869 & & \\
\hline$\gg$ & Peregrino Viriato de Medeiros & Ceará & 1874 & & \\
\hline 》 & Pereira Corsino & S. Paulo & 1891 & & \\
\hline$»$ & » da Silva Borges Fortes & Rio G. do Sul & 1869 & & \\
\hline$»$ & $» \gg$ Continentino. & Côrte & 1878 & & \\
\hline$»$ & » Monteiro Junior $\left(\mathrm{I} .^{\circ}\right)$. & $\gg$ & 1872 & & \\
\hline$»$ & $\gg \gg \quad\left(2 .^{\circ}\right)$. & S. Paulo & - & 1895 & 1894 \\
\hline$»$ & Pinheiro da Silva & Minas Geraes & 1887 & & \\
\hline$»$ & Pinto Borba . . & S. Paulo & 1858 & & \\
\hline$»$ & $»$ da Silveira Coelho & Rio de Janeiro & 1864 & & \\
\hline$»$ & $»$ de Castro & S. Paulo & 1866 & & \\
\hline$»$ & » Moreira & Minas Geraes & 1859 & & \\
\hline$"$ & $» \quad$ Nunes Junior & S. Paulo & 1863 & & \\
\hline$»$ & Piragyba . : & Rio de Janeiro & 1872 & & \\
\hline$»$ & Pires da Silva Junior. & $»$ & 1852 & & \\
\hline$\gg$ & » Nogueira da Gama & S. Paulo & 1870 & & \\
\hline$»$ & Propicio Azambuja d'Avila & Rio G. do Sul & 1894 & & \\
\hline$»$ & Py Crespo & $»$ & 1888 & & \\
\hline$»$ & Quirino do Nascimento & S. Paulo & 1864 & & \\
\hline$»$ & Raphael de Azevedo & Rio de Janeiro & 1864 & & \\
\hline$»$ & Ribeiro da Silva & S. Paulo & 1858 & & \\
\hline$»$ & $»$ de Moura Escobar & $»$ & 1893 & & \\
\hline
\end{tabular}




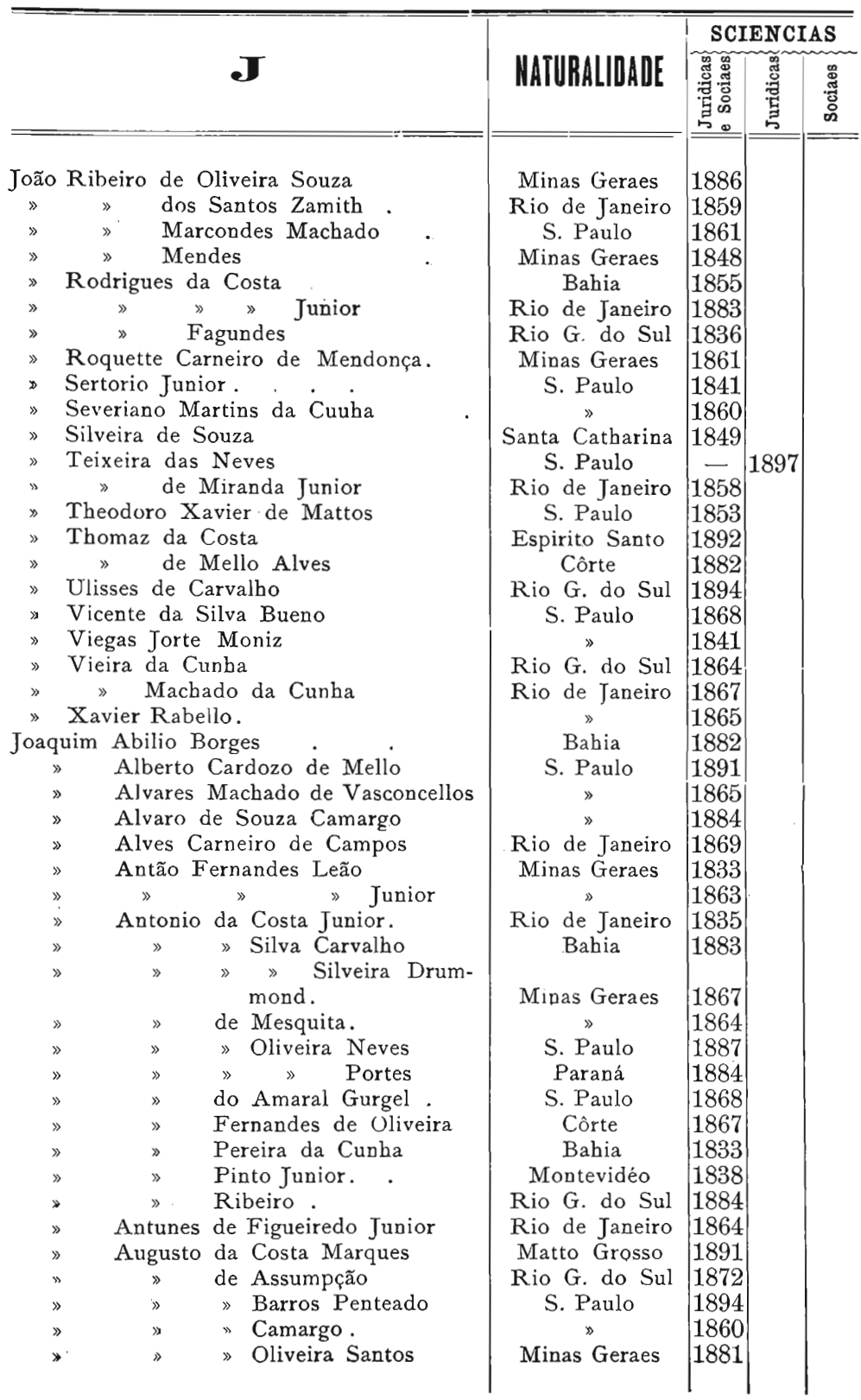




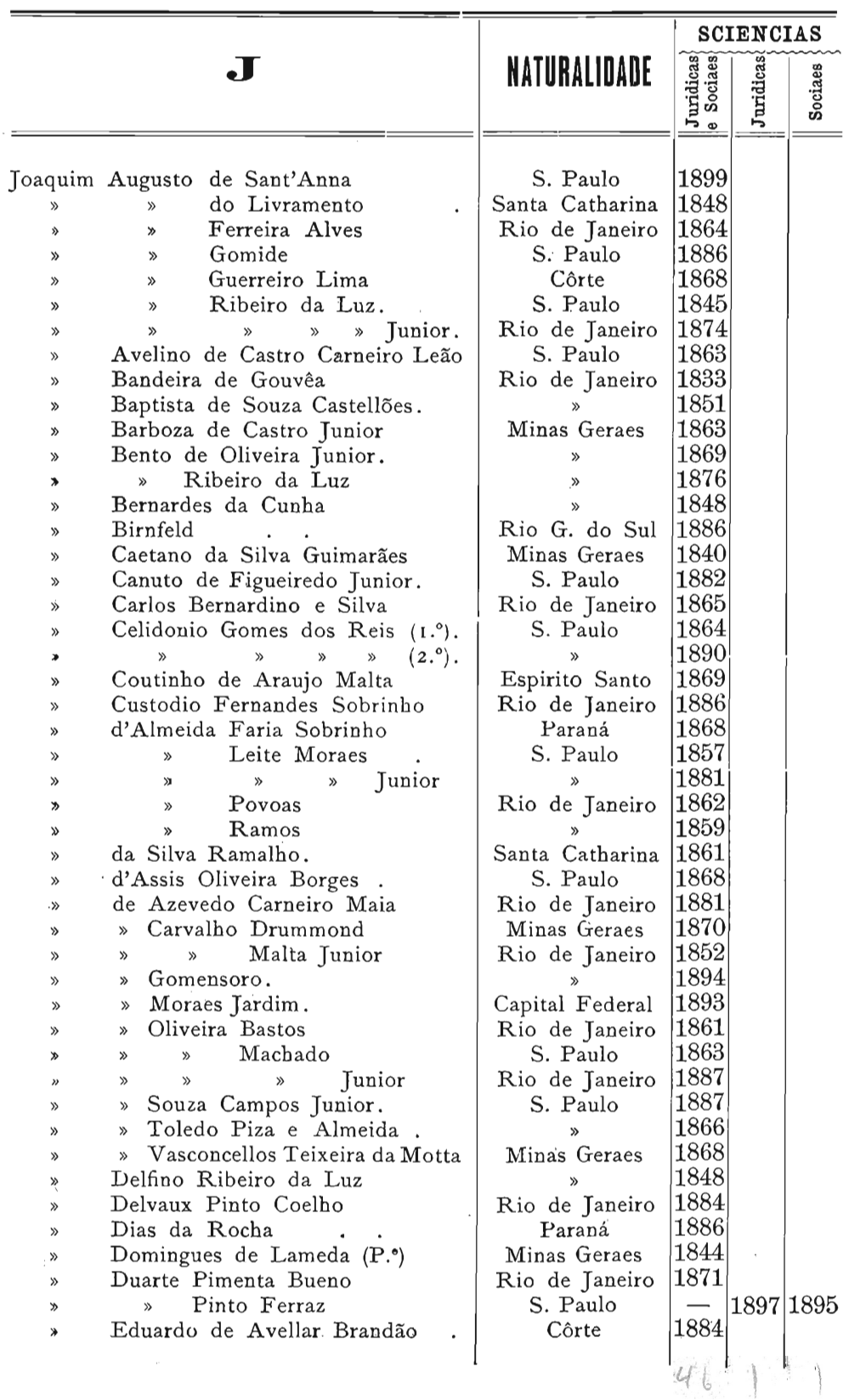




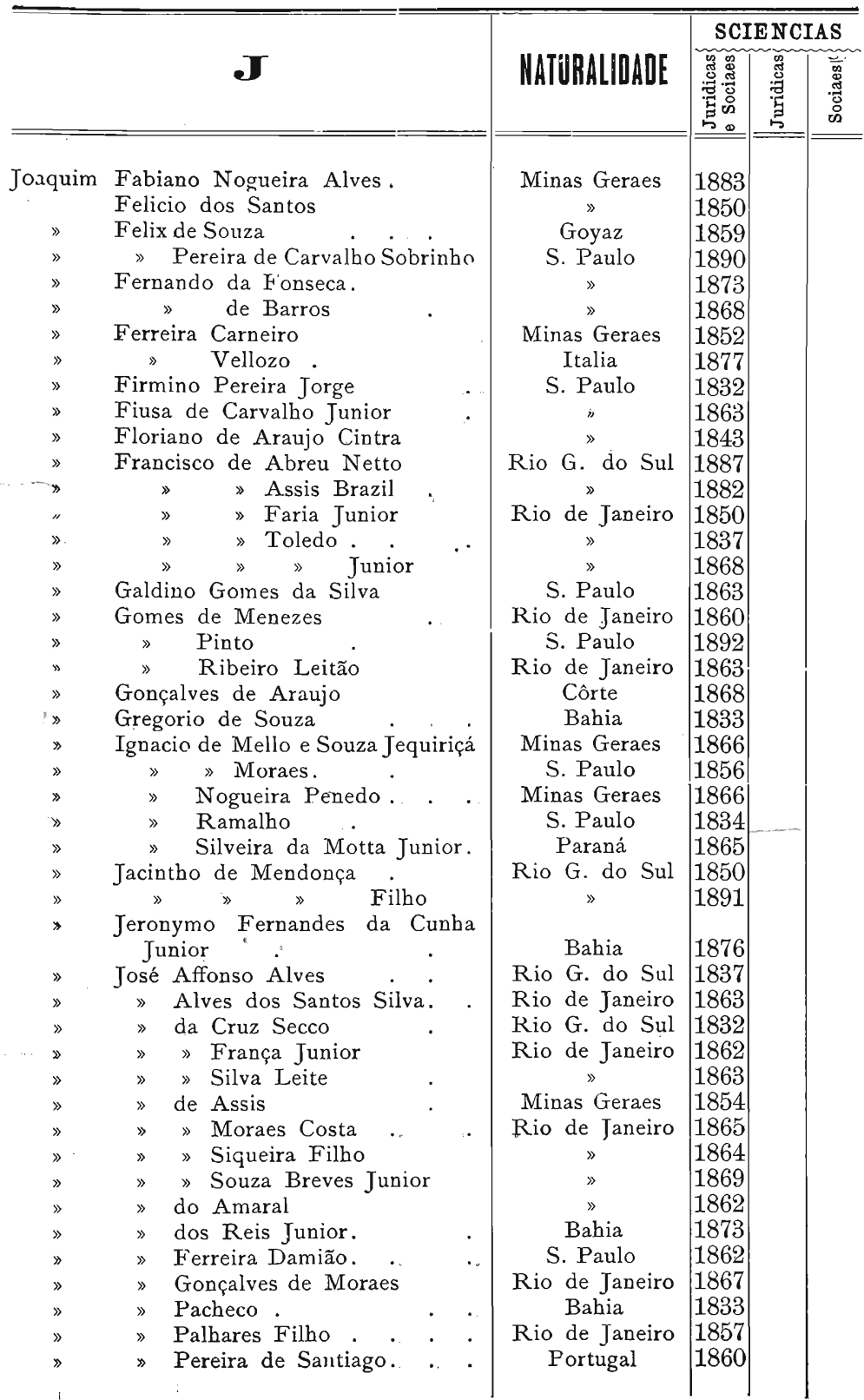




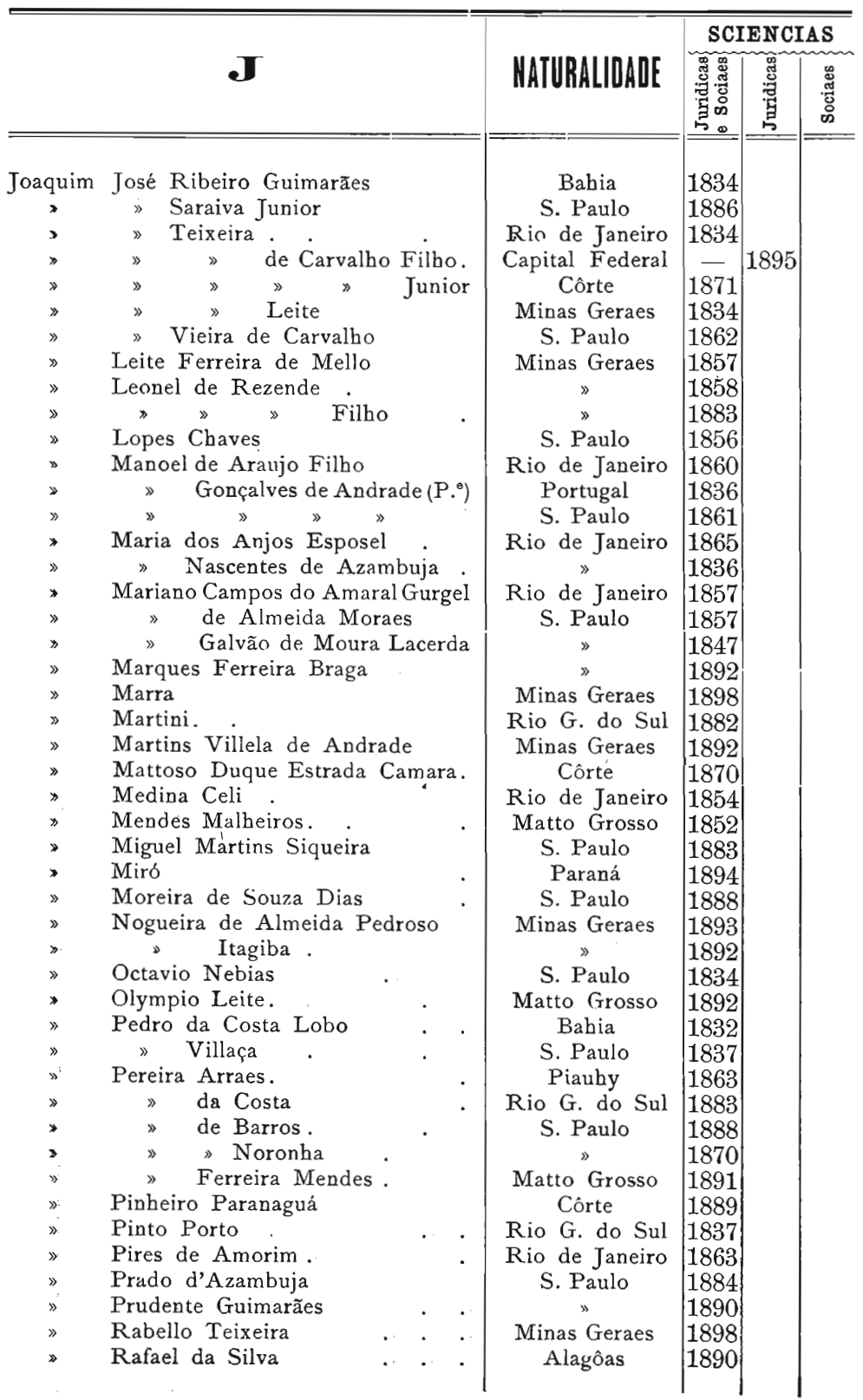




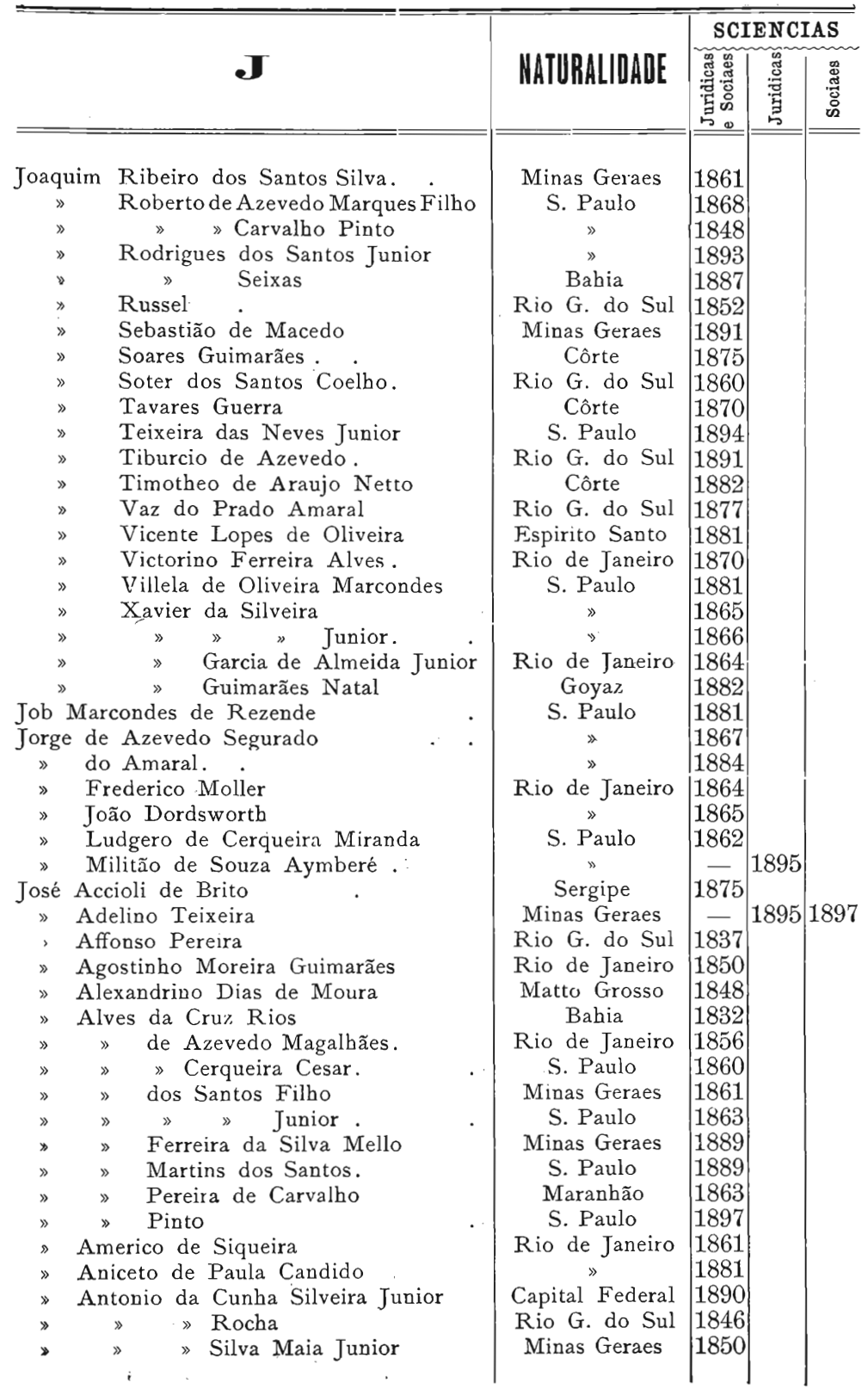




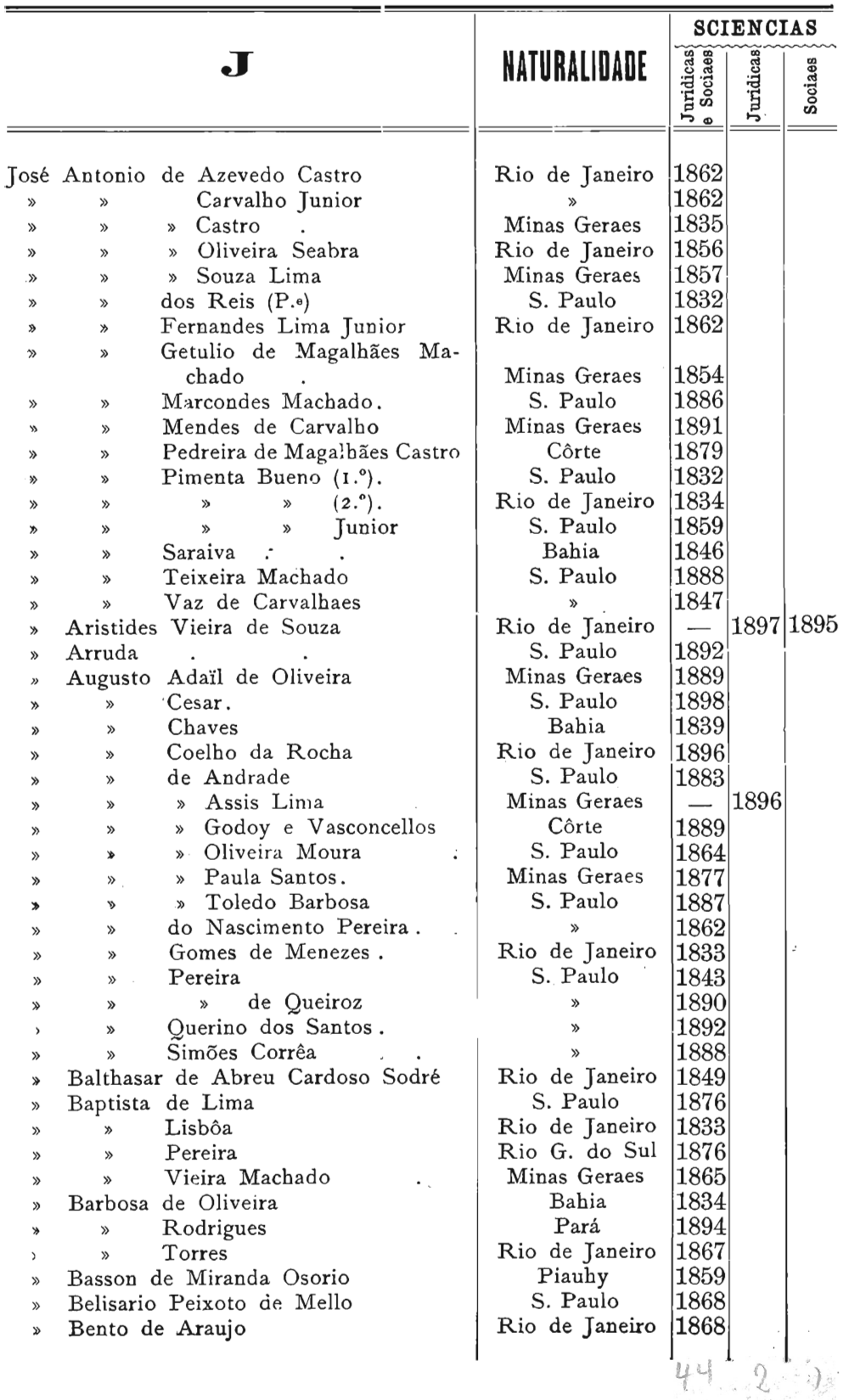




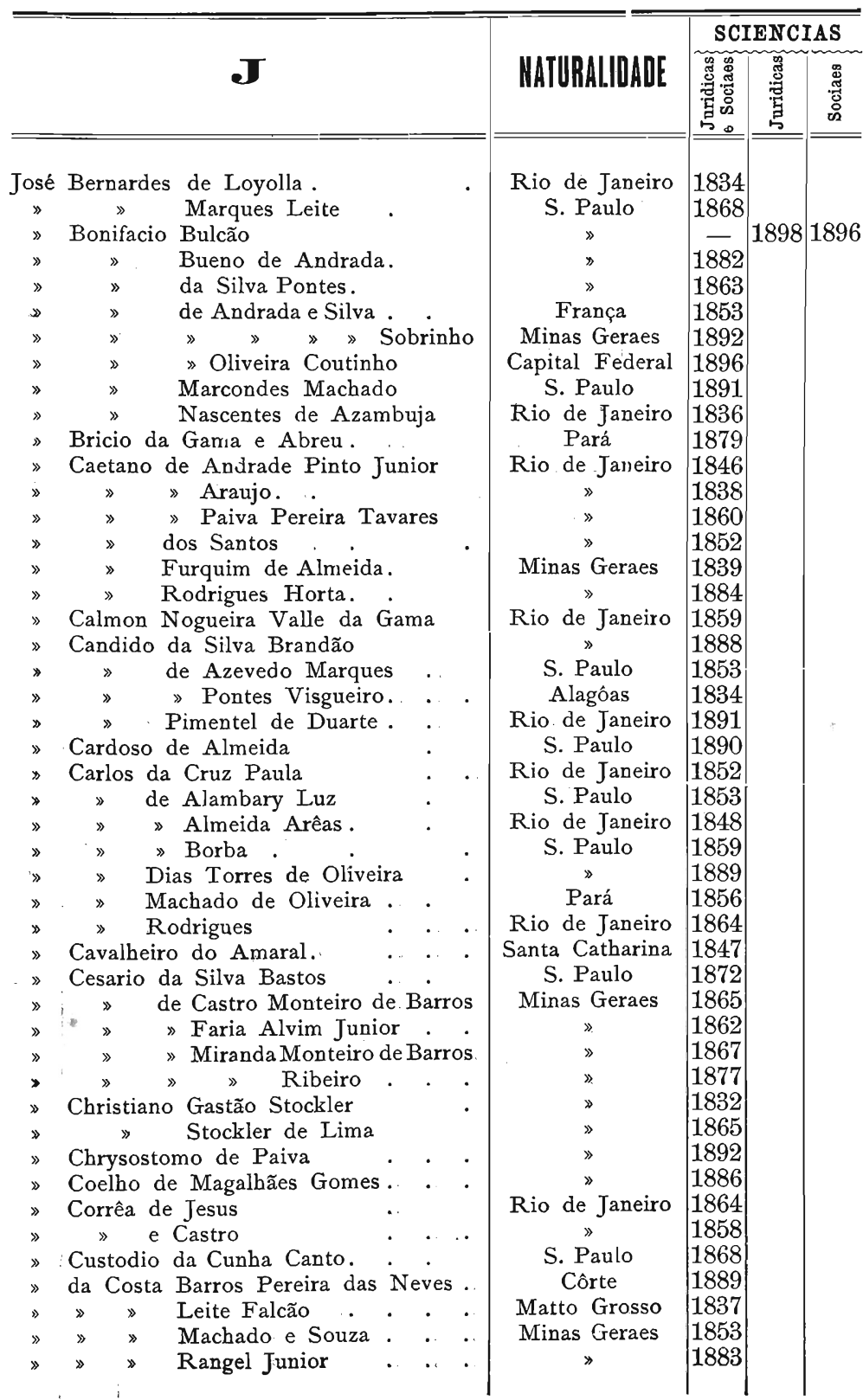




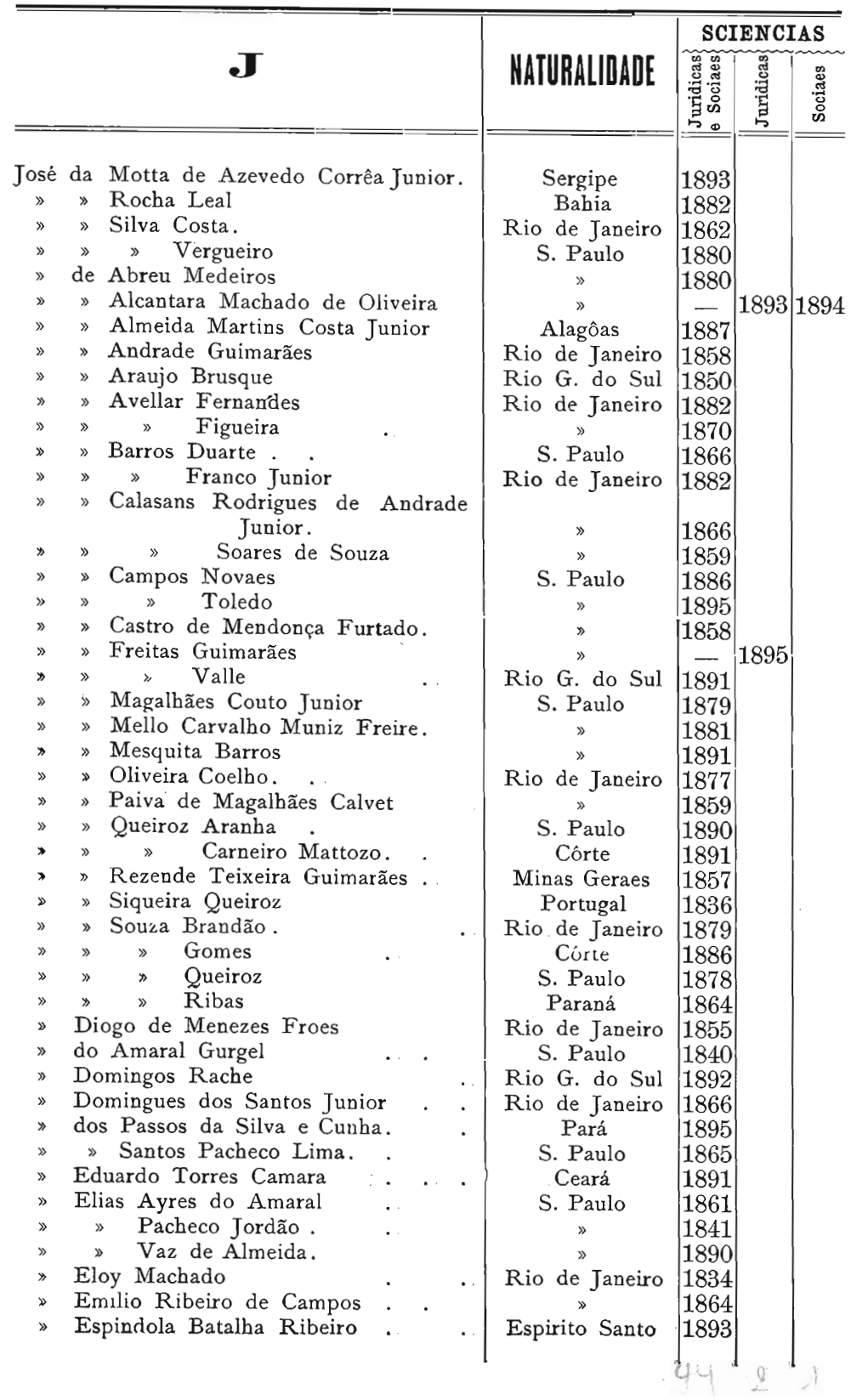




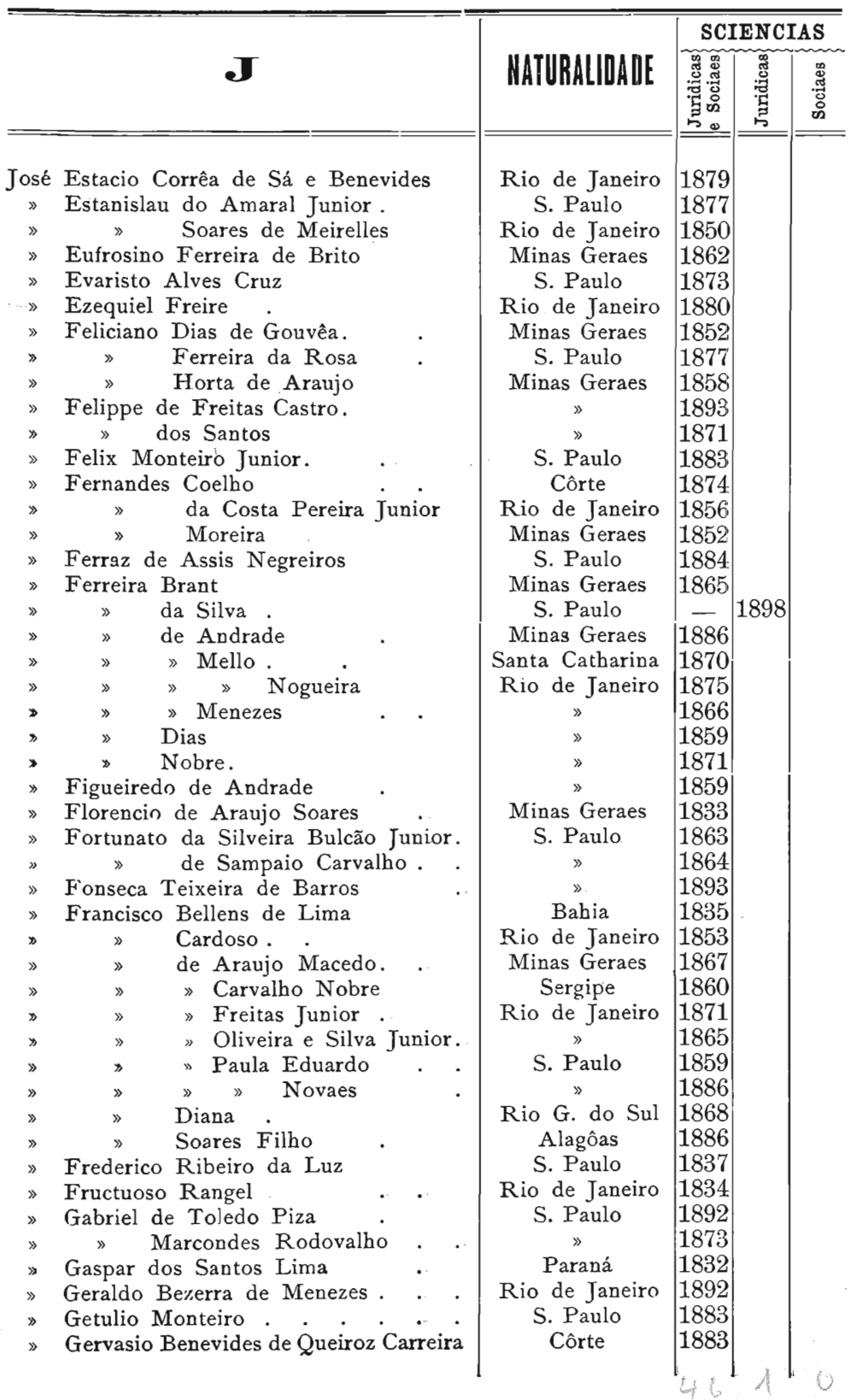




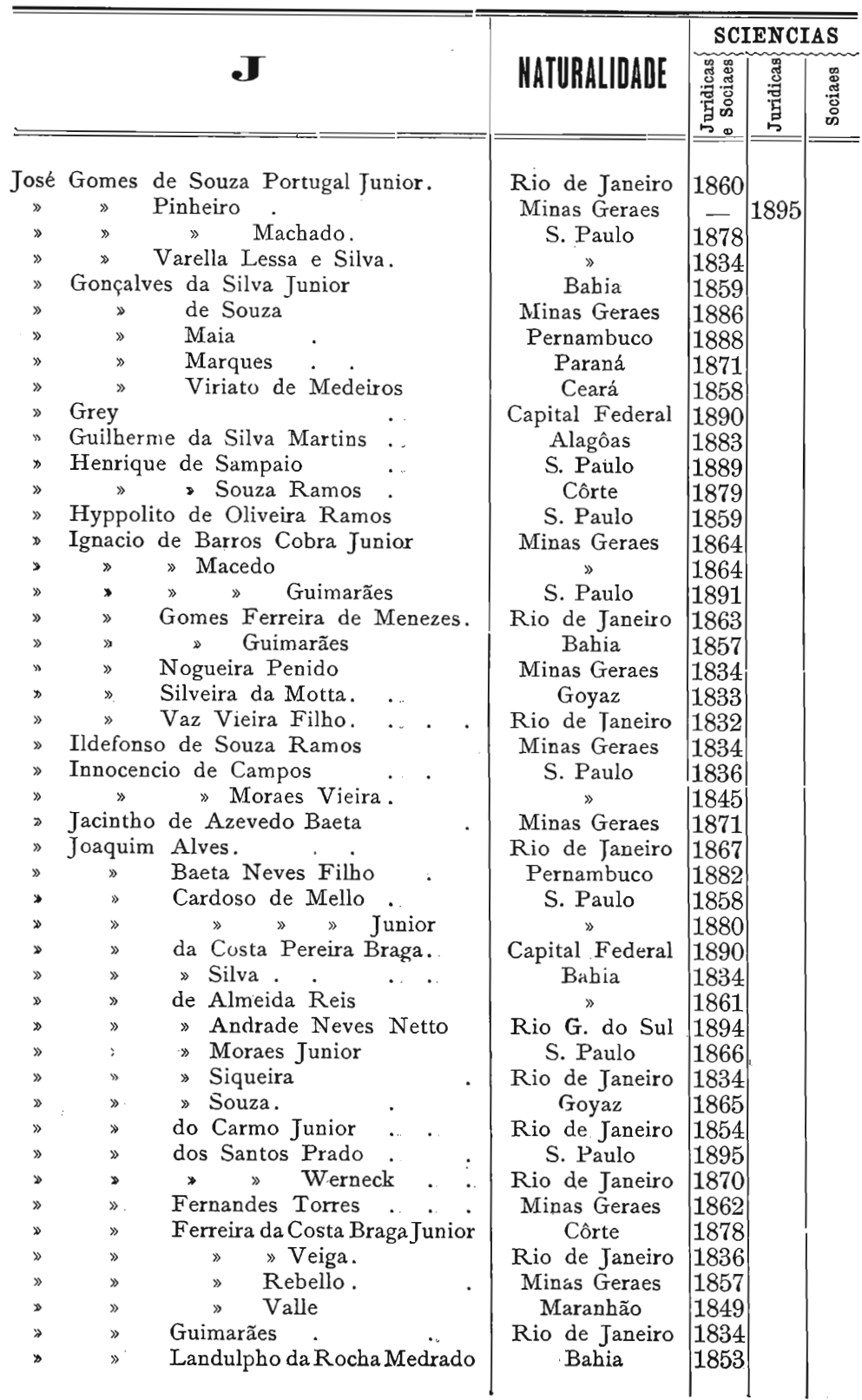




\begin{tabular}{|c|c|c|c|c|c|c|}
\hline \multirow{2}{*}{\multicolumn{2}{|c|}{$\Xi$}} & & \multirow[b]{2}{*}{ HATURALIDADE } & \multicolumn{3}{|c|}{ SCIENCIAS } \\
\hline & & & & 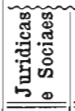 & 营 & 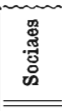 \\
\hline José & Joaquim Machado & & Rio de Janeiro & 1835 & & \\
\hline$»$ & 》 Monteiro de Andrade & & Minas Geraes & 1891 & & \\
\hline$\gg$ & Pessanha Povôa & & Rio de Janeiro & 1864 & & \\
\hline & Jorge da Silva & & Minas Geraes & 1833 & & \\
\hline$»$ & $\gg » \gg$ Junior & & Rio de Janeiro & 1859 & & \\
\hline$\gg$ & Julio de Freitas Coutinho. & & Portugal & 1838 & & \\
\hline & Ladislau Terra. & & Montevidéo & 1864 & & \\
\hline$»$ & Leite de Arruda & & S. Paulo & - & 1895 & \\
\hline$»$ & $\gg \quad$ Souza & & $»$ & - & 1894 & \\
\hline$"$ & Pinheiro & & $»$ & 1890 & & \\
\hline$\rightarrow$ & Leopoldo de Bulhões Jardim & & Goyaz & 1880 & & \\
\hline$»$ & » Pereira Lima & & Pará & 1891 & & \\
\hline$\gg$ & Lobo Leite Pereira : & & Rio de Janeiro & 1890 & & \\
\hline » & Lourenço de Sá Ribas. & & S. Paulo & 1849 & & \\
\hline$\gg$ & Luciano da Silva Barbosa. & & , & $1854^{\prime}$ & & \\
\hline$\gg$ & Luiz Alvares da Silva. & & Minas Geraes & 1884 & & \\
\hline$\gg$ & $»$ de Almeida Nogueira & & S. Paulo & 1873 & & \\
\hline$\gg$ & $» \quad »$ Andrade Junior & & Rio de Janeiro & 1859 & & \\
\hline$\gg$ & » dos Santos Teixeira Junior & & 》 & 1860 & & \\
\hline$\gg$ & » Monteiro de Souza & & » & 1861 & & \\
\hline$\gg$ & Lustosa da Cunha Paranaguá. & & Côrte & 1877 & & \\
\hline$>$ & Machado Coelho de Castro & & Rio de Janeiro & 1850 & & \\
\hline$\gg$ & $» \quad$ Pinheiro Lima & & Paraná & 1871 & & \\
\hline$\gg$ & Manoel da Costa Bastos & & Rio de Janeiro & $\mid 1848$ & & \\
\hline$\gg$ & $» \quad »$ Fonseca Leite Junior. & & S. Paulo & 1881 & & \\
\hline$»$ & de Almeida Pereira & & Rio de Janeiro & 1881 & & \\
\hline 》 & » Azevedo Marques. & & S. Paulo & 1886 & & \\
\hline$\gg$ & $»$ Arruda Alvim. & & 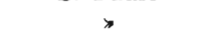 & 1870 & & \\
\hline$\gg$ & » Barros Fonseca & & $\gg$ & & 1895 & 1896 \\
\hline \$ & » Mesquita Junior & & $》$ & 1859 & & \\
\hline$»$ & Freire Junior & & Rio de Janeiro & 1868 & & \\
\hline$\gg$ & Lobo : & & S. Paulo & 1886 & & \\
\hline$»$ & Machado de Araujo Filho & & Sergipe & 1896 & & \\
\hline$\gg$ & Pereira Cabral & & Minas Geraes & 1861 & & \\
\hline$\gg$ & \} \quad \text { Portugal } \quad . \quad \text { . . } $&{ } &{\text { Rio de Janeiro }} &{1857} &{ } &{ } \\
{\hline \gg} &{\text { Marcellino de Araujo Ledo Vega }} &{ } &{\text { 》 }} &{1863} &{ } &{ } \\
{\hline »} &{\text { Marciano Gomes Baptista }\left(\mathrm{P} .^{\left.{ }^{\circ}\right)}{ }^{\circ}\right.} &{ } &{\text { Minas Geraes }} &{1834} &{ } &{ } \\
{\hline \gg} &{\text { Marcondes de Andrade Figueira }} &{ } &{\text { Rio de Janeiro }} &{1882} &{ } &{ } \\
{\hline \gg} &{\text { Maria Bourroul. . . }} &{ } &{\text { S. Paulo }} &{1884} &{ } &{ } \\
{\hline \gg} &{\text { » Corrêa de Sá e Benevides. }} &{ } &{\text { Rio de Janeiro }} &{1854} &{ } &{ } \\
{\hline »} &{\text { > da Camara Leal }} &{ } &{\gg} &{1856} &{ } &{ } \\
{\hline \gg} &{\text { 》 de Andrade. . . . . }} &{ } &{\text { S. Paulo }} &{1851} &{ } &{ } \\
{\hline \gg} &{» \quad » \text { Azevedo Velho Junior: }} &{ } &{\text { Rio de Janeiro }} &{1859} &{ } &{ } \\
{\hline \gg} &{\text { » Campos Cordeiro }} &{ } &{\text { Minas Geraes }} &{1879} &{ } &{ } \\
{\hline »} &{\text { » Moura Leite. }} &{ } &{\text { 》 }} &{1862} &{ } &{ } \\
{\hline \text { » }} &{\triangleright \gg \quad \text { Junior }} &{ } &{»} &{-} &{1895} &{ } \\
{\hline \gg} &{» \text { Oliveira . }} &{ } &{\text { 》 }} &{1894} &{ } &{ } \\
{\hline \text { ( }} &{!} &{ } &{ } &{ } &{ } &{ } \\
$\hline
\end{tabular}




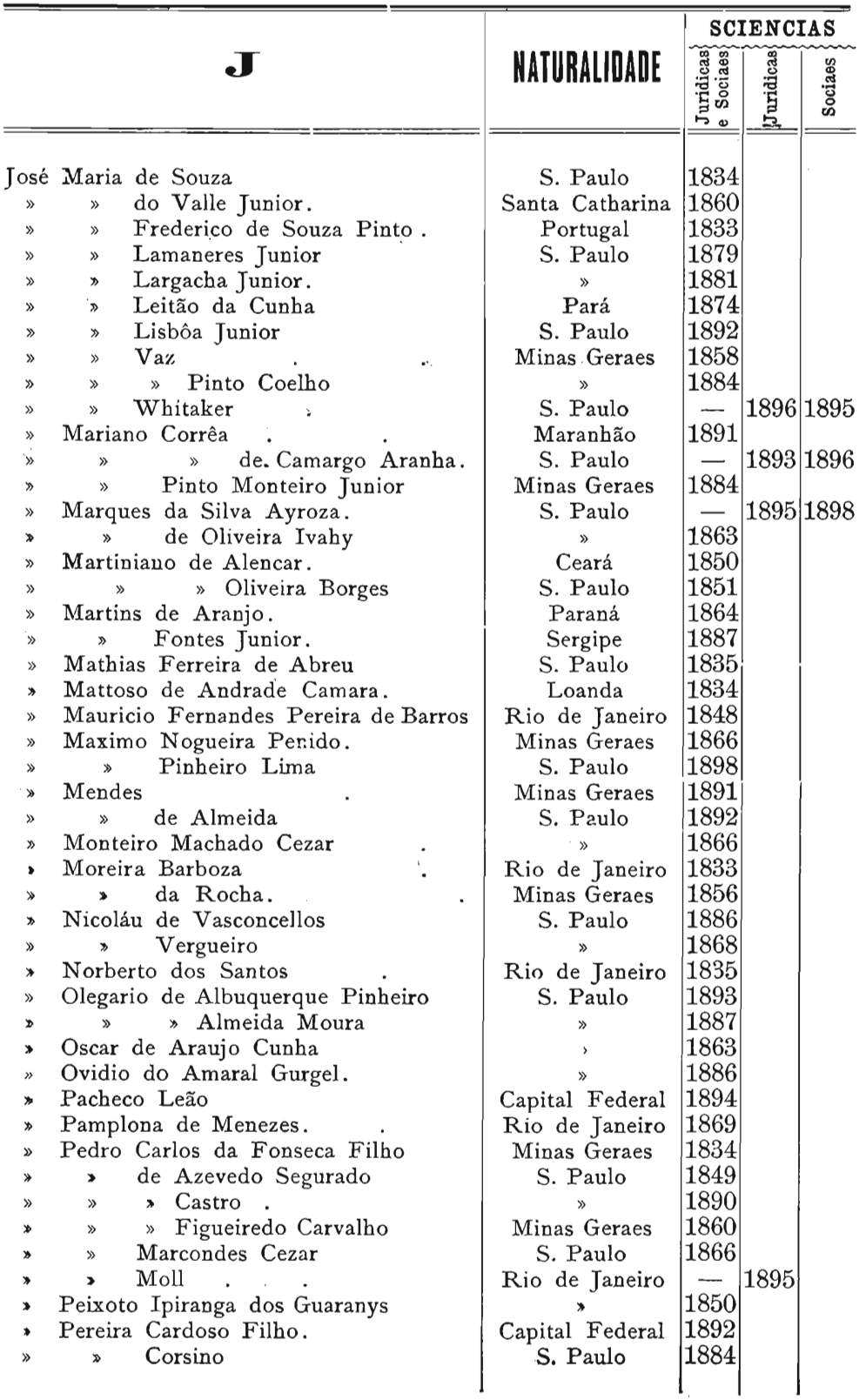




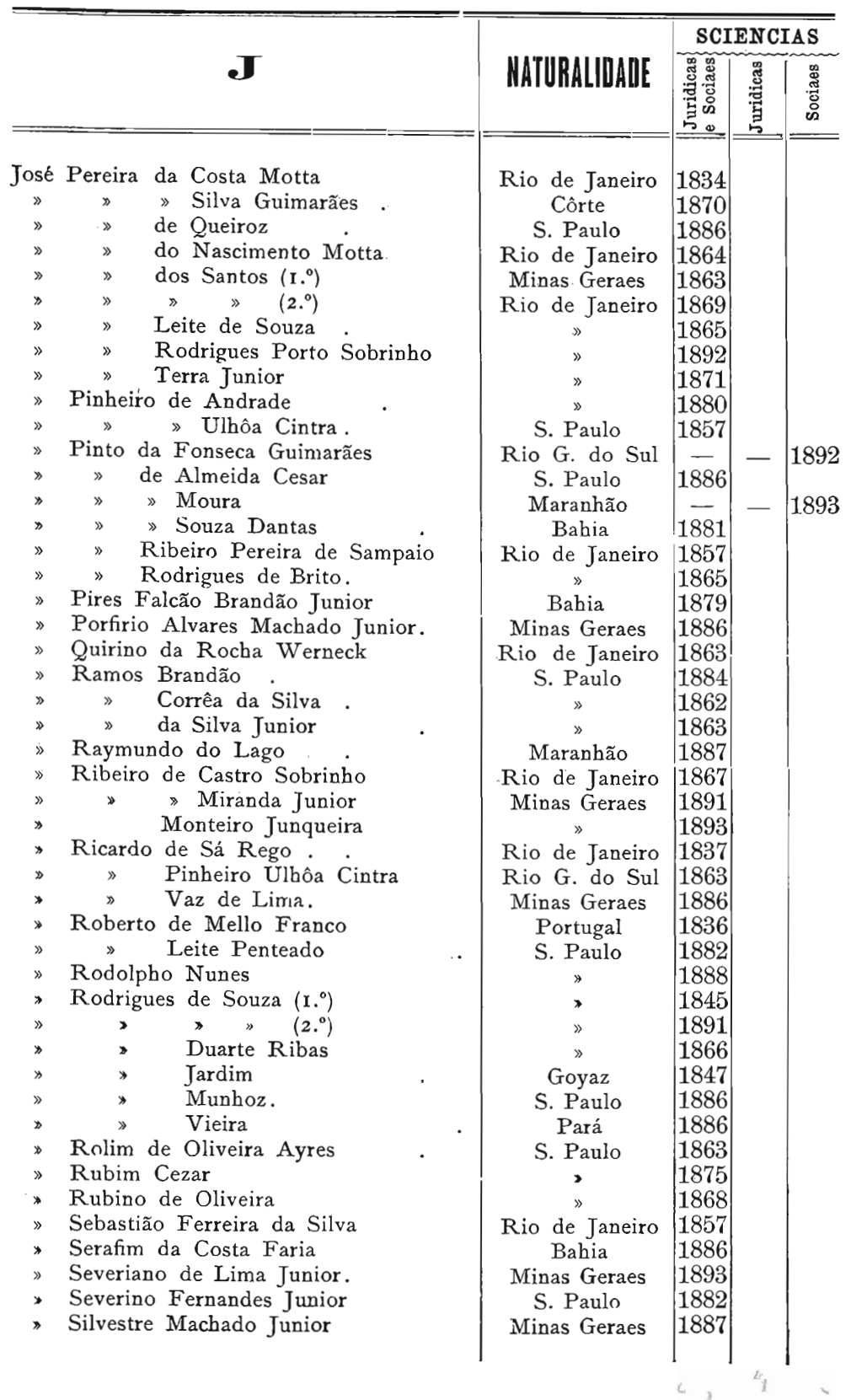




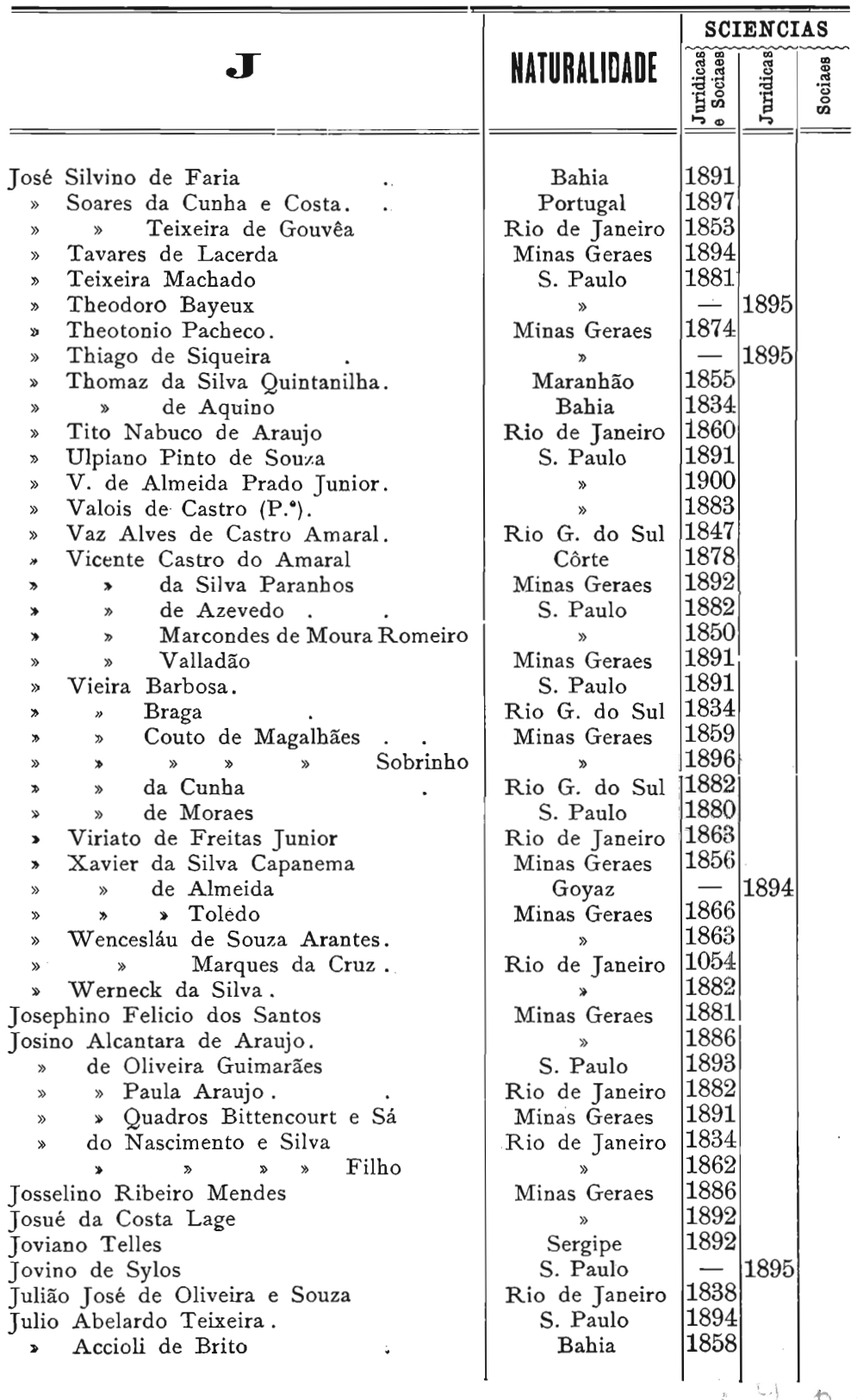




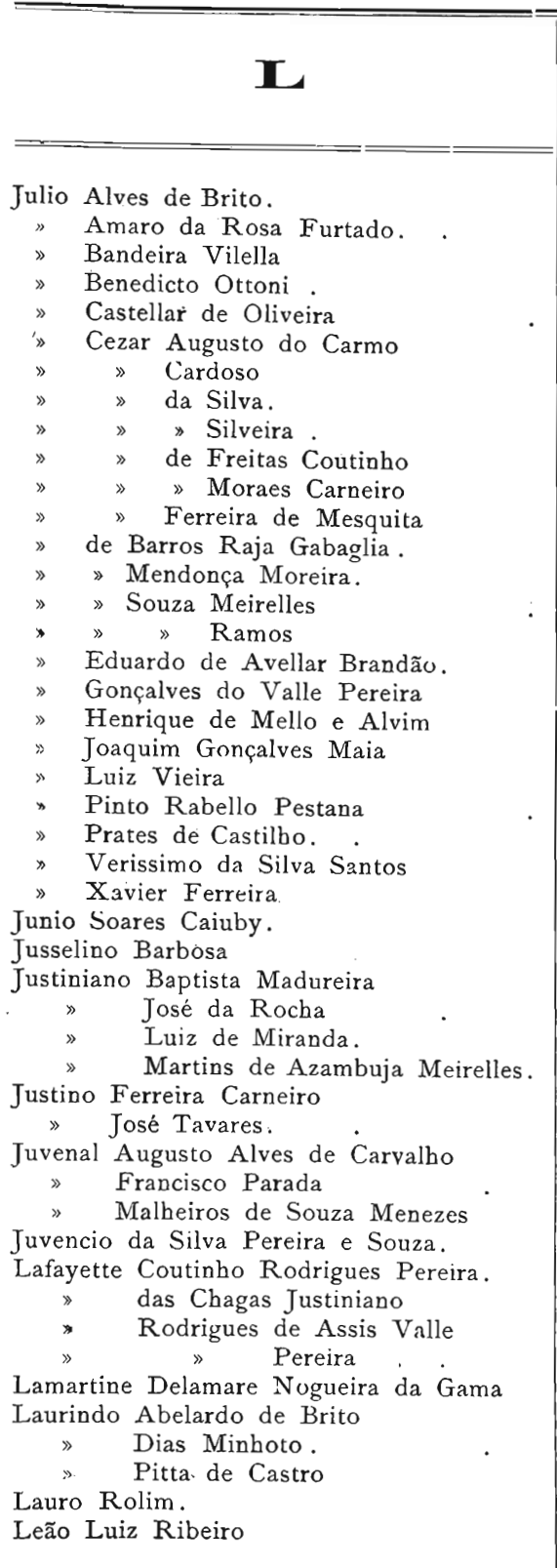

\section{NATURALIDADE}

SCIENCIAS

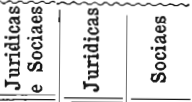

1874

S. Paulo

1887

Ceará

1893

Côrte

1878

S. Paulo

1896

Rio de Janeiro 1863

Pernambuco 1893

S. Paulo

- 1895

Santa Catharina Côrte

1869

Rin de Janeiro $\mathbf{1 8 7 4}$

S. Paulo 1883

Rio de Janeiro $\mathbf{1 8 8 6}$

Rio G. do Sul 1877

Minas Geraes 1891

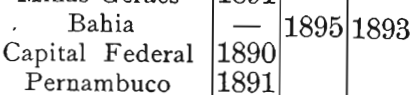

Rio de Janeiro 1854

S. Paulo 1884

Rio de Janeiro 1863

S. Paulo 1866

Rio G. do Sul 1881

Rio de Janeiro 1883

S. Paulo

1856

Minas Geraes

\begin{tabular}{c|c} 
Bahia & 1846
\end{tabular}

1890

Rio de Janeiro 1833

Minas Geraes 1835

S. Paulo

Minas Geraes

Rio de Janeiro

S. Paulo

1876

》

1886

Rio de Janeiro

Minas Geraes

1868

$»$

1893

$\gg$

'

Montevidéo

S. Paulo

1851

Rio de Janeiro

S. Paulo

1898

Rio G. do Sul 


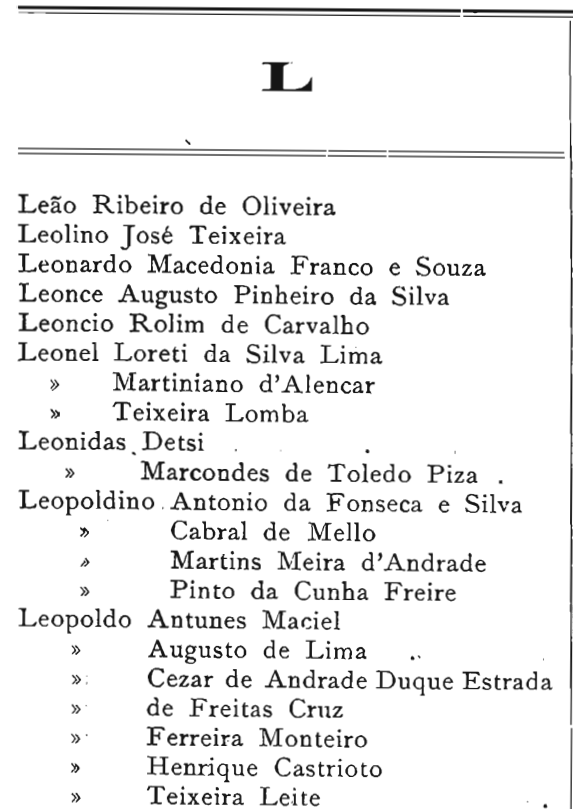

Leovegildo de Mendonça Uchôa

Levindo Ferreira Lopes.

Liberato da Costa Fontes

Lincoln Guimarães

Lindolf de Vereza Soares » Ernesto Ferreira França

Lindolpho d'Almeida Campos

Lopo Diniz Cordeiro

Loreto Ribeiro de Abreu

Lourenço de Mello Franco » Justiniano dia Silva

\Valente de Figueiredo

Lucas Jorge de Siqueira Franco.

Luciano de Souza Lima Netto.

» Esteves dos Santos Junior

» Rangel d'Azevedo.

Lucio da Cunha Pavolid e Menezes (Dr.). de Toledo Malta

. Drummond Furtado de Mendonça

» Pereira Peixoto

Ludgero Antonio Coelho

Luiz Adolpho Thiers Velloso

* Albino Barboza de Oliveira

»Alves da Silva Carvalho

" de Souza

» $\gg$ Leite de Oliveira Bello

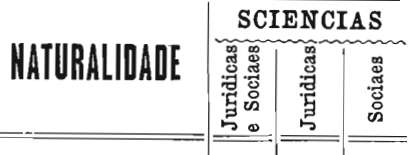 \\ Minas Geraes - 1899 \\ Bahia - 1895 \\ Rio G. do Sul 1891 \\ S. Paulo 1878 \\ " 1897}

Rio de Janeiro 1884

" 1853

Minas Geraes 1866

Espirito Santo 1892

Rio de Janeiro 1866

Alagôas

Rio de Janeiro 1887

S. Paulo 1883

» 1893

Rio G. do Sul 1870

Minas Geraes 1891

Rio de Janeiro 1868

Rio G. do Sul 1891

Minas Geraes 1887

Rio de Janeiro 1858

, 1881

Alagôas 1882

Rio de Janeiro 1866

Sergipe 1891

Rio de Janeiro 1897

S. Paulo 1863

» 1856

Minas Geraes - 1895

Rio de Janeiro 1856

Minas Geraes 1890

S. Paulo 1835

Rio de Janeiro 1837

$\begin{array}{ll}\text { Piauby } & 1862\end{array}$

S. Paulo 1897

Minas Geracs 1887

S. Paulo 1896

Minas Geraes 1863

Capital Federal 1895

S. Paulo 1866

Rio de Janeiro 1877

S. Paulo 1895

Minas Geraes 1884

Bahia 1894

Côrte 1878

Rio de Janeiro 1866

Côrie 1869

Rio G. do Sul 1841 


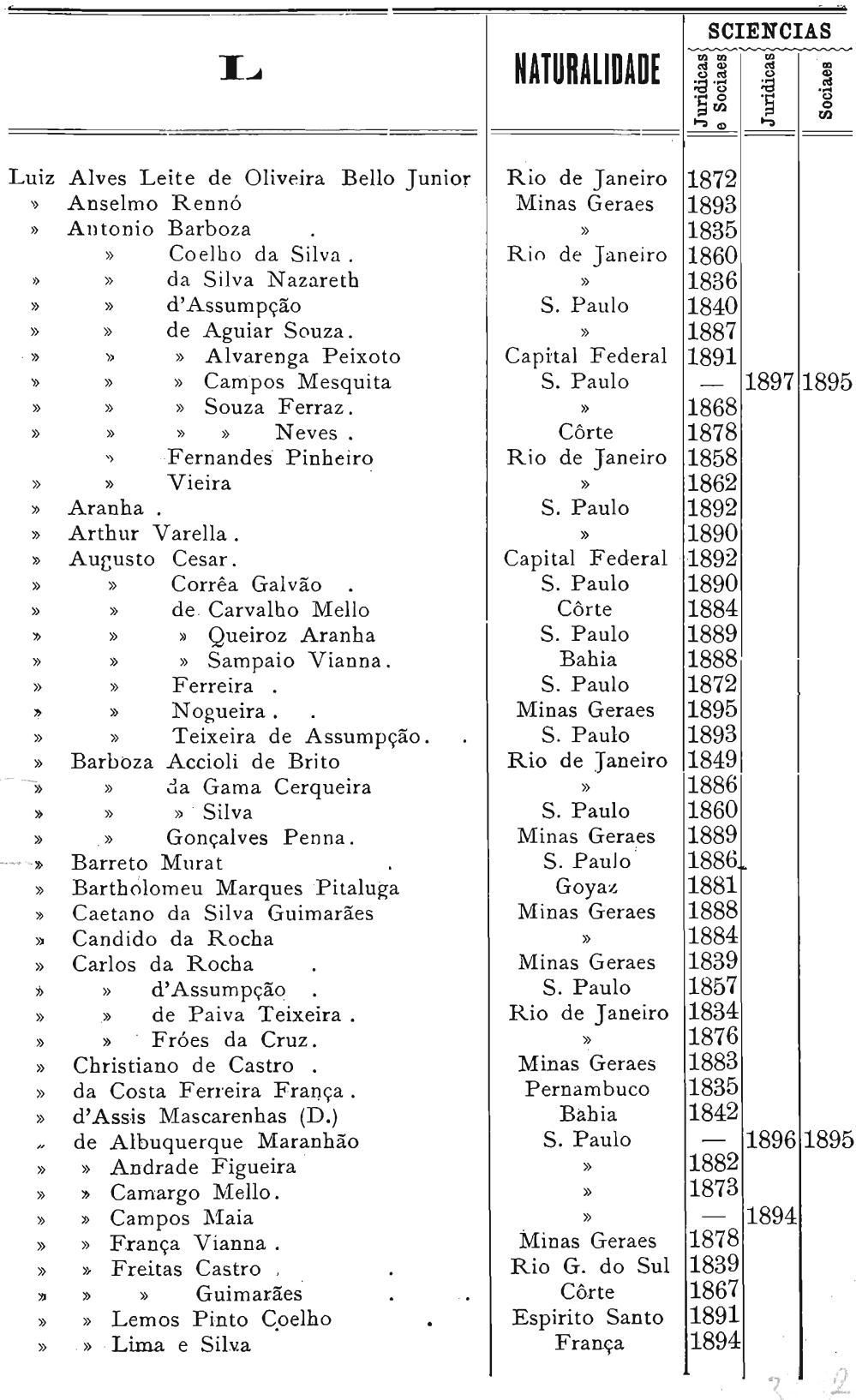




\begin{tabular}{|c|c|c|c|c|c|}
\hline \multirow{2}{*}{\multicolumn{2}{|c|}{ II }} & \multirow[b]{2}{*}{ NATURALIDADE } & \multicolumn{3}{|c|}{ SCIENCIAS } \\
\hline & & & 范 & 菢 & 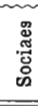 \\
\hline Luiz & de Medeiros & Santa Catharina & 1855 & & \\
\hline & » Oliveira Lins e Vasconcellos & Alagôas & 1867 & & \\
\hline & $\triangleright$ Rezende e Souza & S. Paulo & 1894 & & \\
\hline$»$ & »Siqueira da Silva Lima & Espirito Santo & 1868 & & \\
\hline & $»$ Souza Dias & Rio de Janeiro & 1890 & & \\
\hline$\gg$ & $» \quad$ Leite Junior. & S. Paulo & 1895 & & \\
\hline 》 & '» Monteiro de Barros & Rio de Janeiro & 1870 & & \\
\hline & » Toledo Piza e Almeida & S. Paulo & 1883 & & \\
\hline & Dias Novaes & 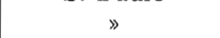 & 1856 & & \\
\hline$»$ & Edmundo Cases & $»$ & 1879 & & \\
\hline$»$ & Ernesto Xavier & $»$ & 1863 & & \\
\hline$»$ & Eugenio Horta Barbo\%a & Minas Geraes & 1863 & & \\
\hline$\gg$ & Fernandes Ferreira & Maranhão & 1837 & & \\
\hline$\gg$ & Ferreira da Silva Maia & Rio de Janeiro & 1837 & & \\
\hline$»$ & » Garcia. & S. Paulo & 1881 & & \\
\hline$»$ & Gomes & Rio de Janeiro & 1835 & & \\
\hline$»$ & Felippe Baeta Neves & Minas Geraes & 1886 & & \\
\hline$»$ & $\begin{array}{c}\text { Fortunato de Brito Abreu Souza Me- } \\
\text { nezes. }\end{array}$ & Rio de Janeiro & 1832 & & \\
\hline " & $\begin{array}{l}\text { Fortunato de Brito Abreu Souza Me- } \\
\text { nezes Filho }\end{array}$ & » & 1863 & & \\
\hline » & Fortunato de Souza Carvalho. & S. Paulo & 1893 & & \\
\hline » & Francisco da Camara Leal & Rio de Janeiro & 1845 & & \\
\hline$\gg$ & $» \quad$ Fontoura Lima. & $\gg$ & 1864 & & \\
\hline$»$ & Frederico Rangel de Freitas & S. Paulo & 1889 & & \\
\hline$»$ & Gastão d'Escragnolle Doria & Capital Federal & 1890 & & \\
\hline$»$ & Gomes Martins. & Minas Geraes & 1882 & & \\
\hline$»$ & » Ribeiro. & $»$ & 1857 & & \\
\hline » & Gonzaga da Silva Leme & S. Paulo & 1876 & & \\
\hline$»$ & $»$ de Oliveira Costa & $»$ & 1870 & & \\
\hline$»$ & Jayme & Goyaz & 1882 & & \\
\hline$»$ & Pereira da Fonseca & Minas Geraes & 1868 & & \\
\hline$»$ & Guedes de Moraes Sarmento Junior & Capital Federal & 1891 & & \\
\hline$»$ & Ignacio Nascentes d'Azambuja & Rio de Janeiro & 1836 & & \\
\hline$»$ & Joaquim de Magalhães Castro Junior. & Babia & 1863 & & \\
\hline$》$ & » Duque Estrada Teixeira. & Rio de Janeiro & 1836 & & \\
\hline$»$ & José da Costa e Souza & .. & 1836 & & \\
\hline$»$ & » de Carvalho Mello e Mattos & $»$ & 1860 & & \\
\hline$"$ & „ Mello e Oliveira & S. Paulo & 1862 & & \\
\hline$»$ & $»$ Ferreira Braga . : & Rio de Janeiro & 1866 & & \\
\hline$»$ & $» \quad »$ de Araujo & Espirito Santo & 1851 & & \\
\hline$»$ & Ladisláu de Toledo Dantas & S. Paulo & 1855 & & \\
\hline$»$ & Lopes Baptista dos Anjos Junior & Bahia & 1877 & & \\
\hline » & $»$ Domingues . . & Rio de Janeiro & - & 1895 & \\
\hline$»$ & Mattozo Duque Estrada Camara & $»$ & 1864 & & \\
\hline & Mello Guimarães & Rio G. do Sul & 1897 & & \\
\hline & Nogueira Martins & S. Paulo & - & 1894 & \\
\hline
\end{tabular}




\begin{tabular}{|c|c|c|c|c|c|c|}
\hline & & & & SCI & IENCI & IAS \\
\hline & & & NATHRALIDADE & 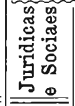 & 总 & 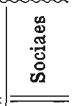 \\
\hline Luiz 1 & Nunes Ferreira Filho & & Rio de Janeiro & 1893 & & \\
\hline & Paulino Percira Pinto. & & Rio G. do Sul & 1876 & & \\
\hline$\gg \quad \mathrm{P}$ & Pedreira do Couto Ferraz. & & Rio de Janeiro & 1838 & & \\
\hline$\gg \mathrm{F}$ & Pereira de Castro Filho & & $»$ & 1864 & & \\
\hline$»$ & $» \quad$ Ferreira de Faro & & Côrte & 1884 & & \\
\hline$\nu$ & Pinto de Alencar Cintra & . & S. Paulo & 1894 & & \\
\hline$»$ & » $\gg$ Miranda Montenegro & . & Rio de Janeiro & 1853 & & \\
\hline$»$ & $»$ Serva & & S. Paulo & 1900 & & \\
\hline$»$ & Porfirio da Rocha. & & Rio de Janeiro & 1864 & & \\
\hline 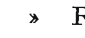 & Ramos da Silva Barbas & : & $»$ & 1862 & & \\
\hline » & $» \quad$ Figueira & & $\gg$ & 1865 & & \\
\hline$»$ & Rodrigues de Lorena Ferreira & & Côrte & 1880 & & \\
\hline$»$ & $» \quad$ Ferreira & & Rio de Janeiro & 1834 & & \\
\hline$»$ & , Filho & & $\gg$ & 1867 & & \\
\hline$»$ & Pereira. & & Minas Geraes & 1890 & & \\
\hline » $\mathrm{F}$ & Romulo Peres de Moreno & & Buenos Ayres & 1859 & & \\
\hline$\gg \mathrm{S}$ & Sanches de Lemos. & & Minas Geraes & 1884 & & \\
\hline$» \mathrm{~S}$ & Serra $\quad$. & & Matto Grosso & 1894 & & \\
\hline$» S$ & Silverio Alves Cruz & & S. Paulo & 1854 & & \\
\hline$\gg \mathrm{S}$ & Sizenando Xavier Serra-dourada & & Goyaz & 1891 & & \\
\hline$» \mathrm{~S}$ & Şoares da Silveira & & S. Paulo & 1897 & & \\
\hline$»$ & » de Gouvêa & . & Minas Geraes & 1843 & & \\
\hline$\gg \mathrm{T}$ & Teixeira de Barros Junior. & & S. Paulo & 1887 & & \\
\hline$" \mathbf{T}$ & Torquato Marques de Oliveira & & Minas Geraes & 1834 & & \\
\hline$\gg \mathrm{T}$ & Tosta da Silva Nunes. & & Parabyba & 1883 & & \\
\hline Luperc & cio da Rocha Lima & & Bahia & 1866 & & \\
\hline Lycurg & go Leite & & Minas Geraes & - & 1898 & 1897 \\
\hline Lysipp & po Antonio do Amaral Garcia & . & Capital Federal & 1893 & & \\
\hline Mamed & de José Gomes da Silva (P.e) & & S. Paulo & 1854 & & \\
\hline Manço: & os Pinto de Andrade & & Minas Geraes & 1893 & & \\
\hline Manoel & el Alvaro de Souza Sá Vianna & & Maranbão & 1883 & & \\
\hline$»$ & Alves Alvim & & S. Paulo & 1832 & & \\
\hline$»$ & $»$ de Araujo & . & Paraná & 1859 & & \\
\hline$》$ & Antonio Braune. & & Rio de Janeiro & 1876 & & \\
\hline$»$ & $» \quad$ dos Passos & & $»$ & 1860 & & \\
\hline$\gg$ & Duarte de Azevedo & & $»$ & 1856 & & \\
\hline$»$ & Dutra Rodrigues & & Côrte & 1878 & & \\
\hline 》 & Pereira Lima & & S. Paulo & 1891 & & \\
\hline$»$ & Augusto de Almeida & & Bahia & 1842 & & \\
\hline$》$ & » Alvarenga. & & S. Paulo & 1881 & & \\
\hline$»$ & Mendonça Brito & & $»$ & 1866 & & \\
\hline$"$ & $»$ Ornellas & & $»$ & 1884 & & \\
\hline & Baptista da Cruz Tamandaré & & Rio de Janeiro & 1858 & & \\
\hline$»$ & Bento Guedes de Carvalho & & S. Paulo & 1839 & & \\
\hline " & Cardoso de Menezes Barretto & & Sergipe & & 1896 & 1895 \\
\hline$»$ & Clementino da Conceição Mo & inte & Alagôas & 1881 & & \\
\hline » & Coelho Barroso. & & Rio de Janeiro & 1872 & & \\
\hline
\end{tabular}




\begin{tabular}{|c|c|c|c|c|c|}
\hline & & & SCI & IENCI & IAS \\
\hline & & NAT|URALIIAADE & 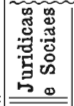 & 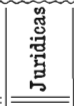 & 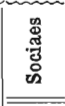 \\
\hline Manoel & Coelho de Almeida. & Rio de Janeiro & 1865 & & \\
\hline$»$ & Corrêa Dias. & S. Paulo & 1879 & & \\
\hline$»$ & d'Almeida Mello Freire & $»$ & 1857 & & \\
\hline$»$ & da Costa Manso & » & 1895 & & \\
\hline$»$ & » Cunba Lopes Vasconcellos. & Bahia & 1864 & & \\
\hline$»$ & "Silva Gouvêa & Minas Geraes & 1859 & & \\
\hline$»$ & $» \quad$ Mafra & Santa Catharina & 1855 & & \\
\hline$»$ & » Silveira Corrêa & S. Paulo & - & 1895 & \\
\hline » & de Araujo da Cunba & Maranbão & 1853 & & \\
\hline$»$ & »Azevedo Castro. & S. Paulo & - & 1896 & 1897 \\
\hline$»$ & »Campos Cartier & Rio G. do Sul & 1883 & & \\
\hline$»$ & "Freitas Paranhos & Bahia & 1884 & & \\
\hline$»$ & » Jesus Valdetaro. & Rio de Janeiro & 1832 & & \\
\hline$»$ & "Magalhães Gomes & Minas Geraes & 1881 & & \\
\hline$»$ & » Meirelles Freire. & S. Paulo & 1834 & & \\
\hline » & ». Moraes Barros & $»$ & 1857 & & \\
\hline$»$ & » Siqueira Cavaicanti. & Pernambuco & 1883 & & \\
\hline$》$ & Dias da Silva & S. Paulo & 1857 & & \\
\hline$»$ & " de Aquino e Castro & Goyaz & 1879 & & \\
\hline$»$ & $» \quad »$ Toledo & S. Paulo & 1832 & & \\
\hline$»$ & $\gg \gg \quad$ Junior. & $\gg$ & 1862 & & \\
\hline$»$ & Domingues de Castro & $》$ & 1862 & & \\
\hline$\gg$ & do Nascimento da Fonseca Galvão & Sergipe & 1858 & & \\
\hline$»$ & $» \quad » \quad$ Silva & Rio de Janeiro & 1862 & & \\
\hline$»$ & Eduviges de Queiroz Vieira & $\gg$ & 1881 & & \\
\hline$»$ & Elisiario de Castro Menezes & Ceará & 1838 & & \\
\hline$»$ & Eloy dos Santos Andrade. & Minas Geraes & - & 1894 & \\
\hline$»$ & Emilio Gomes de Carvalho & Rio de Janeiro & 1882 & & \\
\hline$»$ & Epiphanio de Andrade. & $»$ & 1891 & & \\
\hline$》$ & Estevam do Espirito Santo & Minas Geraes & 1890 & & \\
\hline$»$ & Eufrasio Corrêa. & Paraná & 1862 & & \\
\hline$»$ & Eustachio Martins de Andrade & Minas Guraes & 1870 & & \\
\hline$»$ & Faustino Corrêa Brandão Junior & $》$ & 1895 & & \\
\hline$»$ & Ferraz de Campos Leite & S. Paulo & 1883 & & \\
\hline » & $\gg \gg \gg$ Salles & $»$ & 1863 & & \\
\hline$»$ & Ferreira de Faria Junior & Côrte & 1865 & & \\
\hline$\gg$ & $»$ Mattos. & Rio de Janeiro & 1859 & & \\
\hline$»$ & $» \quad$ Mello & Santa Catharina & 1872 & & \\
\hline$\triangleright$ & Forquim d'Almeida & Minas Geraes & 1865 & & \\
\hline$»$ & $\gg$ de Campos & Rio de Janeiro & 1866 & & \\
\hline$»$ & Francisco Corrêa & Paraná & 1854 & & \\
\hline » & » da Cruz Tamandaré. & S. Paulo & 1882 & & \\
\hline$»$ & »Silva Guerra. & $\gg$ & 1894 & & \\
\hline$»$ & $» \quad$ de Paula Bittencourt & Rio de Janeiro & 1864 & & \\
\hline$»$ & Frederico da Costa Pinto & Minas Geraes & 1839 & & \\
\hline$»$ & Galeão Carvalhal & S. Paulo & 1893 & & \\
\hline$>$ & Gestoira Passøs. & Rio de Janeiro & 1862 & & \\
\hline
\end{tabular}




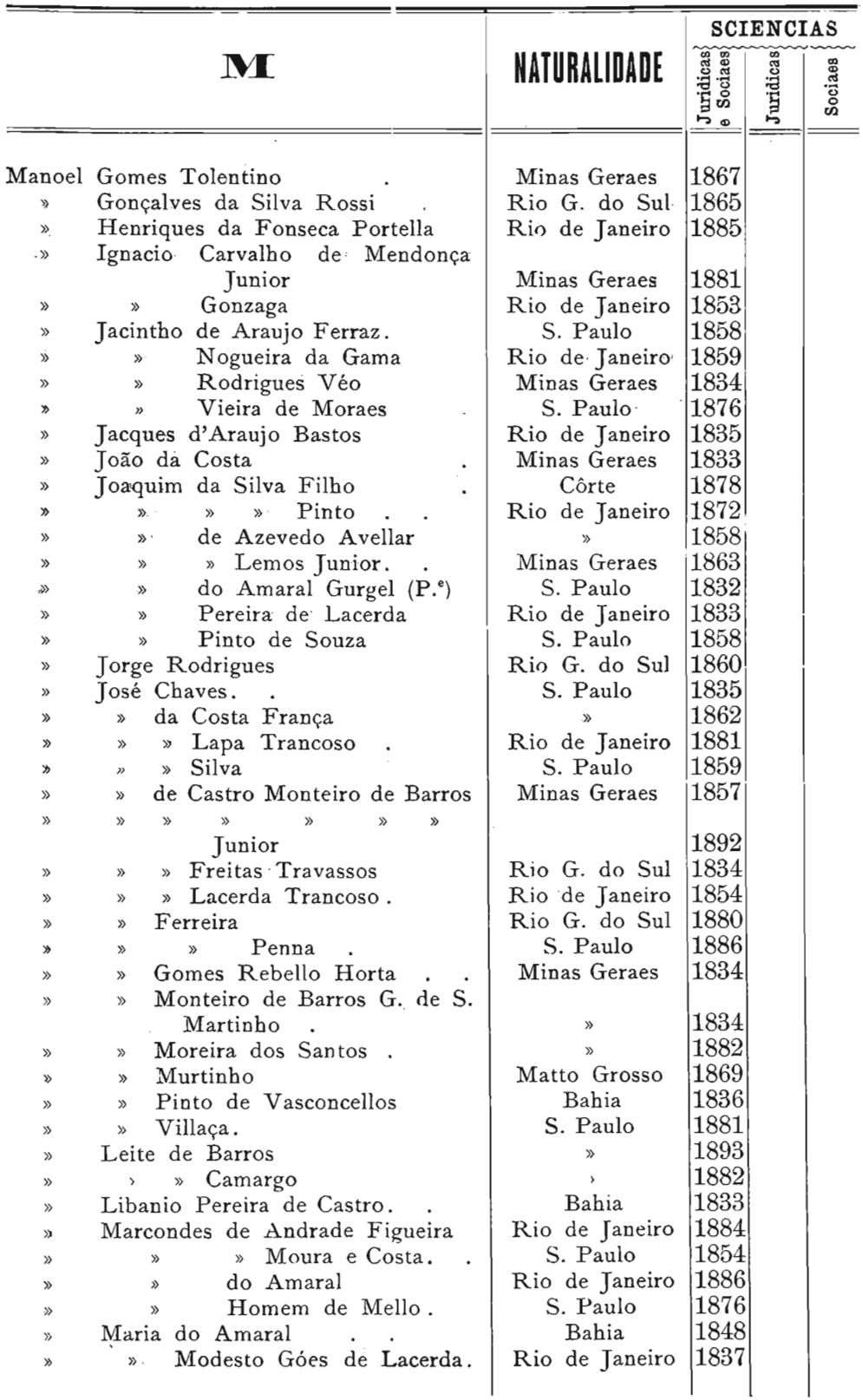




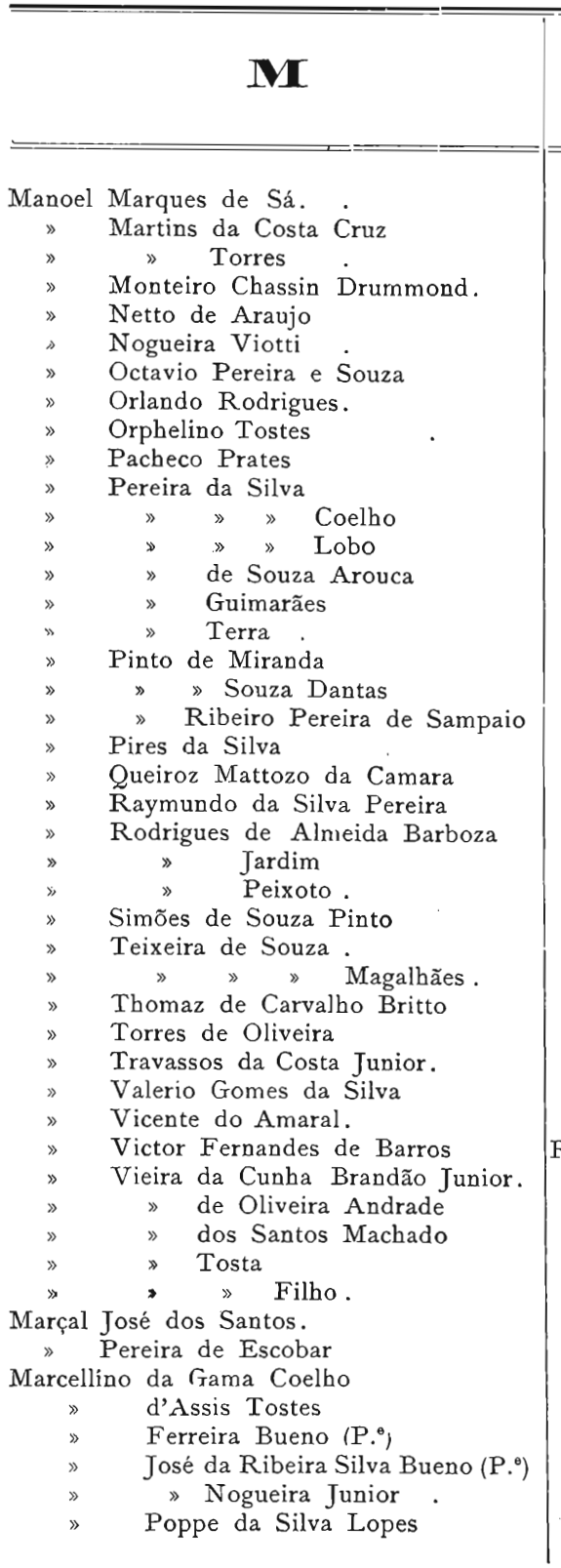

\begin{tabular}{|c|c|c|c|}
\hline \multirow[b]{2}{*}{ NATURALIDADE } & \multicolumn{3}{|c|}{ SCIENCIAS } \\
\hline & 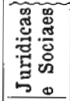 & 营 & 总 \\
\hline Rio de Janeiro & 1856 & & \\
\hline Minas Geraes & 1891 & & \\
\hline Rio de Janeiro & 1864 & & \\
\hline Minas Geraes & 1869 & & \\
\hline S. Paulo & 1877 & & \\
\hline Minas Geraes & 1895 & & \\
\hline Rio de Janeiro & 1891 & & \\
\hline 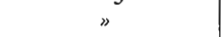 & 1891 & & \\
\hline Rio G. do Sul & 1886 & & \\
\hline$\gg$ & 1886 & & \\
\hline Piauhy & 1842 & & \\
\hline Matto Grosso & 1836 & & \\
\hline Bahia & 1835 & & \\
\hline S. Paulo & 1862 & & \\
\hline$»$ & 1887 & & \\
\hline Rio de Janeiro & 1868 & & \\
\hline , & 1835 & & \\
\hline Bahia & 1872 & & \\
\hline Rio de Janeiro & 1853 & & \\
\hline$»$ & 1866 & & \\
\hline$»$ & 1863 & & \\
\hline Bahia & 1886 & & \\
\hline S. Paulo & 1834 & & \\
\hline Goyaz & 1854 & & \\
\hline Rio de Janeiro & 1864 & & \\
\hline Portugal & 1859 & & \\
\hline Minas Geraes & 1893 & & \\
\hline$»$ & 1862 & & \\
\hline$\gg$ & 一 & 1894 & \\
\hline S. Paulo & 1889 & & \\
\hline Rio de Janeiro & 1833 & & \\
\hline$\gg$ & 1886 & & \\
\hline Rio G. do Sul & 1890 & & \\
\hline Rio G. do Norte & 1877 & & \\
\hline Rio de Janeiro & 1869 & & \\
\hline Minas Geraes & 1891 & & \\
\hline Rंio de Janeiro & 1860 & & \\
\hline Bahia & 1831 & & \\
\hline$»$ & 1860 & & \\
\hline Minas Geraes & 1835 & & \\
\hline Rio G. do Sul & 1878 & & \\
\hline Rio de Janeiro & 1875 & & \\
\hline Minas Geraes & 1862 & & \\
\hline S. Paulo & 1832 & & \\
\hline$»$ & 1833 & & \\
\hline Paraná & 1887 & & \\
\hline Pernambuco & 1891 & & \\
\hline
\end{tabular}




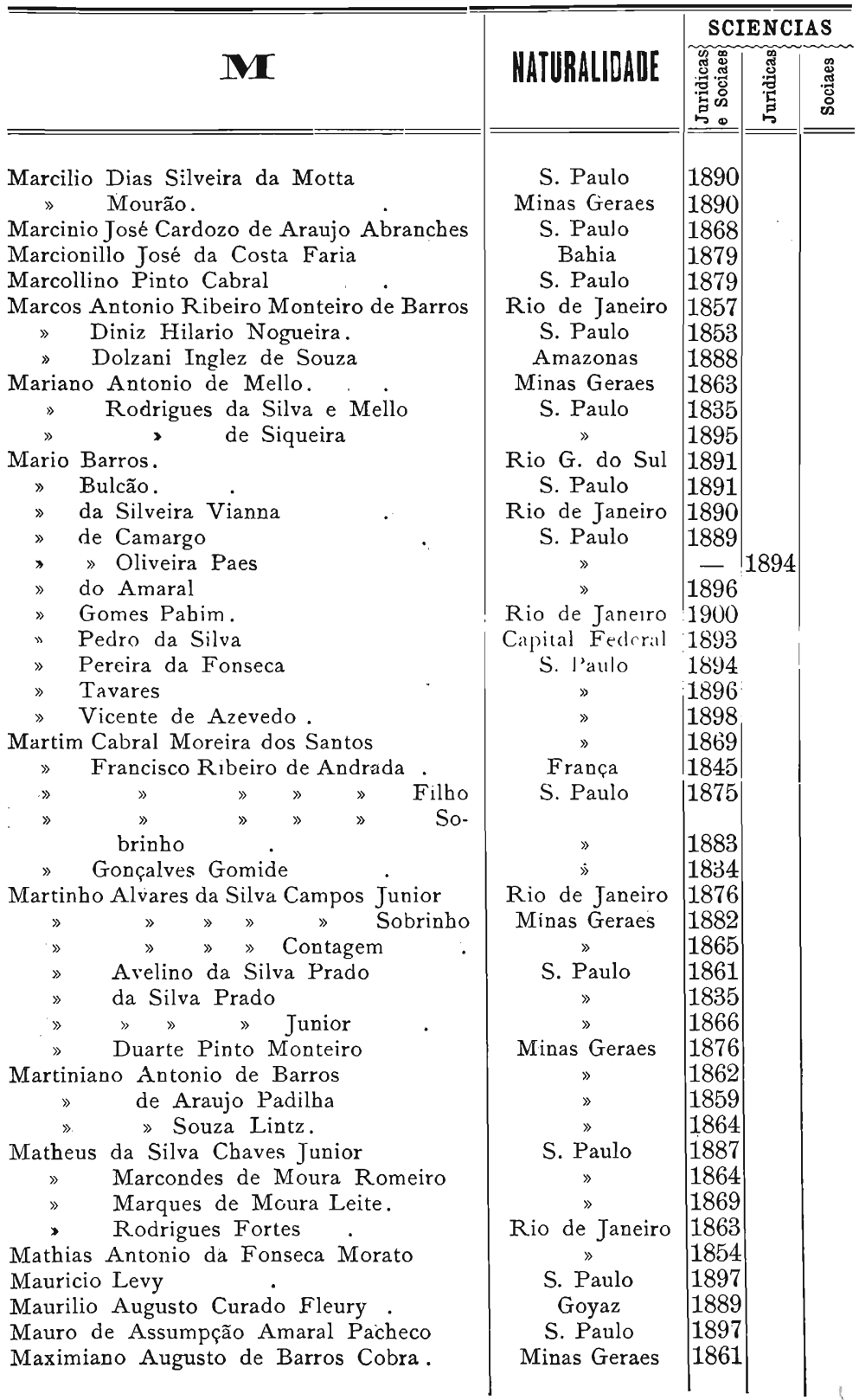




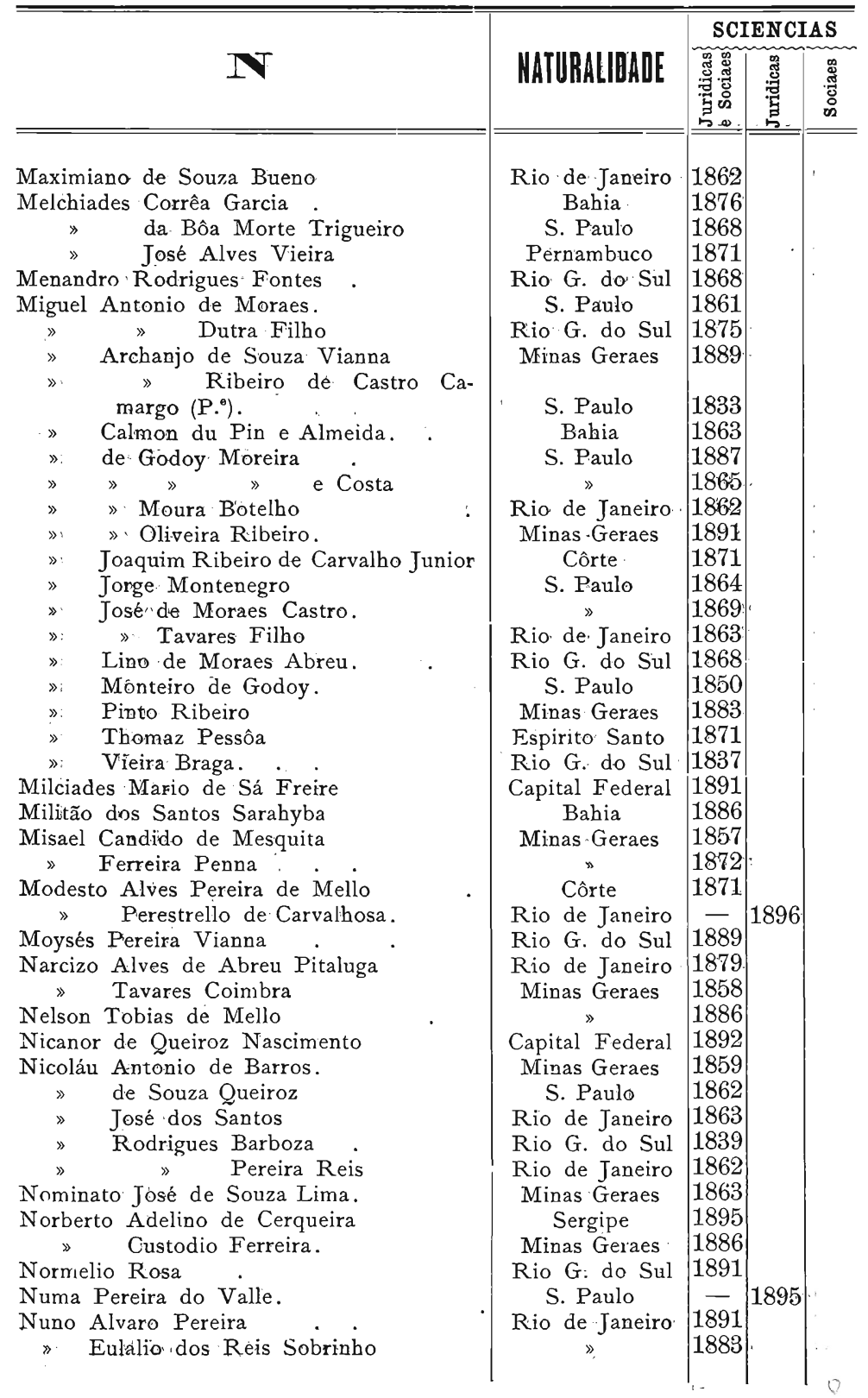




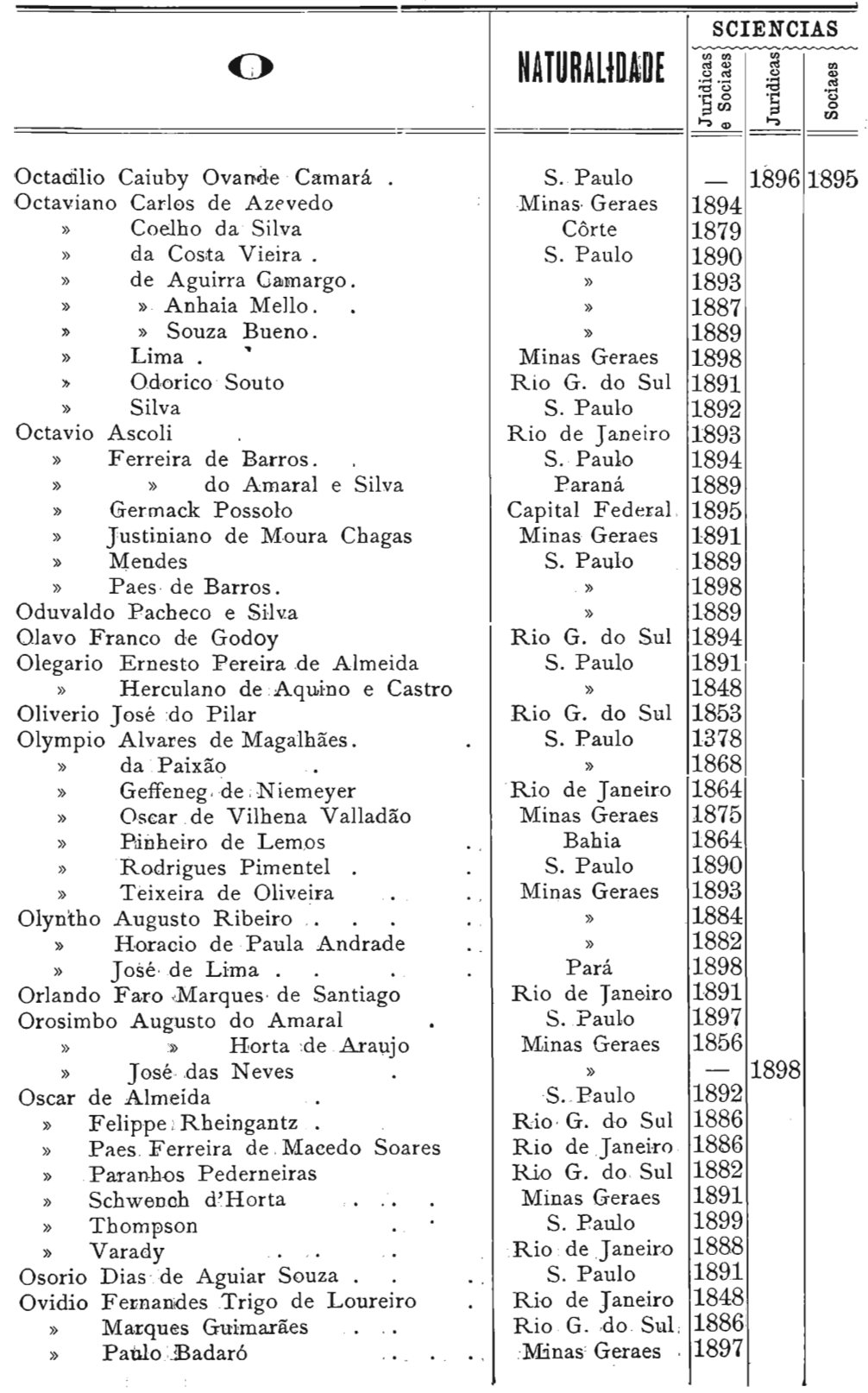




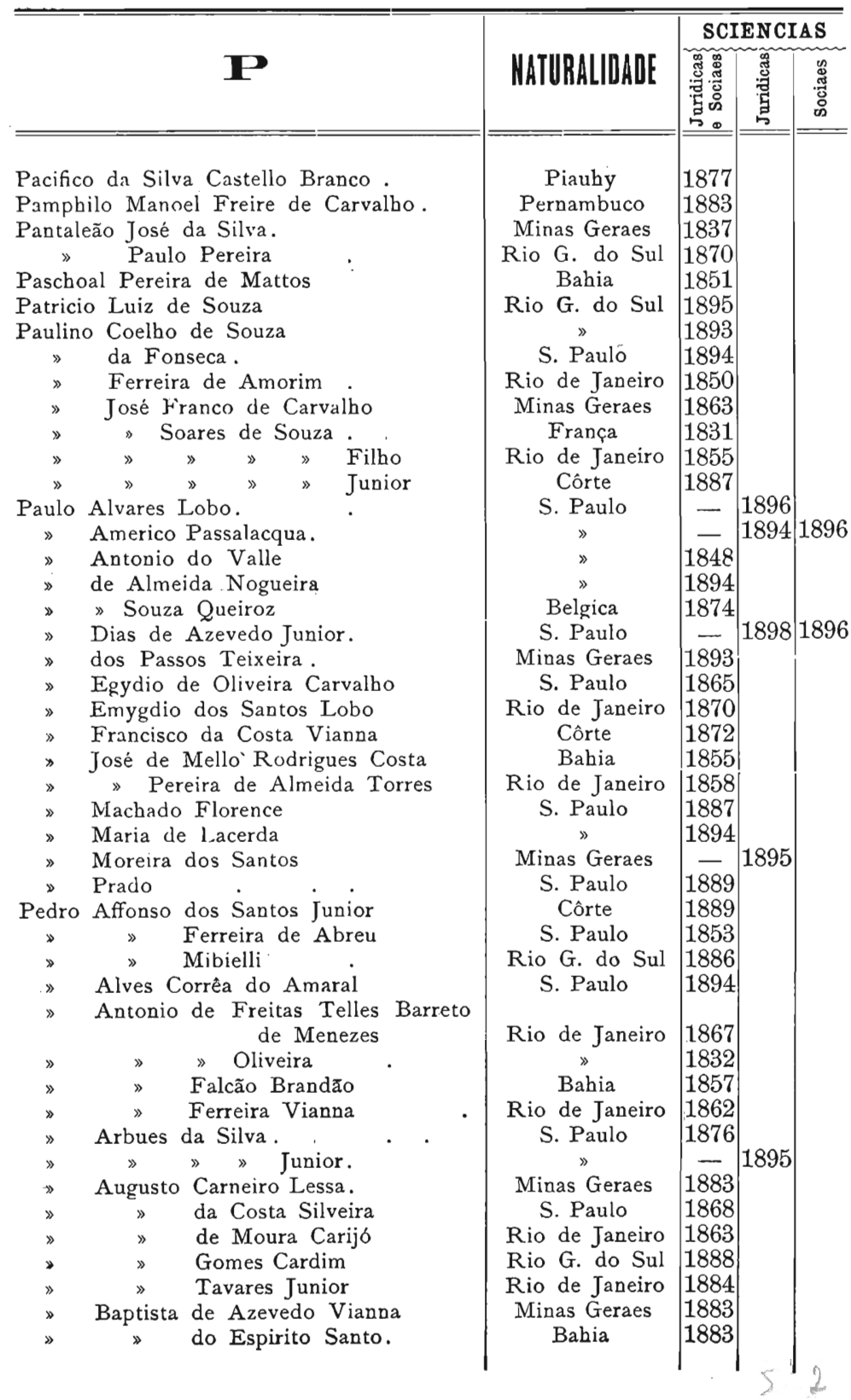




\begin{tabular}{|c|c|c|c|c|c|}
\hline & & & SCI & [ENCI & AS \\
\hline & $1 \mathrm{e}$ & NATIRALIDADE & 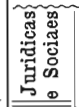 & 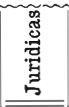 & 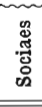 \\
\hline Pedro & Barbosa Nogueira & Côrte & 1884 & & \\
\hline$»$ & Caetano da Costa & Bahia & 1849 & & \\
\hline$»$ & » Sanches de Moura. & Minas Geraes & 1838 & & \\
\hline$»$ & Carvalho Moraes. & Côrte & 1884 & & \\
\hline$»$ & da Costa Fonseça & Minas Geraes & 1834 & & \\
\hline$»$ & » Matta Machado . & $»$ & 1889 & & \\
\hline$»$ & d'Alcantara e Almeida Magalhães & $»$ & 1869 & & \\
\hline$》$ & » Cerqueira Leite & $»$ & 1833 & & \\
\hline » & $\quad$ Leite Ribeiro & Rio de Janeiro & 1884 & & \\
\hline$»$ & Nabuco de Abreu & $\gg$ & 1887 & & \\
\hline 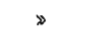 & Sardemberg. & $»$ & 1853 & & \\
\hline$》$ & de Araujo Leite. & Minas Geraes & 1862 & & \\
\hline$»$ & $»$ Arbues Simões Pires & Rio G. do Sul & 1890 & & \\
\hline$»$ & » Castro Canto e Mello. & $»$ & 1891 & & \\
\hline$»$ & »asconcellos Teixeira da Matta & Minas Geraes & 1875 & & \\
\hline$»$ & Dias de Carvalho & Côrte & 1876 & & \\
\hline$》$ & do Monte Ablas. & S. Paulo & - & 1895 & \\
\hline$»$ & Elias Martins Pereira & Minas Geraes & 1859 & & \\
\hline$»$ & Eugenio Cleto & $»$ & 1894 & & \\
\hline$\gg$ & Fernandes Pereira Corrêa & $\gg$ & 1864 & & \\
\hline > & Fernando Paes de Barros & S. Paulo & 1892 & & \\
\hline$»$ & Francelino Guimarães Filbo & Bahia & 1878 & & \\
\hline$»$ & Gomes Pereira de Moraes. & Rio de Janeiro & 1870 & & \\
\hline$»$ & Gonçalves Moacyr & Rio G. do Sul & 1891 & & \\
\hline$》$ & José Netto Teixeira. & Côrte & 1874 & & \\
\hline$\gg$ & Leão de Souza Guaracy. & Minas Geraes & 1893 & & \\
\hline$\gg$ & Luiz de Oliveira Costa & S. Paulo & 1896 & & \\
\hline$\gg$ & $» \gg \quad$ Sayão. & Côrte & 1887 & & \\
\hline$》$ & " Pereira de Souza & Rio de Janeiro & 1860 & & \\
\hline$»$ & Manoel de Toledo & S. Paulo & 1884 & & \\
\hline » & Maria de Oliveira & Rio de Janeiro & 1859 & & \\
\hline$»$ & Mariani Junior . & Bahia & 1878 & & \\
\hline$»$ & Moniz Leão Vellozo & $»$ & 1878 & & \\
\hline » & Nolasco Xavier de Paula & Minas Geraes & 1859 & & \\
\hline$》$ & Paulo Carneiro de Almeida Pereira & Rio de Janeiro & 1884 & & \\
\hline$\gg$ & $»$ de Souza Nogueira & $»$ & 1869 & & \\
\hline$»$ & » do Amaral & $»$ & 1878 & & \\
\hline$\Rightarrow$ & Ramos da Silva. & ע & 1834 & & \\
\hline ע & Rodovalho Marcondes dos Reis & $»$ & 1863 & & \\
\hline 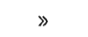 & Rodrigues Fernandes Chaves & Rio G. do Sul & 1832 & & \\
\hline$»$ & $\gg \quad$ Soares de Meirelles. & Côrte & 1869 & & \\
\hline$»$ & Sayão de Bulhões Carvalho. & $»$ & 1883 & & \\
\hline$»$ & Taques d'Almeida Alvim & S. Paulo & 1853 & & \\
\hline ” & Tavares de Almeida. & Pernambuco & 1891 & & \\
\hline$»$ & Teixeira Soares & Rio de Taneiro & 1886 & & \\
\hline$»$ & Vicente de Azevedo. & S. Paulo & 1866 & & \\
\hline$»$ & Xavier Alves Carneiro & Rio de Janeiro & 1854 & & \\
\hline
\end{tabular}




\begin{tabular}{|c|c|c|c|c|}
\hline & & SCI & ENCI & IAS \\
\hline$=1$ & NATURALIDADE & 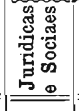 & 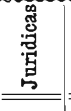 & 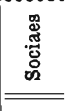 \\
\hline Pedro Wencesláo de Mello e Cunha & Rio de Janeiro. & 1873 & & \\
\hline Pelagio Pereira de Almeida . . & Rio G. do Sul & 1895 & & \\
\hline Pelino Joaquim da Costa Guedes & Pernambuco & 1882 & & \\
\hline Peregrino Vieira da Cunba. & Rio de Janeiro: & 1900 & & \\
\hline Philadelpho de Moraes Lima & S. Paulo & 1884 & & \\
\hline Plinio Alvim. & Rio G. do Sul & 1877 & & \\
\hline $\begin{array}{l}\text { Chaves Barcellos. } \\
» \quad \text { de Castro Casado }\end{array}$ & $\gg$ & $\begin{array}{l}1891 \\
1892\end{array} \mid$ & & \\
\hline » $\gg$ Godoy Moreira e Costa . & S. Paulo & 1893 & & \\
\hline Pompilio de Castro Lima e Almeida & Piaulay & 1891 & & \\
\hline Ponciano Vieira de Araujo Machado & Rio de Janeiro & 1866 & & \\
\hline Porfirio Abdagero Figueira d'Aguiar & S. Paulo & 1871 & & \\
\hline$»$ Ferreira Vellozo & Babia & 1847 & & \\
\hline Possidonio Mancio da Cunha & Rio G. do. Sul & 1884 & & \\
\hline Primitivo de Castro Rodrigues Sette & Ceará & 1886 & & \\
\hline Procopio Corrêa Alves Quintanilha & Rio de Janeiro & 1834 & & \\
\hline$» \quad$ de Toledo Malta & S. Paulo & 1861 & & \\
\hline Prudente José de Moraes. & $»$ & 1863 & & \\
\hline Quintiliano José da Silva & Minas Geraes & 1832 & & \\
\hline Rafael Archanjo Gurgel. & S. Paulo & 1900 & & \\
\hline » Corrêa da Silva Sobrinho & i & 1881 & & \\
\hline$» \quad$ de Sampaio. & ” & 1896 & & \\
\hline Dabney de Avellar Brotero & $»$ & 1856 & & \\
\hline de Abreu : Sampaio Vidal & $»$ & 1891 & & \\
\hline »Aguiar & $»$ & 1883 & & \\
\hline$» \quad » \quad$ Paes de Barros & $»$ & 1858 & & \\
\hline » Almeida Magalhães & Rio: de Janeiro & 1887 & & \\
\hline "Araujo Ribeiro. & Rio G. do Sul & 1833 & & \\
\hline Filho & S. Paulo & 1869 & & \\
\hline Ferraz de Sampaio & $\gg$ & 1883 & & \\
\hline Lopes Branco e Silva & Paraná & 1866 & & \\
\hline Marques Cantinho. & S. Yaulo & 1884 & & \\
\hline Tobias de Aguiar & $»$ & 1857 & & \\
\hline Ralpho Pacheco e Silva & $»$ & - & - & 1895 \\
\hline Ramiro Pereira de Abreu & Goyaz & 187.1 & & \\
\hline Randolpho Augusto de Oliveira Fabrino & Minas Geraes & 1883 & & \\
\hline$» \quad$ Fernandes das Chagas. & $\gg$ & 1892 & & \\
\hline Raul Alvares de Castro. & Rio de Janeiro & 1886 & & \\
\hline Chaves de Camargo & Rio G. do Sul & - & 1895 & 1894 \\
\hline de Regis de Oliveira. & França & - & - & 1895 \\
\hline Fernandes . . & Rio de Janeiro & - & 1898 & 1897 \\
\hline Nogueira Penido & Minas Geraes & 1888 & & \\
\hline Ortiz Monteiro . & S. Paulo & - & 1899 & 1895 \\
\hline » Renato Cardoso de Mello & $»$ & 1896 & & \\
\hline$»$ Soares de Moura. . & Minas Geraes & 1900 & & \\
\hline Raymundo Borges Leal Castello Branco . & Piauby & 1858 & & \\
\hline » da Matta de:Azeveda Corrêa $\left(1 .^{\circ}\right)$ & Maranhão & 1863 & & \\
\hline
\end{tabular}




\begin{tabular}{|c|c|c|c|c|}
\hline \multirow[b]{2}{*}{$\infty$} & \multirow[b]{2}{*}{ NATURALIDADE } & \multicolumn{3}{|c|}{ SCIENCIAS } \\
\hline & & 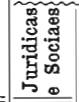 & 营 & 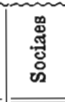 \\
\hline Raymundo da Matta de Azevedo Corrêa (2..$^{\circ}$ ) & Maranhăo & 1883 & & \\
\hline$» \quad \gg$ Silva Cunba Filho & Pará & 1893 & & \\
\hline $\begin{array}{l}\text { Furtado de Albuquerque Ca- } \\
\text { valcanti Filho }\end{array}$ & Paraná & 1892 & & \\
\hline Leonardo Pereira: Brandão. & Minas Geraes & 1891 & & \\
\hline Mártiniano Alves de Souza. & Maranhão & 1863 & & \\
\hline Pennaforte de Toledo Black & S. Paulo & 1889 & & \\
\hline Pereira Smith & Maranhão & 1900 & & \\
\hline Regino Antonio de Carvalho & $\gg$. & 1891 & & \\
\hline Renato Fulton Silveira da Motta & S. Paulo & - & 1898 & 1896 \\
\hline » Gomes Flôres & Capital Federal: & 1890 & & \\
\hline Reynaldo Porchat & S. Paulo & 1891 & & \\
\hline Ricardino Sygmaringa de Moraes Cordeiro. & $»$ & 1890 & & \\
\hline Ricardo Irineo de Soriza & Côrte' & 1879 & & \\
\hline Rivadavia da Cunha Corrêa & Ria G. do Sul & 1887 & & \\
\hline Roberto Jorge Haddoch Lobo. & Côrte & 1886 & & \\
\hline \# Sabiniano de Barros & Minas Geraes & 1869 & & \\
\hline Rodolpho Custodio Ferreira & $»$ & 1886 & & \\
\hline$» \quad$ de Faria Pereira. & $»$ & 1891 & & \\
\hline Ferreira dos Santos. & S. Paulo & 1886 & & \\
\hline Leite Ribeiro & Minas Geraes & 1865 & & \\
\hline Rodrigo Antonio Monteiro de Barros. & S. Paulo & 1854 & & \\
\hline$» \quad$ Augusto da Silva. & » & 1856 & & \\
\hline Bretas de Andrade. & Minas Geraes & 1891 & & \\
\hline Lobato Marcondes Machado. & S. Paulo & 1867 & & \\
\hline Marcondes Romeiro & $»$ & 1886 & & \\
\hline Netto Firmiano de Moraes & Alagôas & 1850 & & \\
\hline Octavio de (Mliveira Menezes. & Bahia & 1861 & & \\
\hline » Langgard Menezes & S. Paulo & 1886 & & \\
\hline Pereira Barreto: & Rio de Janeiro & 1860 & & \\
\hline ». Leite & S. Paulo & 1863 & & \\
\hline $\begin{array}{c}\text { Rogerio O'Connor Lopes de Camargo } \\
\text { Dauntre } .\end{array}$ & $》$ & 1886 & & \\
\hline Pinto Ferraz. & $»$ & 1884 & & \\
\hline Romão Teixeira Leomil Junior: & $»$ & 1882 & & \\
\hline Romualdo de Andrade Baena & Rio de Janeiro & 1874 & & \\
\hline Roque de Souza Dias . & Minas Geraes & 1836 & & \\
\hline Rufino d'Avila Rebouças & Rio de Janeiro & 1846 & & \\
\hline » Furtado de Mendonça & Ceará & 1866 & & \\
\hline » Theotonio Segurado & Minas Geraes & 1843 & & \\
\hline Rufiro Tavares de Almeida Junior. & Parahyba & 1891 & & \\
\hline Ruy Barboza.. . . & Bahia & 1870 & & \\
\hline Sabino Alexandrino Pinheiro Junior & Minas Geraes & 1894 & & \\
\hline » Alves Barroso Junior & $»$ & 1884 & & \\
\hline de Almeida Lustosa & $»$ & 1891 & & \\
\hline Francisco Frougethe & Rio de Janeiro & 1836 & & \\
\hline Gomes da Silva. .. & Babia & 1892 & & \\
\hline
\end{tabular}




\begin{tabular}{|c|c|c|c|c|}
\hline \multirow{2}{*}{$\infty$} & \multirow[b]{2}{*}{ NATURALIIDADE } & \multicolumn{3}{|c|}{ SCIENCIAS } \\
\hline & & 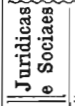 & 萢 & 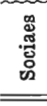 \\
\hline Salathiel Albino de Almeida Cyrino & Minas Geraes & 1886 & & \\
\hline Salustiano de Camargo Penteado & S. Paulo & 1883 & & \\
\hline Salvador Furtado de Mendonça Drummond & Rio de Janeiro & 1869 & & \\
\hline » Leite de Camargo Penteado & S. Paulo & 1877 & & \\
\hline Martins França Junior & Rio G. do Sul & 1862 & & \\
\hline » Meyer de Vasconcellos & S. Paulo & 1884 & & \\
\hline Saturnino Amancio da Silveira. & Minas Geraes & 1866 & & \\
\hline$» \quad$ de Souza e Oliveira. & Rio G. do Sul & 1861 & & \\
\hline Epaminondas de Arruda. & $》$ & 1866 & & \\
\hline Sebastião Eurico Gonçalves de Lacerda & Rio de Janeiro & 1884 & & \\
\hline$\bowtie \quad$ Fleury Curado . & Goyaz & 1886 & & \\
\hline » Fortunato de Oliveira Penteado & S. Paulo & 1884 & & \\
\hline Gomes Barrozo & Rio de Janeiro & 1885 & & \\
\hline José de Carvalho Japejú. & S. Paulo & 1857 & & \\
\hline$\gg$ Pereira & $》$ & 1897 & & \\
\hline$» \quad »$ Junior & $》$ & 1854 & & \\
\hline "Rodrigues de Azevedo. & » & 1891 & & \\
\hline Landulpho da Rocha Medrado. & Bahia & 1887 & & \\
\hline Lyra da Silva. & Rio de Janeiro & 1852 & & \\
\hline Machado Nunes & $\gg$ & 1836 & & \\
\hline Possolo & Côrte & 1887 & & \\
\hline Ribeiro de Almeida & Rio G. do Sul & 1835 & & \\
\hline Rodrigues Barcellos. & $\gg$ & 1861 & & \\
\hline Vicente Ribas da Silva & Paraná & 1895 & & \\
\hline Serafim dos Anjos França. & Rio G. do Sul & 1836 & & \\
\hline » Muniz Barreto & Babia & 1856 & & \\
\hline Sergio Francisco de Souza Castro. & S. Paulo & 1861 & & \\
\hline$\gg \gg \gg \gg$ Junior & Paraná & 1894 & & \\
\hline » Lopes Falcão. . & Santa Catharina & 1844 & & \\
\hline »Ulrich de Macedo & Rio G. do Sul & 1894 & & \\
\hline Severiano Emilio de Figueiredo & Bahia & 1900 & & \\
\hline Severino Alves de Carvalho. & Maranhão & 1852 & & \\
\hline$» \quad$ de Freitas Prestes & Rio G. do Sul & 1879 & & \\
\hline dos Santos Vieira. & BaLia & 1874 & & \\
\hline Eulogo Ribeiro de Rezende. & Minas Geraes & 1867 & & \\
\hline Severo Mendes dos Santos Ribeiro & $»$ & 1867 & & \\
\hline » Peixoto de Oliveira Barcellos. & Rio G. do 'Sul & 1883 & & \\
\hline Silverio Fernandes de Araujo Jorge & Alagôas & 1840 & & \\
\hline » Gonzaga de Carvalho Amorim & Rio de Janeiro & 1867 & & \\
\hline Silvino Braulio Cesar & S. Paulo & 1886 & & \\
\hline » Cavalcanti d'Albuquerque & Pernambuco & 1852 & & \\
\hline$» \quad$ Soares de Mello. & Rio de Janeiro & 1863 & & \\
\hline Silvio Tibiriçá de Almeida & Minas Geraes & 1892 & & \\
\hline Simão Eugenio de Oliveira Lima & S. Paulo & 1881 & & \\
\hline Simpliciano da Rocha Pombo & $»$ & 1863 & & \\
\hline » de Souza Lima. & Minas Geraes & 1867 & & \\
\hline Sizenando Barreto Nabuco de Araujo. & Pernambuco & 1864 & & \\
\hline
\end{tabular}




\begin{tabular}{|c|c|c|c|c|}
\hline \multirow[b]{2}{*}{ rI } & \multirow[b]{2}{*}{ NATURALIDADE } & \multicolumn{3}{|c|}{ SCIENCIAS } \\
\hline & & 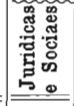 & 萢 & 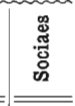 \\
\hline Symphorozo de Lara Fernandes & Rio de Janeiro & 1890 & & \\
\hline Tancredo Pitta Pinheiro & Rio G. do Sul & 1890 & & \\
\hline Tertuliano Moreira Cesar & S. Paulo & 1879 & & \\
\hline$\gg \quad$ Teixeira de Freitas & Bahia & 1859 & & \\
\hline Theodomiro Alves Pereira & Minas Geraes & 1863 & & \\
\hline$» \quad$ de Araujo Cintra & S. Paulo & 1896 & & \\
\hline Theodoro de Barros Machado da Silva & Capital Federal & 1891 & & \\
\hline$\gg \quad »$ Macedo Sodré & Rio de Janeiro & 1868 & & \\
\hline » Dias de Carvalho Junior & Minas Geraes & 1883 & & \\
\hline Reichert Junior & S. Paulo & 1883 & & \\
\hline Torquato Pinto e Silva. & $\gg$ & 1886 & & \\
\hline Theodosio Manoel Soares de Souza & Minas Geraes & 1835 & & \\
\hline Theophilo Beredicto de Souza Carvalho & S. Paulo & 1892 & & \\
\hline » Carlos Benedicto Ottoni & Rio de Janeiro & 1863 & & \\
\hline de Almeida Fortuna & Ceará & - & 1898 & 1896 \\
\hline Dias de Mesquita & Maranhão & 1881 & & \\
\hline José Antunes Braga. & S. Paulo & 1874 & & \\
\hline Nobrega & $»$ & 1897 & & \\
\hline$» \quad$ Ayroza & Minas Geraes & 1858 & & \\
\hline Pereira da Silva. & $»$ & 1865 & & \\
\hline Ribeiro de Andrade & $»$ & 1894 & & \\
\hline$» \quad$ Rezende Junior & S. Paulo & 1871 & & \\
\hline Tavares Paes & Minas Geraes & 1859 & & \\
\hline Victorio Ribeiro de Rezende & S. Paulo & 1837 & & \\
\hline Theotonio de Miranda Lima & Minas Geraes & 1868 & & \\
\hline » Fernandes da Costa Pereira & Rio de Janeiro & 1868 & & \\
\hline Thimoteo Pereira da Rosa (I. $\left.{ }^{\circ}\right)$ & Rio G. do Sul & 1859 & & \\
\hline$\gg \gg \gg \gg\left(2 .^{\circ}\right)$ & & 1890 & & \\
\hline Thomaz Alves Junior . & Rio de Janeiro & 1854 & & \\
\hline » Augusto Ribeiro de Lima & S. Paulo & 1886 & & \\
\hline$» \quad$ d'Aquino Leite & Minas Geraes & 1862 & & \\
\hline da Silva Brandão & $"$ & 1888 & & \\
\hline Dias Leite. & S. Paulo & 1888 & & \\
\hline Eurico Gomes. & Bahia & 1884 & & \\
\hline Fortunato de Brito & Rio de Janeiro & 1841 & & \\
\hline José Coelho de Almeida. & $\gg$ & 1861 & & \\
\hline Malheiros & Rio G. do Sul & - & 1897 & 71896 \\
\hline Pimentel & S. Paulo & - & 1895 & \\
\hline Wallace da Gama Cochrane & , & 1883 & & \\
\hline Thomé Pires d'Avila Netto. & & 1870 & & \\
\hline Tiburcio Valeriano da Rocha Lins & Alagôas & 1860 & & \\
\hline Tito Augusto de Toledo Blak & S. Paulo & 1892 & & \\
\hline$\gg \quad » \quad$ Pereira de Mattos & Bahia & 1857 & & \\
\hline » de Souza Rodrigues & S. Paulo & 1884 & & \\
\hline " Fulgencio Alves Pereira & Minas Geraes & 1884 & & \\
\hline » Livio Vieira Dórtas & Sergipe & 1866 & & \\
\hline »Prates da Silva & Rio G. do Sul & 1877 & & \\
\hline
\end{tabular}




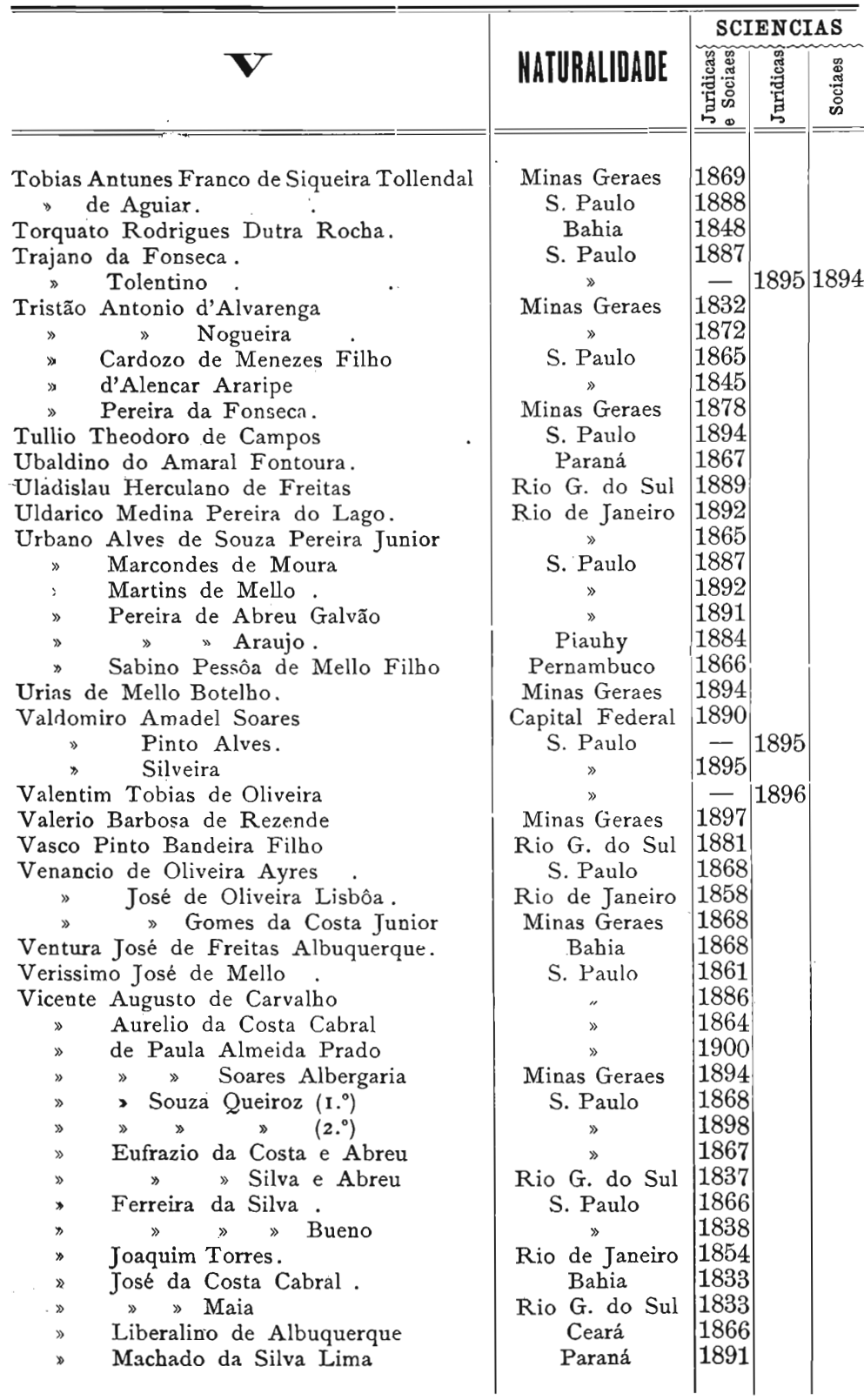




\begin{tabular}{|c|c|c|c|c|}
\hline \multirow{2}{*}{2} & \multirow[b]{2}{*}{ YATURALIDADE } & \multicolumn{3}{|c|}{ SCIENCIAS } \\
\hline & & 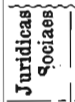 & 氶 & 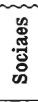 \\
\hline Vicente Mamede de Freitas & S. Paulo & 1855 & & \\
\hline » Miguel da Silva Abreu & Goyaz & 1893 & & \\
\hline Pires da Motta (P.॰) & S. Paulo & 1832 & & \\
\hline Xavier de Toledo Sobrinho & Minas Geraes & 1868 & & \\
\hline Victor Manoel de Souza Lima. & Rio de Janeiro & 1882 & & \\
\hline$\gg \gg \gg$ Monteiro. & Côrte & 1881 & & \\
\hline » Marques da Silva Ayrosa & S. Paulo & 1887 & & \\
\hline Victorino Antonio do Sacramento & Bahia & 1867 & & \\
\hline , Caetano de Brito. & S. Paulo & 1861 & & \\
\hline » José Carneiro Monteiro & Rio G. do Sul & 1883 & & \\
\hline Vindilino Furtado de Mendonça & Minas Geraes & 1892 & & \\
\hline Virgilio Augusto de Araujo & S. Paulo & - & 1895 & \\
\hline » de Toledo Malta & $»$ & 1884 & & \\
\hline » $\quad$ Siqueira Cardozo & $\gg$ & 1857 & & \\
\hline Francisco Caldas . & 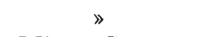 & 1891 & & \\
\hline Martins de Mello Franco. & Minas Geraes & 1866 & & \\
\hline Moretz-Sohn & $\gg$ & 1881 & & \\
\hline Ramos frordilho & Bahia & 1881 & & \\
\hline$» \quad$ Vieira . : & S. Paulo & 1893 & & \\
\hline Virginio Amancio Pereira Leite & Rio de Janeiro & 1866 & & \\
\hline Viriato Diniz Mascarenhas. & Minas Geraes & 1887 & & \\
\hline Waldomiro Guilherme Christiano & $\gg$ & 1883 & & \\
\hline Wenceslau Braz Pereira Gomes & $»$ & 1890 & & \\
\hline$» \quad$ José de Oliveira Queiroz & S. Paulo & 1890 & & \\
\hline Pereira de Escobar. & Rio G. do Sul & 1880 & & \\
\hline Whashington Badaró . . & Minas Geraes & 1884 & & \\
\hline$» \quad$ Luiz Pereira de Souza & Rio de Janeiro & 1891 & & \\
\hline Osorio de Oliveira & $\gg$ & - & 1898 & \\
\hline Rodrigues Pereira & Minas Geraes & 1883 & & \\
\hline Wladimir do Nascimento Matta & S. Paulo & 1889 & & \\
\hline Wladimiro Augusto do Amaral & $»$ & 1891 & & \\
\hline Zeferino de Faria Filho & Côrte & 1882 & & \\
\hline Zoroastro Augusto Pamplona . & Pernambuco & 1862 & & \\
\hline
\end{tabular}

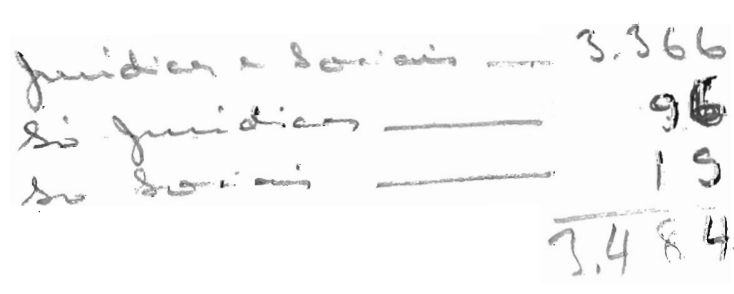




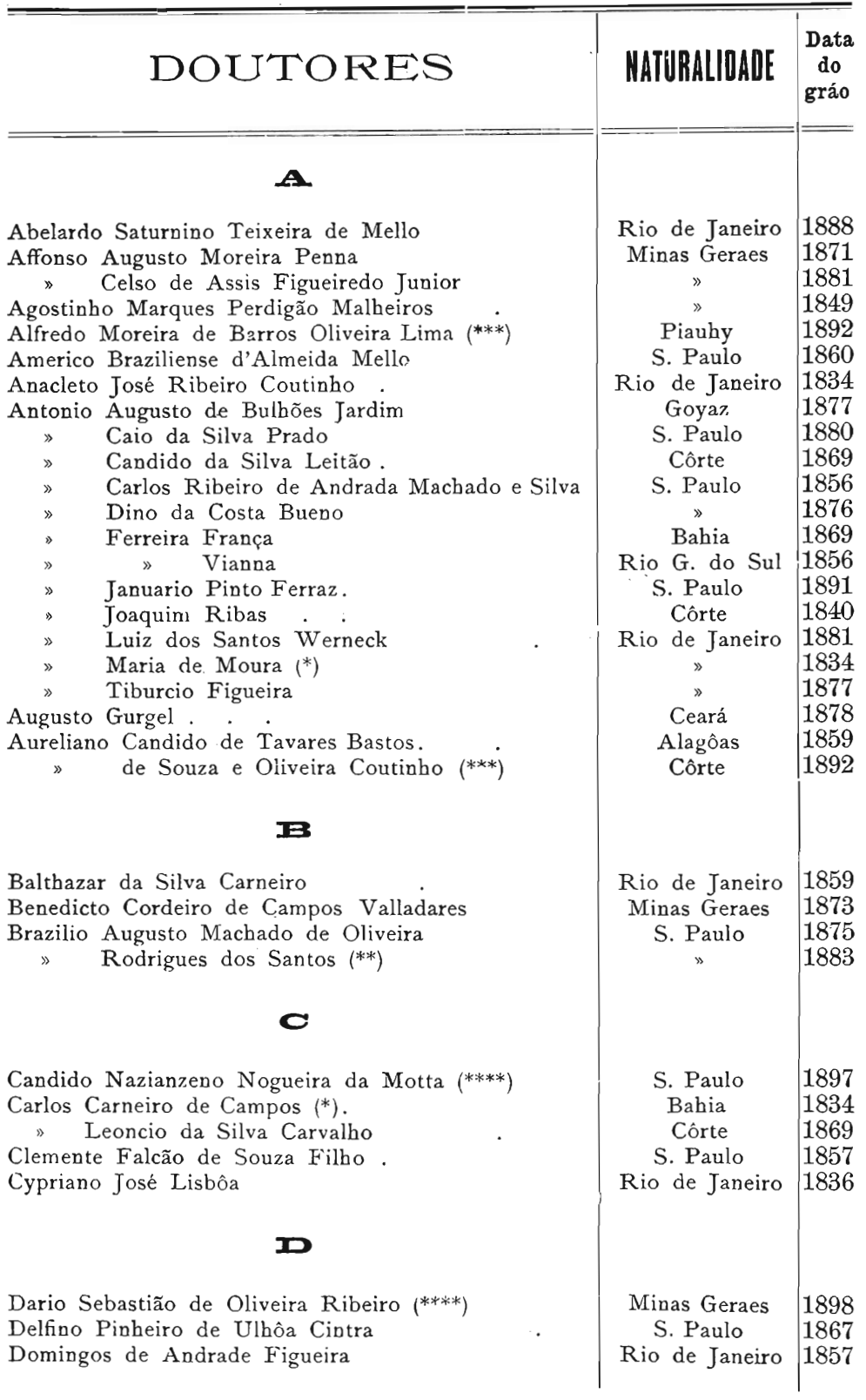




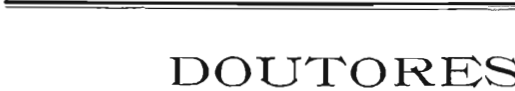

IE

Eduardo Olympio Machado.

Egydio Barbosa de Oliveira Itaqui

Emilio Valentim Barrios

Emygdio Joaquim dos Santos

Ernesto Ferreira França.

$$
\text { Moura (***). }
$$

Ezequiel de Paula Ramos

\section{Eా}

Fernando Mendes de Almeida.

Francisco Antonio de Araujo

\begin{tabular}{|c|c|}
\hline$》$ & Dutra Rodrigues \\
\hline$\gg$ & Bernardino Ribeiro. \\
\hline » & de Assis do Monte Carmello \\
\hline$»$ & Gomes dos Santos Lopes \\
\hline$》$ & José Ferreira Baptista \\
\hline$»$ & Justino Gonçalves de Andrade \\
\hline 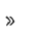 & Maria de Souza Furtado de Mendonça \\
\hline & "Velho da Veiga \\
\hline
\end{tabular}

\section{G}

Gabriel José Rodrigues de Rezende (****)

" $"$ dos Santos

Genuino Firmino Vidal Capistrano

\section{I포}

Hygino Alves de Abreu e Silva

\section{I}

Ildefonso Xavier Ferreira

Jesuino Ubaldo Cardoso de Mello.

João Baptista Pereira

» Carlos de Oliveira Maia

*. Chrispiniano Soares

Babia

1846

Rio G. do Sul 1863

Babia

1862

Pernamibuco

1866

S. Paulo

1860

$\gg$

1893

1867

Maranhão

Bahia

Rio de Janeiro

>

S. Paulo

Rio de Janeiro

Portugal

Loanda

Rio de Janeiro

S. Paulo

1880

1834

1866

1835

1836

1860

1834

1851

1839

(

1849

Minas Geraes

S. Paulo

Santa Catharina

1874

Mínas Geraes

S. Paulo

S. Paulo

1887

Côrte

1858

S. Paulo

1859

»

1835 


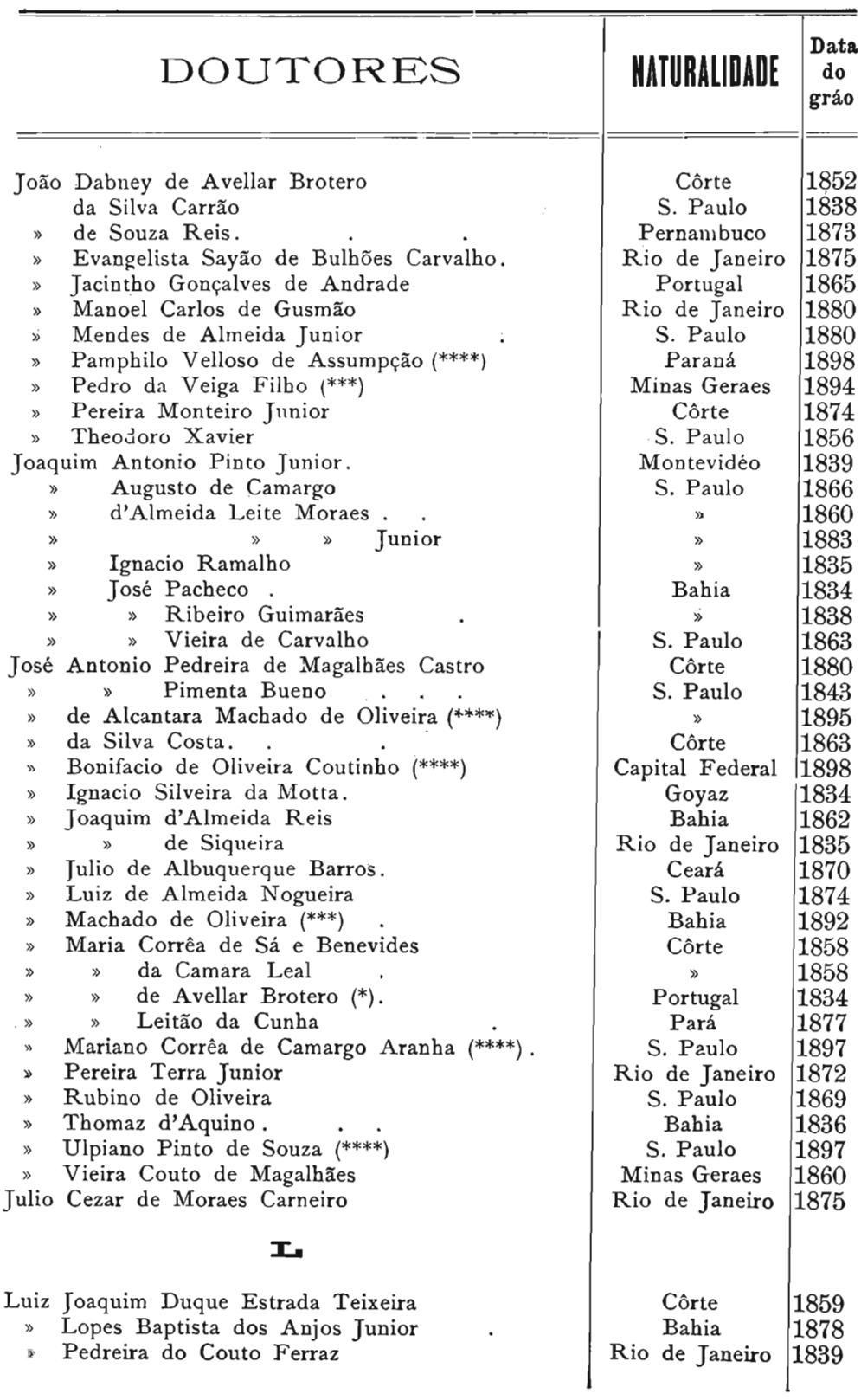




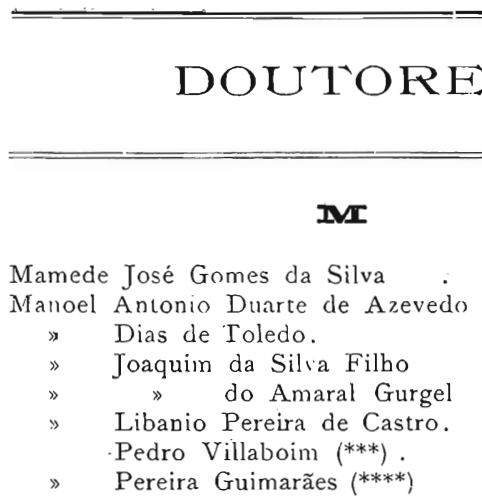

Marcellino José da Ribeira Silva Bueno

Martim Francisco Ribeiro de Andrada

Miguel Archanjo Ribeiro de Castro Camargo

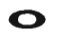

Olegario Herculano d'Aquino e Castro

Osorio Dias de Aguiar Souza (****)

$$
\mathbf{E}
$$

Paulo Antonio do Valle

Pedro Augusto Carneiro Lessa.

»Elias Martins Pereira

"Vicente de Azevedo.

Prudencio Geraldes Tavares da Veiga Cabral (*).

\section{ER}

Raphael Corrêa da Silva Sobrinho (****)

$$
\text { » de Araujo Ribeiro }
$$

Reynaldo Porchat (****).

Rodrigo Octavio de Oliveira Menezes.

Romualdo de Andrade Baena

\section{S}

Severino de Freitas Prestes.

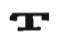

Tito Augusto Pereira de Mattos

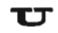

Uladislau Herculano de Frcitas $\left(^{* * *}\right)$
S. Paulo 1858

Rio de Janeiro 1859

S. Paulo 1833

Côrte 1880

S. Paulo 1833

Babia 1834

S. Paulo 1897

» 1834

França $\quad 1856$

S. Paulo 1834

S. Paulo 1849

» 1899

S. Paulo

1860

Minas Geraes 1888

S. Paulo 1860

Matto Grosso 1834

S. Paulo 1895

Rin G. do Sul 1834

S. Paulo 1897

S. Paulo 1862

Rio de Janeiro 1875

Rio G. do Sul 1880

Bahia

1858

Rio G. do Sul 1892 


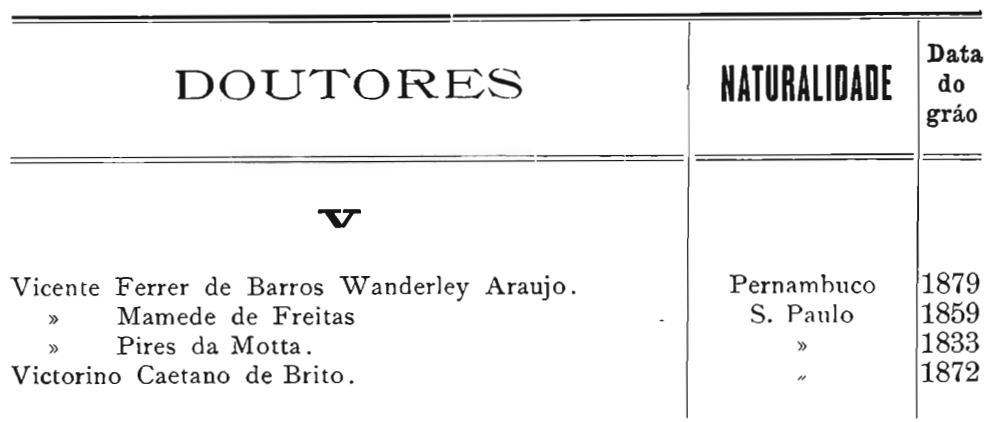

(“) Foi conferido o gráo em virtude do Decreto $\mathrm{n} .{ }^{\circ} 34$ de 16 de S.tembro de 1834

(“) Foi conferido o gráo cm vista do Aviso do Ministerio dos Nogocios do Imperio de 25 de Setembro de 1883, e do disposto no art. 49 dos Estatutos approvados pelo Decreto n.' 1386 de 28 de Abril de 1854.

(“*) Foi conferido o gráo en virtude do disposto no art. 112 do Decreto n. $1232 \mathrm{~F}-$ de 2 de Janeiro de 1891 e do art. 83 do Codigo do Ensino approvado pelo Decreto n. 1159 de 3 de Dezembro de 1892.

(“...) Foi conferido o gráo em virtude do art. 112 do Decreto n. 1232 F-de 2 de Janeiro de 1891 e do art. 83 do Codigo do Ensino approvado pelo Decreto n. ${ }^{\circ} 1159$ de 3 de Dezerabro de 1892 e pelo Decreto Legislativo n. 230 -de 7 de Dezembro de 1894. 


\section{DIRECTORES}

Tenente-General José de Arouche de Toledo Rendon

Dr. Carlos Carneiro de Campos

Dr. José da Costa Carvalho

Senador Luiz Nicoláu de Campos Vergueiro.

Dr. Manoel Joaquim do Amaral Gurgel

Conselheiro Dr. Vicente Pires da Motta

Conselheiro Dr. Carlos Leoncio de Carvalho.

\section{VICE-DIRECTOR}

Dr. João Pereira Monteiro

\section{LENTES CATHEDRATICOS}

Dr. José Maria de Avellar Brotero

Dr. Balthazar da Silva Lisbôa.

Dr. Antonio Maria de Moura

Dr. Luiz Nicoláu Fagundes Varella

Dr. Carlos Carneiro de Campos

Dr. José Joaquim Fernandes Torres

Dr. Prudencio Geraldes Tavares da Veiga Cabral

Dr. Thomaz José Finto de Cerqueira.

Dr. José Candido de Deus e Silva

Dr. Clemente Falcão de Souza.

Dr. Manoel Joaquim do Amaral Gurgel

1830

1831

Dr. Vicente Pires da Motta

Dr. Manoel Dias de Toledo

Dr. Anacleto José Ribeiro Coutinho

Dr. José Ignacio Silveira da Motta

Dr. João Chrispiniano Soares

Dr. Joaquim Ignacio Ramalho

Dr. Francisco Maria de Souza Furtado de Mendonça

Dr. Luiz Pedreira do Couto Ferraz

Dr. João da Silva Carrão

Dr. Martim Francisco Ribeiro de Andrada 1859

Dr. Antonio Joaquim Ribas

Dr. José Bonifacio de Andrada e Silva

Dr. Antonio Carlos Ribeiro de Andrada Machado e Silva. 
Dr. Francisco Justino Gonçalves de Andrade.

Dr. Clemente Falcão de Souza Filho

Dr. João Theodoro Xavier.

Dr. Ernesto Ferreira França

Dr. Manoel Antonio Duarte de Azevedo. .

Dr. José Maria Corrêa de Sá e Benevides

Dr. João Jacintho Gonçalves de Andrade.

Dr. Cárlos Leoncio de Carvalbo

1878

1881

Dr. Francisco Antonio Dutra Rodrigues

1881

Dr. Joaquim José Vieira de Carvalho.

Dr. Joaquim Augusto de Camargo.

1881

1881

Dr. Joaquim de Almeida Leite Moraes

1882

Dr. José Rubino de Oliveira

Dr. João Pereira Monteiro

1882

1883

Dr. Vicente Mamede de Freitas

1887

Dr. Americo Braziliense de Almeida Mello

1888

Dr. Antonio Dino da Costa Bueno.

1890

Dr. Brasilio Augusto Machado de Oliveira

1890

Dr. Brasilio Rodrigues dos Santos

1891

Dr. Frederico José Cardoso de Araujo Abranches

1891

Dr. Pedro Augusto Carneiro Lessa.

1891

Dr. Manoel Clementino de Oliveira Escorel

1891

Dr. João Mendes de Almeida Junior

Dr. José Luiz de Almeida Nogueira

1891

Dr. Antonio Amancio Pereira de Carvalbo

1890

1891

Dr. Uladislau Herculano de Freitas

1891

Dr. Jesuino Ubaldo Cardoso de Mello

1891

Dr. Antonio de Campos Toledo

1891

Dr. Antonio Januario Pinto Ferraz

1891

Dr. Aureliano de Souza e Oliveira Coutinho.

1891

Dr. Augusto Cesar de Miranda Azevedo

1891

Dr. Ernesto Moura

1891

Dr. Alfredo Moreira de Barros Oliveira Lima

1895

Dr. Manoel Pedro Villaboim

Dr. José Macbado de Oliveira

1892

1895

Dr. Severino de Freitas Prestes

1895

\begin{tabular}{l|l} 
Dr. João Pedro da Veiga Filho $\quad$. & 1897
\end{tabular}

\section{LENTES SUBSTITUTOS}

Dr. Francisco José Ferreira Baptista

Dr. Francisco Bernardino Ribeiro

Dr. Gabriel José Rodrigues dos Santos

Dr. João Dabney de Avellar Brotero

Dr. João José de Almeida Reis.

Dr. José Xavier Carvalho de Mendonça

1856

1871

Dr. Augusto Miranda

Dr. Raphael Corrêa da Silva Sobrinho 
Dr. José de Alcantara Machado de Oliveira

Dr. José Ulpiano Pinto de Souza.

Dr. Candido Nazianzeno Nogueira da Motta.

1897

Dr. José Mariano Corrêa de Camargo Aranha

1897

Dr. Gabriel José Rodrigues de Rezende

1897

Dr. Reynaldo Porchat

1897

Dr. Dario Sebastião de Oliveira Ribeiro

1898

Secretaria da Faculdade de Direito de São Paulo, 3 I dé Dezembro de 1900.

O Subsecretario,

Grutio Goaquim Gonfalves Maia 